\title{
$\beta$-Hydride Elimination at Low-Coordinate Gold(III) Centers
}

Feriel Rekhroukh, Laura Estevez, Sonia Mallet-Ladeira, Karinne Miqueu,* Abderrahmane Amgoune, * Didier Bourissou *

\section{Supporting Information}

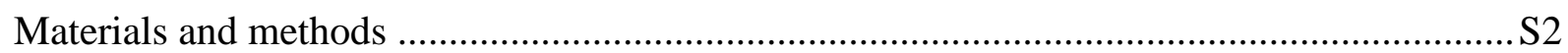

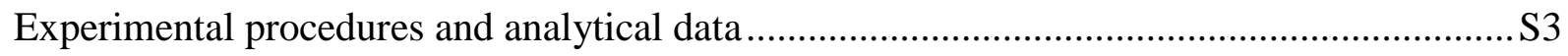

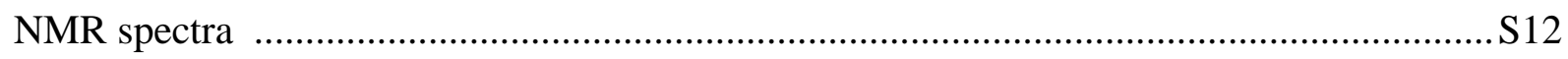

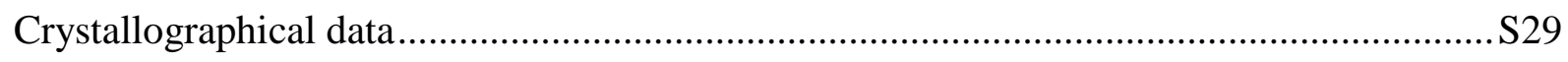

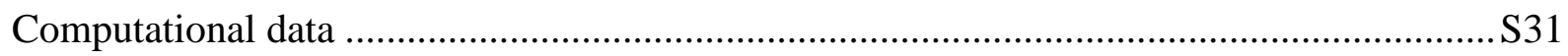




\section{Materials and methods}

All reactions and manipulations were carried out under an atmosphere of dry argon using standard Schlenk techniques or in a glovebox under inert atmosphere. Dry, oxygen-free solvents were employed. Melting points were determined with a Stuart SMP40 apparatus and are uncorrected. Solution ${ }^{1} \mathrm{H},{ }^{13} \mathrm{C},{ }^{11} \mathrm{~B},{ }^{19} \mathrm{~F}$ and ${ }^{31} \mathrm{P}$ NMR spectra were recorded on Bruker Avance 300, 400 or 500 spectrometers, at $298 \mathrm{~K}$ unless otherwise specified. Chemical shifts $(\delta)$ are expressed with a positive sign, in parts per million. ${ }^{1} \mathrm{H}$ and ${ }^{13} \mathrm{C}$ chemical shifts reported are referenced internally to residual protio- $\left({ }^{1} \mathrm{H}\right)$ or deutero- $\left({ }^{13} \mathrm{C}\right)$ solvent, while ${ }^{31} \mathrm{P}$ chemical shifts are relative to $85 \% \mathrm{H}_{3} \mathrm{PO}_{4},{ }^{11} \mathrm{~B}$ chemical shifts are relative to $\mathrm{BF}_{3} \cdot \mathrm{OEt}_{2}$ and ${ }^{19} \mathrm{~F}_{\text {chemical shifts }}$ are relative to $\mathrm{CFCl}_{3}$ as external reference. The following abbreviations and their combinations are used: br, broad; s, singlet; d, doublet; t, triplet; q, quartet, m, multiplet. The ${ }^{1} \mathrm{H}$ and ${ }^{13} \mathrm{C}$ resonance signals were attributed by means of 2D HSQC and HMBC experiments. Mass spectra were recorded on a Waters LCT apparatus. GC/MS was performed on a Perkin Elmer Clarus 500 machine equipped with a head-space injector with a BPX-volatiles column. All starting materials were purchased from Aldrich. Complex 1 was prepared following previously described procedure ${ }^{1}$.

\footnotetext{
${ }^{1}$ Rekhroukh, F.; Brousses, R.; Amgoune, A.; Bourissou, D. Angew. Chem. Int. Ed. 2015, 54, 1266.
} 


\section{Experimental procedures and analytical data}

\section{General procedure for the synthesis of the (P,C) gold(III) alkyl complexes 2a-c.}

A solution of $\operatorname{RMgX}$ (1.2 equiv, 2M in diethyl ether) was added dropwise to a solution of the gold(III) di-iodo complex 1 (250 mg, $0.36 \mathrm{mmol})$ in $\mathrm{THF}(15 \mathrm{~mL})$ at $-78^{\circ} \mathrm{C}$. The reaction mixture was stirred for $1 \mathrm{~h}$ at $-78^{\circ} \mathrm{C}$ and then at room temperature for $1 \mathrm{~h}$. While warming to room temperature, the reaction mixture cleared up to light yellow. The solvent was then removed under vacuum. The residue was dissolved in DCM $(8 \mathrm{~mL})$ and washed with distilled water $(1 \mathrm{~mL})$. The organic phase was dried over $\mathrm{Na}_{2} \mathrm{SO}_{4}$ and filtered through celite, all volatiles were then removed in vacuum to yield the product as a yellow solid. Complexes were purified by flash column chromatography (silica, eluent: Pentane/DCM 80:20).

\section{(P,C) Gold iodo $n$-propyl complex 2a}

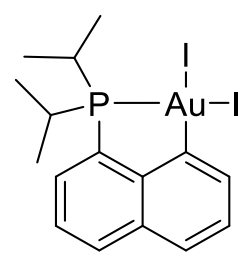

1

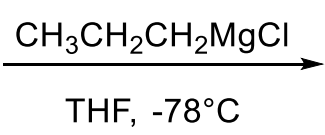

THF, $-78^{\circ} \mathrm{C}$

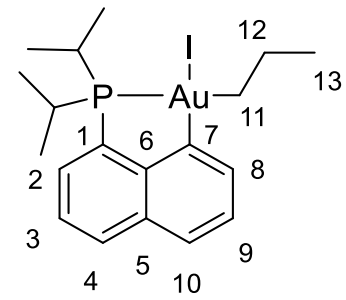

2a

Yield: $69 \%$.

Mp: $88.5^{\circ} \mathrm{C} ;{ }^{1} \mathbf{H}$ NMR $\left(300 \mathrm{MHz}, \mathrm{CD}_{2} \mathrm{Cl}_{2}\right): \delta 8.03\left(\mathrm{~m}, 1 \mathrm{H}, \mathrm{H}_{4}\right), 7.94(\mathrm{~m}, 1 \mathrm{H}, \mathrm{H}$ ) $), 7.77-7.88$ $\left(\mathrm{m}, 2 \mathrm{H}, \mathrm{H}_{3} \& \mathrm{H}_{10}\right), 7.55-7.63\left(\mathrm{~m}, 2 \mathrm{H}, \mathrm{H}_{2} \& \mathrm{H}_{8}\right), 3.14$ (heptd, ${ }^{3} J_{\mathrm{HH}}=7.1 \mathrm{~Hz},{ }^{2} J_{\mathrm{PH}}=11.5 \mathrm{~Hz}, 2 \mathrm{H}$, $\mathrm{CH} i \mathrm{Pr}), 2.39\left(\mathrm{~m}, 2 \mathrm{H}, \mathrm{H}_{11}\right), 1.99\left(\mathrm{~m}, 2 \mathrm{H}, \mathrm{H}_{12}\right), 1.30\left(\mathrm{dd},{ }^{3} J_{\mathrm{HH}}=7.1 \mathrm{~Hz},{ }^{3} J_{\mathrm{PH}}=17.8 \mathrm{~Hz}, 6 \mathrm{H}\right.$, $\left.\mathrm{CH}_{3} i \mathrm{Pr}\right), 1.18\left(\mathrm{dd},{ }^{3} J_{\mathrm{HH}}=7.0 \mathrm{~Hz},{ }^{3} J_{\mathrm{PH}}=15.6 \mathrm{~Hz}, 6 \mathrm{H}, \mathrm{CH}_{3} i \mathrm{Pr}\right), 1.11\left(\mathrm{td},{ }^{3} J_{\mathrm{HH}}=7.2 \mathrm{~Hz}, J_{\mathrm{PH}}=\right.$ $\left.1.2 \mathrm{~Hz}, 3 \mathrm{H}, \mathrm{H}_{13}\right) ;{ }^{31} \mathbf{P}\left\{{ }^{1} \mathbf{H}\right\}$ NMR $\left(121 \mathrm{MHz}, \mathrm{CD}_{2} \mathrm{Cl}_{2}\right): \delta 68.4(\mathrm{~s}) ;{ }^{13} \mathbf{C}\left\{{ }^{1} \mathbf{H}\right\}$ NMR $(75 \mathrm{MHz}$, $\left.\mathrm{CD}_{2} \mathrm{Cl}_{2}\right): \delta 150.0\left(\mathrm{~d},{ }^{3} J_{\mathrm{PC}}=3.1 \mathrm{~Hz}, \mathrm{C}_{7}\right), 149.8\left(\mathrm{~s}, \mathrm{C}_{6}\right), 135.3\left(\mathrm{~d},{ }^{3} J_{\mathrm{PC}}=13.6 \mathrm{~Hz}, \mathrm{C}_{5}\right), 133.1(\mathrm{~d}$, $\left.{ }^{4} J_{\mathrm{PC}}=2.2 \mathrm{~Hz}, \mathrm{C}_{4}\right), 131.4\left(\mathrm{~s}, \mathrm{C}_{3}\right), 130.0\left(\mathrm{~d},{ }^{1} J_{\mathrm{PC}}=44.1 \mathrm{~Hz}, \mathrm{C}_{1}\right), 128.9\left(\mathrm{~s}, \mathrm{C}_{9}\right), 127.7\left(\mathrm{~s}, \mathrm{C}_{10}\right)$, $127.5\left(\mathrm{~s}, \mathrm{C}_{8}\right), 126.2\left(\mathrm{~d},{ }^{2} J_{\mathrm{PC}}=7.0 \mathrm{~Hz}, \mathrm{C}_{2}\right), 49.7\left(\mathrm{~d},{ }^{2} J_{\mathrm{PC}}=102.7 \mathrm{~Hz}, \mathrm{C}_{11}\right), 24.8\left(\mathrm{~d},{ }^{3} J_{\mathrm{PC}}=5.4 \mathrm{~Hz}\right.$, $\left.\mathrm{C}_{12}\right), 27.1\left(\mathrm{~d},{ }^{1} J_{\mathrm{PC}}=22.8 \mathrm{~Hz}, \mathrm{CH} i P r\right), 19.1\left(\mathrm{~d},{ }^{4} J_{\mathrm{PC}}=13.3 \mathrm{~Hz}, \mathrm{C}_{13}\right), 18.9\left(\mathrm{~d},{ }^{2} J_{\mathrm{PC}}=3.2 \mathrm{~Hz}\right.$, $\mathrm{CH}_{3} i \mathrm{Pr}$ ), $18.5\left(\mathrm{~d},{ }^{2} J_{\mathrm{PC}}=1.2 \mathrm{~Hz}, \mathrm{CH}_{3} i \mathrm{Pr}\right.$ ); HRMS (ESI+): calcd for $[\mathrm{M}]^{+}=\mathrm{C}_{19} \mathrm{H}_{27} \mathrm{AuP}^{+}: 483.1516$. Found: 483.1522. 


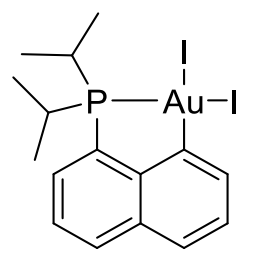

1

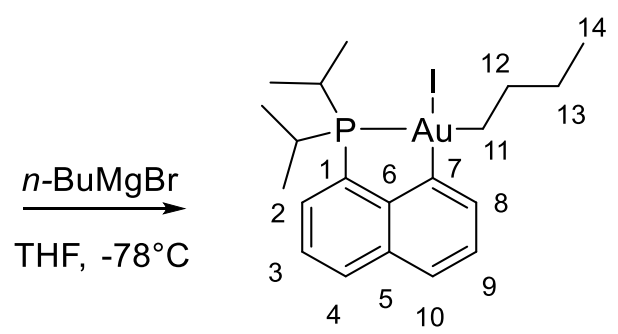

$2 \mathbf{b}$

Yield: $66 \%$.

Mp: $69.3{ }^{\circ} \mathrm{C} ;{ }^{1} \mathbf{H}$ NMR $\left(500 \mathrm{MHz}, \mathrm{CD}_{2} \mathrm{Cl}_{2}\right): \delta 8.03\left(\mathrm{dt},{ }^{3} \mathrm{~J}_{\mathrm{HH}}=7.0 \mathrm{~Hz},{ }^{5} J_{\mathrm{PH}}=1.6 \mathrm{~Hz}, 1 \mathrm{H}, \mathrm{H}_{4}\right)$, $7.93(\mathrm{bm}, 1 \mathrm{H}, \mathrm{H} 9), 7.85\left(\mathrm{bm}, 1 \mathrm{H}, \mathrm{H}_{10}\right), 7.79\left(\mathrm{ddt},{ }^{3} J_{\mathrm{HH}}=7.0 \mathrm{~Hz},{ }^{3} J_{\mathrm{HH}}=1.1 \mathrm{~Hz},{ }^{4} J_{\mathrm{PH}}=7.2 \mathrm{~Hz}\right.$, $\left.1 \mathrm{H}, \mathrm{H}_{3}\right), 7.56-7.62\left(\mathrm{~m}, 2 \mathrm{H}, \mathrm{H}_{2} \& \mathrm{H}_{8}\right), 3.09-3.19(\mathrm{~m}, 2 \mathrm{H}, \mathrm{CH} i \mathrm{Pr}), 2.40\left(\mathrm{td},{ }^{3} J_{\mathrm{HH}}=7.7 \mathrm{~Hz},{ }^{3} J_{\mathrm{PH}}=\right.$ $\left.7.6 \mathrm{~Hz}, 2 \mathrm{H}, \mathrm{H}_{11}\right), 1.92\left(\mathrm{~m}, 2 \mathrm{H}, \mathrm{H}_{12}\right), 1.51\left(\mathrm{sex},{ }^{3} J_{\mathrm{HH}}=7.4 \mathrm{~Hz}, 2 \mathrm{H}, \mathrm{H}_{13}\right), 1.30\left(\mathrm{dd},{ }^{3} J_{\mathrm{HH}}=7.1 \mathrm{~Hz}\right.$, $\left.{ }^{3} J_{\mathrm{PH}}=15.6 \mathrm{~Hz}, 6 \mathrm{H}, \mathrm{CH}_{3} i \mathrm{Pr}\right), 1.18\left(\mathrm{dd},{ }^{3} J_{\mathrm{HH}}=7.1 \mathrm{~Hz},{ }^{3} J_{\mathrm{PH}}=15.6 \mathrm{~Hz}, 6 \mathrm{H}, \mathrm{CH}_{3} i \mathrm{Pr}\right), 0.97\left(\mathrm{t},{ }^{3} J_{\mathrm{HH}}\right.$ $\left.=7.4 \mathrm{~Hz}, 3 \mathrm{H}, \mathrm{H}_{14}\right) ;{ }^{31} \mathbf{P}\left\{{ }^{1} \mathbf{H}\right\}$ NMR $\left(202 \mathrm{MHz}, \mathrm{CD}_{2} \mathrm{Cl}_{2}\right): \delta 68.4(\mathrm{~s}) ;{ }^{13} \mathbf{C}\left\{{ }^{1} \mathbf{H}\right\} \mathbf{N M R}(125 \mathrm{MHz}$, $\left.\mathrm{CD}_{2} \mathrm{Cl}_{2}\right): \delta 150.0\left(\mathrm{~d},{ }^{3} J_{\mathrm{PC}}=3.1 \mathrm{~Hz}, \mathrm{C}_{7}\right), 149.6\left(\mathrm{~d},{ }^{2} J_{\mathrm{PC}}=33.1 \mathrm{~Hz}, \mathrm{C}_{6}\right), 135.3\left(\mathrm{~d},{ }^{3} J_{\mathrm{PC}}=13.7 \mathrm{~Hz}\right.$, $\left.\mathrm{C}_{5}\right), 133.0\left(\mathrm{~d},{ }^{4} J_{\mathrm{PC}}=2.2 \mathrm{~Hz}, \mathrm{C}_{4}\right), 131.4\left(\mathrm{~s}, \mathrm{C}_{3}\right), 130.0\left(\mathrm{~d},{ }^{1} J_{\mathrm{PC}}=44.0 \mathrm{~Hz}, \mathrm{C}_{1}\right), 128.9\left(\mathrm{~s}, \mathrm{C}_{9}\right)$, $127.7\left(\mathrm{~s}, \mathrm{C}_{10}\right), 127.6\left(\mathrm{~s}, \mathrm{C}_{8}\right), 126.2\left(\mathrm{~d},{ }^{2} J_{\mathrm{PC}}=7.1 \mathrm{~Hz}, \mathrm{C}_{2}\right), 47.3\left(\mathrm{~d},{ }^{2} J_{\mathrm{PC}}=102.9 \mathrm{~Hz}, \mathrm{C}_{11}\right), 33.7$ $\left(\mathrm{d},{ }^{3} J_{\mathrm{PC}}=5.4 \mathrm{~Hz}, \mathrm{C}_{12}\right), 27.8\left(\mathrm{~d},{ }^{4} J_{\mathrm{PC}}=13.1 \mathrm{~Hz}, \mathrm{C}_{13}\right), 27.1\left(\mathrm{~d},{ }^{1} J_{\mathrm{PC}}=22.8 \mathrm{~Hz}, \mathrm{CHiPr}\right), 19.0(\mathrm{~d}$, $\left.{ }^{2} J_{\mathrm{PC}}=3.2 \mathrm{~Hz}, \mathrm{CH}_{3} i P r\right), 18.5$ (s, $\left.\mathrm{CH}_{3} i P r\right), 14.5$ (s, C 14 ); HRMS (ESI+): calcd for $\left[\mathrm{M}^{+}=\right.$ $\mathrm{C}_{20} \mathrm{H}_{29} \mathrm{AuP}^{+}$: 497.1672. Found: 497.1678. 


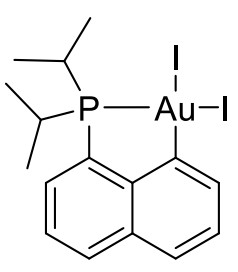

1

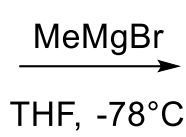

$\mathrm{THF},-78^{\circ} \mathrm{C}$

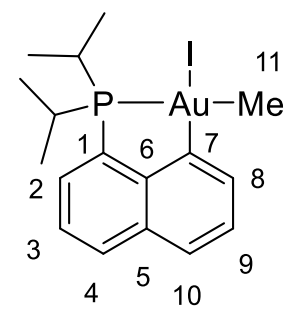

2c

Yield: $70 \%$.

Mp: $102.6{ }^{\circ} \mathrm{C} ;{ }^{1} \mathbf{H}$ NMR $\left(300 \mathrm{MHz}, \mathrm{CD}_{2} \mathrm{Cl}_{2}\right): \delta 8.04\left(\mathrm{~m}, 1 \mathrm{H}, \mathrm{H}_{4}\right), 7.77-7.92\left(\mathrm{~m}, 3 \mathrm{H}, \mathrm{H}_{3} \& \mathrm{H}_{9}\right.$ \& $\left.\mathrm{H}_{10}\right), 7.51-7.65\left(\mathrm{~m}, 2 \mathrm{H}, \mathrm{H}_{2} \& \mathrm{H}_{8}\right), 3.18$ (heptd, $\left.{ }^{3} J_{\mathrm{HH}}=7.1 \mathrm{~Hz},{ }^{2} J_{\mathrm{PH}}=11.4 \mathrm{~Hz}, 2 \mathrm{H}, \mathrm{CH} i \mathrm{Pr}\right)$, $1.51\left(\mathrm{~d},{ }^{3} J_{\mathrm{PH}}=8.3 \mathrm{~Hz}, 3 \mathrm{H}, \mathrm{H}_{11}\right), 1.32\left(\mathrm{dd},{ }^{3} J_{\mathrm{HH}}=7.1 \mathrm{~Hz},{ }^{3} J_{\mathrm{PH}}=17.9 \mathrm{~Hz}, 6 \mathrm{H}, \mathrm{CH}_{3} i \mathrm{Pr}\right), 1.20(\mathrm{dd}$, $\left.{ }^{3} J_{\mathrm{HH}}=7.1 \mathrm{~Hz},{ }^{3} J_{\mathrm{PH}}=17.9 \mathrm{~Hz}, 6 \mathrm{H}, \mathrm{CH}_{3} i \mathrm{Pr}\right) ;{ }^{31} \mathbf{P}\left\{{ }^{1} \mathbf{H}\right\} \mathbf{N M R}\left(121 \mathrm{MHz}, \mathrm{CD}_{2} \mathrm{Cl}_{2}\right): \delta 71.9(\mathrm{~s})$; ${ }^{13} \mathbf{C}\left\{{ }^{1} \mathbf{H}\right\}$ NMR (75 MHz, CD $\left.\mathrm{Cl}_{2}\right): \delta 149.0\left(\mathrm{bs}, \mathrm{C}_{7}\right), 135.5\left(\mathrm{~d},{ }^{3} J_{\mathrm{PC}}=14.0 \mathrm{~Hz}, \mathrm{C}_{5}\right), 133.1\left(\mathrm{~d},{ }^{4} J_{\mathrm{PC}}\right.$ $\left.=2.3 \mathrm{~Hz}, \mathrm{C}_{4}\right), 131.4\left(\mathrm{~s}, \mathrm{C}_{3}\right), 130.0\left(\mathrm{~d},{ }^{1} J_{\mathrm{PC}}=58.6 \mathrm{~Hz}, \mathrm{C}_{1}\right), 128.4\left(\mathrm{~s}, \mathrm{C}_{9}\right), 127.7\left(\mathrm{~s}, \mathrm{C}_{10}\right), 127.4$ $\left(\mathrm{s}, \mathrm{C}_{8}\right), 126.3\left(\mathrm{~d},{ }^{2} J_{\mathrm{PC}}=7.3 \mathrm{~Hz}, \mathrm{C}_{2}\right), 27.2\left(\mathrm{~d},{ }^{1} J_{\mathrm{PC}}=24.6 \mathrm{~Hz}, \mathrm{CH} i \mathrm{Pr}\right), 24.8\left(\mathrm{~d},{ }^{2} J_{\mathrm{PC}}=105.6 \mathrm{~Hz}\right.$, $\left.\mathrm{C}_{11}\right), 19.0\left(\mathrm{~d},{ }^{2} \mathrm{~J}_{\mathrm{PC}}=2.8 \mathrm{~Hz}, \mathrm{CH}_{3} i \mathrm{Pr}\right), 18.5\left(\mathrm{~d},{ }^{2} J_{\mathrm{PC}}=1.3 \mathrm{~Hz}, \mathrm{CH}_{3} i \mathrm{Pr}\right.$ ), $\mathrm{C}_{6}$ (not observed); HRMS (ESI+): calcd for $[\mathrm{M}]^{+}=\mathrm{C}_{17} \mathrm{H}_{23} \mathrm{AuP}^{+}$: 455.1203. Found: 455.1213 . 


\section{Reaction of 2a with AgNTf $_{2}$ : Generation of complex 3a and subsequent reactivity}

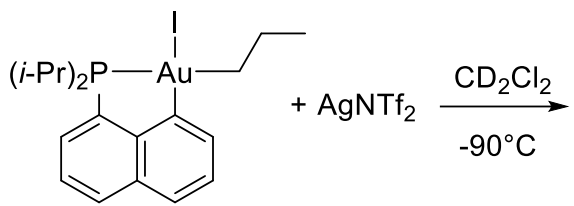

$2 \mathbf{a}$

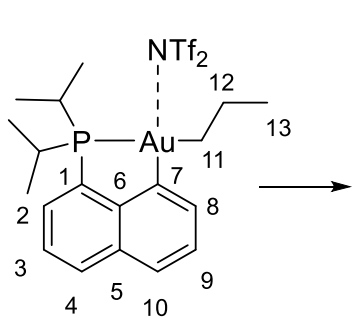

$3 \mathbf{a}$

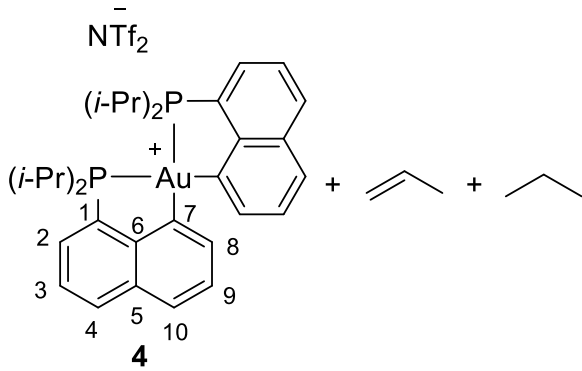

In a glovebox, a screw-cap NMR tube was charged with $\operatorname{AgNTf}_{2}(9.8 \mathrm{mg}, 0.025 \mathrm{mmol})$ in dichloromethane- $\mathrm{d}^{2}(0.35 \mathrm{~mL})$. Complex 2a $(15.4 \mathrm{mg}, 0.025 \mathrm{mmol})$ was transferred into a small glass vial and solubilized in dichloromethane- $\mathrm{d}^{2}(0.35 \mathrm{~mL})$. The prepared solution was loaded into a plastic syringe equipped with a stainless steel needle. The syringe was closed by blocking the needle with a septum. Outside of the glovebox, the NMR tube was put under positive argon pressure and cooled down to $-90{ }^{\circ} \mathrm{C}$ (Acetone/ $\mathrm{N}_{2}$ coldbath). At this temperature, the solution of complex $\mathbf{2 a}$ was added. The tube was kept at $-90{ }^{\circ} \mathrm{C}$ and immediately introduced into the NMR machine for analysis. The reaction was monitored by ${ }^{31} \mathrm{P}$ NMR spectroscopy from $-90^{\circ} \mathrm{C}$ to $0^{\circ} \mathrm{C}$. Complex $3 \mathbf{a}$ was formed at $-90^{\circ} \mathrm{C}$, it was completely characterized at $-50^{\circ} \mathrm{C}$ and it starts to evolve at $-10^{\circ} \mathrm{C}$. Upon warming complex 3a to $0{ }^{\circ} \mathrm{C}$, direct conversion to complex $\mathbf{4}$, propylene and propane was observed. Complex 4 is the only P-containing species detected by ${ }^{31} \mathrm{P}$ NMR. Complex 4 and organic fragments were characterized at $-50^{\circ} \mathrm{C}$. Relative proportions of propylene and propane were determined from integration of the respective resonances and normalization to $[4]=1$.

Complex 3a: ${ }^{1} \mathrm{H}$ NMR (400 MHz, $\left.\mathrm{CD}_{2} \mathrm{Cl}_{2}, 223 \mathrm{~K}\right): \delta 8.13\left(\mathrm{~d},{ }^{3} \mathrm{~J}_{\mathrm{HH}}=7.9 \mathrm{~Hz}, 1 \mathrm{H}, \mathrm{H}_{4}\right), 7.95(\mathrm{dd}$, $\left.{ }^{3} J_{\mathrm{HH}}=8.0 \mathrm{~Hz},{ }^{5} J_{\mathrm{PH}}=1.9 \mathrm{~Hz}, 1 \mathrm{H}, \mathrm{H}_{10}\right), 7.83-7.91\left(\mathrm{~m}, 2 \mathrm{H}, \mathrm{H}_{2} \& \mathrm{H}_{8}\right), 7.68\left(\mathrm{ddd},{ }^{3} J_{\mathrm{HH}}=7.9 \mathrm{~Hz}\right.$, $\left.{ }^{3} J_{\mathrm{HH}}=7.2 \mathrm{~Hz},{ }^{4} J_{\mathrm{PH}}=2.1 \mathrm{~Hz}, 1 \mathrm{H}, \mathrm{H}_{3}\right), 7.62\left(\mathrm{t},{ }^{3} J_{\mathrm{HH}}=8.0 \mathrm{~Hz}, 1 \mathrm{H}, \mathrm{H}_{9}\right), 3.12-3.26(\mathrm{~m}, 2 \mathrm{H}, \mathrm{CH} i \mathrm{Pr})$, 2.33-2.44 (m, 2H, $\left.\mathrm{H}_{11}\right), 1.84-1.98\left(\mathrm{~m}, 2 \mathrm{H}, \mathrm{H}_{12}\right), 1.15-1.37\left(\mathrm{~m}, 12 \mathrm{H}, \mathrm{CH}_{3} i P r\right), 0.97\left(\mathrm{t},{ }^{3} J_{\mathrm{HH}}=7.1\right.$ $\left.\mathrm{Hz}, 3 \mathrm{H}, \mathrm{H}_{13}\right) ;{ }^{31} \mathbf{P}\left\{{ }^{1} \mathbf{H}\right\}$ NMR (162 MHz, $\left.\mathrm{CD}_{2} \mathrm{Cl}_{2}, 223 \mathrm{~K}\right): \delta 74.5(\mathrm{~s}) ;{ }^{19} \mathbf{F}\left\{{ }^{1} \mathbf{H}\right\} \mathbf{N M R}(282 \mathrm{MHz}$, $\left.\mathrm{CD}_{2} \mathrm{Cl}_{2}\right): \delta-79.8(\mathrm{~s}) ;{ }^{13} \mathrm{C}\left\{{ }^{1} \mathbf{H}\right\}$ NMR (100 MHz, $\left.\mathrm{CD}_{2} \mathrm{Cl}_{2}, 223 \mathrm{~K}\right): \delta 147.6\left(\mathrm{bs}, \mathrm{C}_{7}\right), 147.5\left(\mathrm{~d},{ }^{2} J_{\mathrm{PC}}\right.$ $\left.=31.8 \mathrm{~Hz}, \mathrm{C}_{6}\right), 134.8\left(\mathrm{~d},{ }^{3} J_{\mathrm{PC}}=13.6 \mathrm{~Hz}, \mathrm{C}_{5}\right), 133.3\left(\mathrm{~s}, \mathrm{C}_{4}\right), 131.9\left(\mathrm{~s}, \mathrm{C}_{2}\right), 128.9\left(\mathrm{~s}, \mathrm{C}_{10}\right), 128.1$ $\left(\mathrm{s}, \mathrm{C}_{8}\right), 127.5\left(\mathrm{~s}, \mathrm{C}_{9}\right), 126.5\left(\mathrm{~d},{ }^{3} J_{\mathrm{PC}}=7.7 \mathrm{~Hz}, \mathrm{C}_{3}\right), 124.9\left(\mathrm{~d},{ }^{1} J_{\mathrm{PC}}=48.5 \mathrm{~Hz}, \mathrm{C}_{1}\right), 119.0\left(\mathrm{q},{ }^{1} J_{\mathrm{FC}}\right.$ $\left.=321.0 \mathrm{~Hz}, \mathrm{CF}_{3}\right), 55.1\left(\mathrm{~d},{ }^{2} J_{\mathrm{PC}}=88.7 \mathrm{~Hz}, \mathrm{C}_{11}\right), 26.7\left(\mathrm{~d},{ }^{1} J_{\mathrm{PC}}=23.2, \mathrm{CHiPr}\right), 24.1\left(\mathrm{~d},{ }^{3} J_{\mathrm{PC}}=4.4\right.$ $\left.\mathrm{Hz}, \mathrm{C}_{12}\right), 18.6\left(\mathrm{~d},{ }^{2} J_{\mathrm{PC}}=2.0 \mathrm{~Hz}, \mathrm{CH}_{3} i P r\right), 18.5\left(\mathrm{~d},{ }^{4} J_{\mathrm{PC}}=12.0 \mathrm{~Hz}, \mathrm{C}_{13}\right), 18.0\left(\mathrm{~s}, \mathrm{CH}_{3} i P r\right)$. 
Complex 4: ${ }^{1} \mathrm{H}$ NMR $\left(500 \mathrm{MHz}, \mathrm{CD}_{2} \mathrm{Cl}_{2}, 253 \mathrm{~K}\right): \delta 8.17\left(\mathrm{~d},{ }^{3} \mathrm{~J}_{\mathrm{HH}}=7.5 \mathrm{~Hz}, 1 \mathrm{H}, \mathrm{H}_{4}\right), 7.95(\mathrm{~d}$, $\left.{ }^{3} J_{\mathrm{HH}}=7.8 \mathrm{~Hz}, 1 \mathrm{H}, \mathrm{H}_{10}\right), 7.85\left(\mathrm{dd},{ }^{3} J_{\mathrm{HH}}=7.5 \mathrm{~Hz} ;{ }^{3} J_{\mathrm{PH}}=6.9 \mathrm{~Hz} 1 \mathrm{H}, \mathrm{H}_{2}\right), 7.72\left(\mathrm{td},{ }^{3} J_{\mathrm{HH}}=7.5\right.$ $\left.\mathrm{Hz} ;{ }^{4} J_{\mathrm{PH}}=2.5 \mathrm{~Hz}, 1 \mathrm{H}, \mathrm{H}_{3}\right), 7.69\left(\mathrm{dd},{ }^{3} J_{\mathrm{HH}}=7.8 \mathrm{~Hz} ;{ }^{4} J_{\mathrm{PH}}=2.3 \mathrm{~Hz}, 1 \mathrm{H}, \mathrm{H}_{8}\right), 7.62\left(\mathrm{td},{ }^{3} J_{\mathrm{HH}}=7.8\right.$ $\left.\mathrm{Hz} ;{ }^{5} J_{\mathrm{PH}}=2.1 \mathrm{~Hz} 1 \mathrm{H}, \mathrm{H} 9\right), 3.35-3.46(\mathrm{~m}, 2 \mathrm{H}, \mathrm{CHiPr}), 1.28-1.50\left(\mathrm{~m}, 12 \mathrm{H}, \mathrm{CH}_{3} i \mathrm{Pr}\right) ;{ }^{31} \mathbf{P}\left\{{ }^{1} \mathbf{H}\right\}$ NMR (202 MHz, $\left.\mathrm{CD}_{2} \mathrm{Cl}_{2}, 253 \mathrm{~K}\right): \delta 84.9$ (s); ${ }^{13} \mathbf{C}\left\{{ }^{1} \mathbf{H}\right\} \mathbf{N M R}\left(125 \mathrm{MHz}, \mathrm{CD}_{2} \mathrm{Cl}_{2}, 253 \mathrm{~K}\right): \delta 168.5$ $\left(\mathrm{dd},{ }^{2} J_{\mathrm{PC}-\text { trans }}=114.0 \mathrm{~Hz},{ }^{2} J_{\mathrm{PC}}=5.1 \mathrm{~Hz}, \mathrm{C}_{7}\right), 148.3\left(\mathrm{~d},{ }^{2} J_{\mathrm{PC}}=30.0 \mathrm{~Hz}, \mathrm{C}_{6}\right), 136.7\left(\mathrm{~s}, \mathrm{C}_{8}\right), 133.9$ $\left(\mathrm{d},{ }^{3} J_{\mathrm{PC}}=18.6 \mathrm{~Hz}, \mathrm{C}_{5}\right), 133.8\left(\mathrm{~s}, \mathrm{C}_{4}\right), 131.5\left(\mathrm{~s}, \mathrm{C}_{2}\right), 128.5\left(\mathrm{t},{ }^{4} J_{\mathrm{PC}}=3.7 \mathrm{~Hz}, \mathrm{C}_{9}\right), 127.9\left(\mathrm{~s}, \mathrm{C}_{10}\right)$, $126.9\left(\mathrm{dd},{ }^{1} J_{\mathrm{PC}}=54.8 \mathrm{~Hz},{ }^{3} J_{\mathrm{PC}}=6.1 \mathrm{~Hz}, \mathrm{C}_{1}\right), 126.6\left(\mathrm{t}, J_{\mathrm{PC}}=4.7 \mathrm{~Hz}, \mathrm{C}_{3}\right), 119.7\left(\mathrm{q},{ }^{1} J_{\mathrm{FC}}=321.0\right.$ $\mathrm{Hz}, \mathrm{CF}_{3}$ ), 31.6 (bs, $\mathrm{CHiPr}$ ), 18.8 (bs, $\mathrm{CH}_{3} i \mathrm{Pr}$ ), 18.1 (s, $\mathrm{CH}_{3} i \mathrm{Pr}$ ). HRMS (ESI+): calcd for [M] $]^{+}=$ $\mathrm{C}_{32} \mathrm{H}_{40} \mathrm{AuP}_{2}^{+}$: 683.2271 . Found: 683.2285 .

Propylene ( 25\%): ${ }^{1} \mathrm{H}$ NMR (400 MHz, $\left.\mathrm{CD}_{2} \mathrm{Cl}_{2}, 223 \mathrm{~K}\right): 5.84$ (m, 1H, CH), $5.02\left(\mathrm{~m}, 1 \mathrm{H}, \mathrm{CH}_{2}\right.$ ), $4.92\left(\mathrm{~m}, 1 \mathrm{H}, \mathrm{CH}_{2}\right), 1.71\left(\mathrm{ddd},{ }^{3} J_{\mathrm{HH}}=6.5 \mathrm{~Hz},{ }^{4} J_{\mathrm{HH}}=1.5 \mathrm{~Hz},{ }^{4} J_{\mathrm{HH}}=1.5 \mathrm{~Hz}\right)$.

Propane ( 30\%): ${ }^{1} \mathbf{H}$ NMR (400 MHz, $\left.\mathrm{CD}_{2} \mathrm{Cl}_{2}, 223 \mathrm{~K}\right): 1.32\left(\mathrm{~m}, 2 \mathrm{H}, \mathrm{CH}_{2}\right), 0.90\left(\mathrm{t},{ }^{3} \mathrm{~J}_{\mathrm{HH}}=7.3\right.$ $\mathrm{Hz}, 3 \mathrm{H})$. 


\section{Reaction of complex 2b with AgNTf $\mathrm{f}_{2}$ : Generation of complex $3 \mathrm{~b}$ and subsequent reactivity}

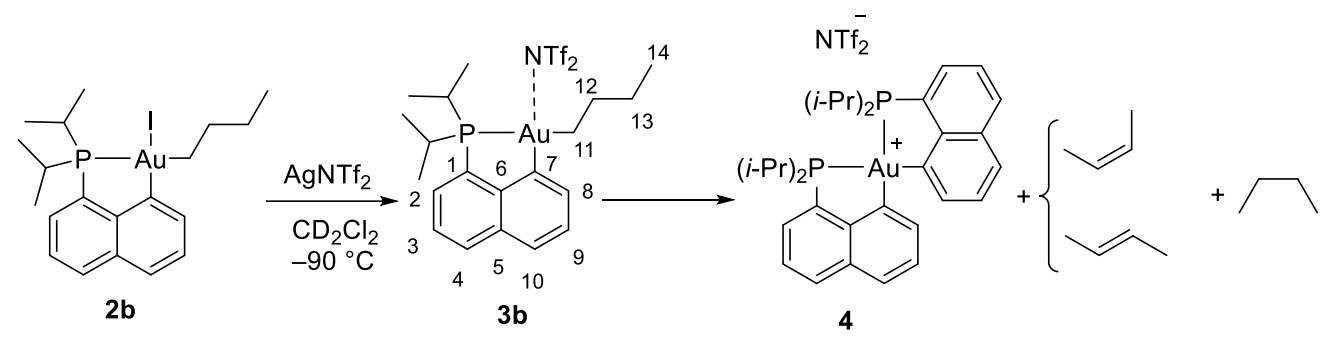

In a glovebox, a screw-cap NMR tube was charged with $\operatorname{AgNTf}_{2}(12.8 \mathrm{mg}, 0.033 \mathrm{mmol})$ in dichloromethane- $\mathrm{d}^{2}(0.35 \mathrm{~mL})$. Complex $2 \mathbf{b}(18.7 \mathrm{mg}, 0.029 \mathrm{mmol})$ was transferred into a small glass vial and solubilized in dichloromethane- $\mathrm{d}^{2}(0.35 \mathrm{~mL})$. The prepared solution was loaded into a plastic syringe equipped with stainless steel needle. The syringe was closed by blocking the needle with a septum. Outside of the glovebox, the NMR tube was put under positive argon pressure and cooled down to $-90{ }^{\circ} \mathrm{C}$ (Acetone/ $\mathrm{N}_{2}$ coldbath). At this temperature, the solution of complex $\mathbf{2 b}$ was added. The tube was kept at $-90{ }^{\circ} \mathrm{C}$ and immediately introduced into the NMR machine for analysis. The reaction was monitored by ${ }^{31} \mathrm{P}$ NMR spectroscopy from $-90^{\circ} \mathrm{C}$ to room temperature. Complex $\mathbf{3 b}$ was formed at $-80^{\circ} \mathrm{C}$, it was completely characterized at $40^{\circ} \mathrm{C}$ and it is stable up to $0^{\circ} \mathrm{C}$. Upon warming complex $3 \mathrm{~b}$ to $10{ }^{\circ} \mathrm{C}$, direct conversion to complex 4, butane and 2-butene was observed. Complex 4 is the only P-containing species detected by ${ }^{31} \mathrm{P}$ NMR. Complex 4 and organic fragments were characterized at $-20^{\circ} \mathrm{C}$. 2 -butene and butane formation was confirmed by NMR and GCMS. Relative proportions of 2-butene and butane were determined from integration of the respective resonances and normalization to [4] $=1$. Cis and trans 2-butene were assigned according to ${ }^{1} \mathrm{H}$ and ${ }^{13} \mathrm{C}$ chemical shifts and ${ }^{3} J_{\mathrm{HH}}$ coupling constant reported in the literature ${ }^{2}$.

Complex 3b: ${ }^{1} \mathrm{H}$ NMR $\left(400 \mathrm{MHz}, \mathrm{CD}_{2} \mathrm{Cl}_{2}, 233 \mathrm{~K}\right): \delta 8.14\left(\mathrm{~d},{ }^{3} J_{\mathrm{HH}}=7.8 \mathrm{~Hz}, 1 \mathrm{H}, \mathrm{H}_{4}\right), 7.95(\mathrm{~d}$, $\left.{ }^{3} J_{\mathrm{HH}}=7.8 \mathrm{~Hz}, 1 \mathrm{H}, \mathrm{H}_{10}\right), 7.84-7.91\left(\mathrm{~m}, 2 \mathrm{H}, \mathrm{H}_{2} \& \mathrm{H}_{8}\right), 7.70\left(\mathrm{~m}, 1 \mathrm{H}, \mathrm{H}_{3}\right), 7.63\left(\mathrm{t},{ }^{3} J_{\mathrm{HH}}=7.8 \mathrm{~Hz}\right.$, $\left.1 \mathrm{H}, \mathrm{H}_{9}\right), 3.12-3.26$ (m, 2H, CHiPr), 2.40 (m, 2H, $\left.\mathrm{H}_{11}\right), 1.84$ (m, 2H, $\left.\mathrm{H}_{12}\right), 1.33$ (m, 2H, $\mathrm{H}_{13}$ ), 1.19-1.36 (m, 12H, CH $\left.\mathrm{CH}_{3} i \mathrm{Pr}\right), 0.83\left(\mathrm{t},{ }^{3} \mathrm{~J}_{\mathrm{HH}}=7.2 \mathrm{~Hz}, 3 \mathrm{H}, \mathrm{H}_{14}\right) ;{ }^{31} \mathbf{P}\left\{{ }^{1} \mathbf{H}\right\} \mathbf{N M R}\left(162 \mathrm{MHz}, \mathrm{CD}_{2} \mathrm{Cl}_{2}\right.$, 233K): $\delta 74.5(\mathrm{~s}) ;{ }^{19} \mathbf{F}\left\{{ }^{1} \mathbf{H}\right\}$ NMR $\left(282 \mathrm{MHz}, \mathrm{CD}_{2} \mathrm{Cl}_{2}, 233 \mathrm{~K}\right): \delta-79.8(\mathrm{~s}) ;{ }^{13} \mathbf{C}\left\{{ }^{1} \mathbf{H}\right\} \mathbf{N M R}(100$ $\left.\mathrm{MHz}, \mathrm{CD}_{2} \mathrm{Cl}_{2}, 233 \mathrm{~K}\right): \delta 147.6\left(\mathrm{~d},{ }^{2} J_{\mathrm{PC}}=31.8 \mathrm{~Hz}, \mathrm{C}_{6}\right), 147.5\left(\mathrm{bs}, \mathrm{C}_{7}\right), 134.9\left(\mathrm{~d},{ }^{3} J_{\mathrm{PC}}=13.6 \mathrm{~Hz}\right.$, $\left.\mathrm{C}_{5}\right), 133.3\left(\mathrm{~s}, \mathrm{C}_{4}\right), 131.9\left(\mathrm{~s}, \mathrm{C}_{2}\right), 128.9\left(\mathrm{~s}, \mathrm{C}_{10}\right), 128.1\left(\mathrm{~s}, \mathrm{C}_{8}\right), 127.6\left(\mathrm{~s}, \mathrm{C}_{9}\right), 126.6\left(\mathrm{~d},{ }^{3} J_{\mathrm{PC}}=7.8\right.$

\footnotetext{
${ }^{2}$ Bigley, D. B.; Journal of the Chemical Society, Perkin Transactions 2: Physical Organic Chemistry, 1976, 6, 701 .
} 
$\left.\mathrm{Hz}, \mathrm{C}_{3}\right), 125.0\left(\mathrm{~d},{ }^{1} J_{\mathrm{PC}}=48.5 \mathrm{~Hz}, \mathrm{C}_{1}\right), 119.7\left(\mathrm{q},{ }^{1} J_{\mathrm{FC}}=321.0 \mathrm{~Hz}, \mathrm{CF}_{3}\right), 53.3\left(\mathrm{~d},{ }^{2} J_{\mathrm{PC}}=87.7 \mathrm{~Hz}\right.$, $\left.\mathrm{C}_{11}\right), 33.1\left(\mathrm{~d},{ }^{3} J_{\mathrm{PC}}=5.0 \mathrm{~Hz}, \mathrm{C}_{12}\right), 27.5\left(\mathrm{~d},{ }^{4} J_{\mathrm{PC}}=23.1 \mathrm{~Hz}, \mathrm{C}_{13}\right), 26.7\left(\mathrm{~d},{ }^{1} J_{\mathrm{PC}}=23.1 \mathrm{~Hz}, \mathrm{CHiPr}\right)$, $18.6\left(\mathrm{~d},{ }^{2} J_{\mathrm{PC}}=2.7 \mathrm{~Hz}, \mathrm{CH}_{3} i \mathrm{Pr}\right), 18.1\left(\mathrm{~s}, \mathrm{CH}_{3} i \mathrm{Pr}\right), 14.2\left(\mathrm{~s}, \mathrm{C}_{14}\right)$.

Butane (50\%): ${ }^{1} \mathbf{H}$ NMR (500 MHz, $\left.\mathrm{CD}_{2} \mathrm{Cl}_{2}, 253 \mathrm{~K}\right): 1.24\left(\mathrm{~m}, 2 \mathrm{H}, \mathrm{CH}_{2}\right), 0.89\left(\mathrm{t},{ }^{3} \mathrm{~J}_{\mathrm{HH}}=7.1 \mathrm{~Hz}\right.$, $\left.3 \mathrm{H}, \mathrm{CH}_{3}\right) .{ }^{13} \mathbf{C}\left\{{ }^{1} \mathbf{H}\right\}$ NMR $\left(125 \mathrm{MHz}, \mathrm{CD}_{2} \mathrm{Cl}_{2}, 253 \mathrm{~K}\right): 25.2\left(\mathrm{~s}, \mathrm{CH}_{2}\right), 14.05\left(\mathrm{~s}, \mathrm{CH}_{3}\right)$.

2-Butene (cis): $(\sim 40 \%){ }^{1} \mathbf{H}$ NMR $\left(500 \mathrm{MHz}, \mathrm{CD}_{2} \mathrm{Cl}_{2}, 253 \mathrm{~K}\right): 5.43(\mathrm{~m}, \mathrm{CH}), 1.57\left(\mathrm{~d},{ }^{3} J_{\mathrm{HH}}=\right.$ $\left.5.2 \mathrm{~Hz}, \mathrm{CH}_{3}\right) .{ }^{13} \mathrm{C}\left\{{ }^{1} \mathbf{H}\right\}$ NMR $\left(125 \mathrm{MHz}, \mathrm{CD}_{2} \mathrm{Cl}_{2}, 253 \mathrm{~K}\right): 124.9(\mathrm{~s}, \mathrm{CH}), 12.6\left(\mathrm{~s}, \mathrm{CH}_{3}\right)$.

2-Butene (trans): $(\sim 10 \%){ }^{1} \mathbf{H}$ NMR (500 MHz, $\left.\mathrm{CD}_{2} \mathrm{Cl}_{2}, 253 \mathrm{~K}\right): 5.41$ (m, CH), 1.59 (m, $\left.\mathrm{CH}_{3}\right)$. ${ }^{13} \mathbf{C}\left\{{ }^{1} \mathbf{H}\right\}$ NMR $\left(125 \mathrm{MHz}, \mathrm{CD}_{2} \mathrm{Cl}_{2}, 253 \mathrm{~K}\right): 125.5$ (s, $\left.\mathrm{CH}\right), 17.9\left(\mathrm{~s}, \mathrm{CH}_{3}\right)$. 
Reaction of complex 2c with AgNTf 2 : Generation of complex 3c

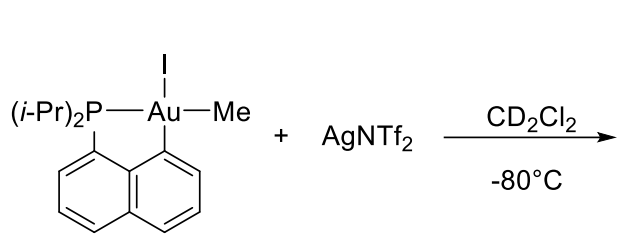

2c

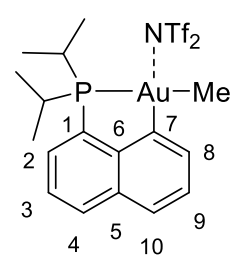

$3 c$

A solution of complex $2 \mathbf{c}(176 \mathrm{mg}, 0.3 \mathrm{mmol})$ in DCM $(6 \mathrm{~mL})$ was added to a solution of $\operatorname{AgNTf}_{2}(117 \mathrm{mg}, 0.3 \mathrm{mmol})$ in DCM $(6 \mathrm{~mL})$ at $-80^{\circ} \mathrm{C}$. The reaction was allowed to warm up to room temperature and filtered over celite. The solvent was removed in vacuum and the residue was washed with pentane to afford 3c as a white solid. Yield: $100 \mathrm{mg}$ (77\%).

${ }^{1} \mathbf{H}$ NMR $\left(300 \mathrm{MHz}, \mathrm{CD}_{2} \mathrm{Cl}_{2}, 298 \mathrm{~K}\right): \delta 8.06\left(\mathrm{~d},{ }^{3} \mathrm{~J}_{\mathrm{HH}}=8.0 \mathrm{~Hz}, 1 \mathrm{H}, \mathrm{H}_{4}\right), 7.80-7.87\left(\mathrm{~m}, 2 \mathrm{H}, \mathrm{H}_{8}\right.$ \& $\left.\mathrm{H}_{10}\right), 7.73\left(\mathrm{~m}, 1 \mathrm{H}, \mathrm{H}_{2}\right), 7.65\left(\mathrm{dt},{ }^{3} J_{\mathrm{HH}}=8.0 \mathrm{~Hz},{ }^{4} J_{\mathrm{PH}}=1.8 \mathrm{~Hz}, 1 \mathrm{H}, \mathrm{H}_{3}\right), 7.50\left(\mathrm{~m}, 1 \mathrm{H}, \mathrm{H}_{9}\right)$, $3.20\left(\right.$ heptd, $\left.{ }^{3} J_{\mathrm{HH}}=7.0 \mathrm{~Hz},{ }^{2} J_{\mathrm{PH}}=11.1 \mathrm{~Hz}, 2 \mathrm{H}, \mathrm{CH} i \mathrm{Pr}\right), 1.53\left(\mathrm{~d},{ }^{3} J_{\mathrm{PH}}=8.1 \mathrm{~Hz}, 3 \mathrm{H}, \mathrm{Me}\right), 1.22$ $\left(\mathrm{dd},{ }^{3} J_{\mathrm{HH}}=7.0 \mathrm{~Hz},{ }^{3} J_{\mathrm{PH}}=16.9 \mathrm{~Hz}, 6 \mathrm{H}, \mathrm{CH}_{3} i \mathrm{Pr}\right), 1.20\left(\mathrm{dd},{ }^{3} J_{\mathrm{HH}}=7.0 \mathrm{~Hz},{ }^{3} J_{\mathrm{PH}}=16.9 \mathrm{~Hz}, 6 \mathrm{H}\right.$, $\left.\mathrm{CH}_{3} i \mathrm{Pr}\right) ;{ }^{31} \mathbf{P}\left\{{ }^{1} \mathbf{H}\right\}$ NMR $\left(121 \mathrm{MHz}, \mathrm{CD}_{2} \mathrm{Cl}_{2}, 298 \mathrm{~K}\right): \delta 78.6(\mathrm{~s}) ;{ }^{19} \mathbf{F}\left\{{ }^{1} \mathbf{H}\right\} \mathbf{N M R}(282 \mathrm{MHz}$, $\left.\mathrm{CD}_{2} \mathrm{Cl}_{2}\right): \delta-74.2(\mathrm{bs}) ;{ }^{13} \mathrm{C}\left\{{ }^{1} \mathbf{H}\right\}$ NMR $\left(75 \mathrm{MHz}, \mathrm{CD}_{2} \mathrm{Cl}_{2}, 298 \mathrm{~K}\right): \delta 147.9\left(\mathrm{~d},{ }^{2} \mathrm{~J}_{\mathrm{PC}}=28.8 \mathrm{~Hz}, \mathrm{C}_{6}\right)$, $135.3\left(\mathrm{~d},{ }^{3} J_{\mathrm{PC}}=12.9 \mathrm{~Hz}, \mathrm{C}_{5}\right), 133.4\left(\mathrm{~s}, \mathrm{C}_{4}\right), 131.4\left(\mathrm{~s}, \mathrm{C}_{2}\right), 130.7\left(\mathrm{~s}, \mathrm{C}_{10}\right), 129.1\left(\mathrm{~s}, \mathrm{C}_{8}\right), 127.8$ (s, $\left.\mathrm{C}_{9}\right), 126.4\left(\mathrm{~d},{ }^{3} J_{\mathrm{PC}}=7.5 \mathrm{~Hz}, \mathrm{C}_{3}\right), 123.1\left(\mathrm{bs}, \mathrm{C}_{1}\right), 119.9\left(\mathrm{q},{ }^{1} J_{\mathrm{FC}}=321.5 \mathrm{~Hz}, \mathrm{CF}_{3}\right), 34.6\left(\mathrm{~d},{ }^{2} J_{\mathrm{PC}}\right.$ $=92.7 \mathrm{~Hz}, \mathrm{Me}), 25.6\left(\mathrm{~d},{ }^{1} J_{\mathrm{PC}}=21.2 \mathrm{~Hz}, \mathrm{CHiPr}\right), 18.2\left(\mathrm{~d},{ }^{2} J_{\mathrm{PC}}=3.7 \mathrm{~Hz}, \mathrm{CH}_{3} i P r\right), 17.4(\mathrm{~s}$, $\mathrm{CH}_{3} \mathrm{iPr}$ ), $\mathrm{C}_{7}$ (not observed). 


\section{Reaction of complex $3 \mathrm{c}$ with ethylene}

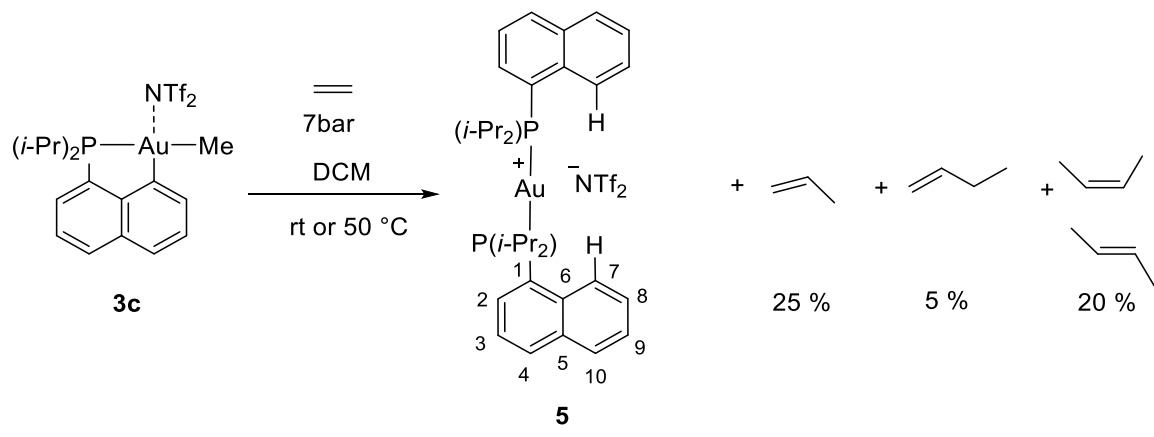

In a pressure tube, complex 3c (16 mg, $0.022 \mathrm{mmol})$ was dissolved in DCM (0.6 mL). The solution was degassed 3 times using the Freeze-Pump-Thaw degassing technique and 7 bar of ethylene were added. The reaction was monitored by NMR. After $1 \mathrm{~h}$ at $50^{\circ} \mathrm{C}$, complex 5 and organic fragment were directly formed. Complex $\mathbf{5}$ is the only P-containing species detected by ${ }^{31} \mathrm{P}$ NMR. The products were characterized at room temperature. Relative proportions of butenes and propylene were determined from integration of the respective resonances and normalization to $[\mathbf{5}]=1$.

Complex 5: ${ }^{1} \mathrm{H}$ NMR $\left(500 \mathrm{MHz}, \mathrm{CD}_{2} \mathrm{Cl}_{2}, 298 \mathrm{~K}\right): \delta 8.92\left(\mathrm{bs}, 1 \mathrm{H}, \mathrm{H}_{7}\right), 8.13\left(\mathrm{~d}, 1 \mathrm{H},{ }^{3} \mathrm{~J}_{\mathrm{HH}}=8.0\right.$ $\left.\mathrm{Hz}, 1 \mathrm{H}, \mathrm{H}_{4}\right), 8.00\left(\mathrm{~m}, 1 \mathrm{H}, \mathrm{H}_{10}\right), 7.95\left(\mathrm{~m}, 1 \mathrm{H}, \mathrm{H}_{2}\right), 7.58-7.71\left(\mathrm{~m}, 3 \mathrm{H}_{1} \mathrm{H}_{3}\right.$ \& $\mathrm{H}_{8}$ \& $\left.\mathrm{H}_{9}\right), 2.78-2.88$ $(\mathrm{m}, 2 \mathrm{H}, \mathrm{CH} i \mathrm{Pr}), 1.40\left(\mathrm{dd},{ }^{3} J_{\mathrm{PH}}=19.6 \mathrm{~Hz} ;{ }^{3} J_{\mathrm{HH}}=6.9 \mathrm{~Hz}, 6 \mathrm{H}, \mathrm{CH}_{3} i P r\right), 1.08\left(\mathrm{dd},{ }^{3} J_{\mathrm{PH}}=18.6\right.$ $\left.\mathrm{Hz} ;{ }^{3} J_{\mathrm{HH}}=6.9 \mathrm{~Hz}, 6 \mathrm{H}, \mathrm{CH}_{3} i \mathrm{Pr}\right) .{ }^{\mathbf{3 1}} \mathbf{P}\left\{{ }^{1} \mathbf{H}\right\} \mathbf{N M R}\left(202 \mathrm{MHz}, \mathrm{CD}_{2} \mathrm{Cl}_{2}, 298 \mathrm{~K}\right): \delta 60(\mathrm{bs}) ;{ }^{13} \mathbf{C}\left\{{ }^{1} \mathbf{H}\right\}$ NMR (125 MHz, $\left.\mathrm{CD}_{2} \mathrm{Cl}_{2}\right): \delta 136.2\left(\mathrm{~s}, \mathrm{C}_{2}\right), 134.9\left(\mathrm{~d},{ }^{3} J_{\mathrm{PC}}=7.3 \mathrm{~Hz}, \mathrm{C}_{5}\right), 134.5\left(\mathrm{~d},{ }^{2} J_{\mathrm{PC}}=7.6 \mathrm{~Hz}\right.$, $\left.\mathrm{C}_{6}\right), 134.4\left(\mathrm{~d},{ }^{4} J_{\mathrm{PC}}=2.7 \mathrm{~Hz}, \mathrm{C}_{4}\right), 134.0$ (bs, $\left.\mathrm{C}_{1}\right), 130.3$ (s, $\left.\mathrm{C}_{10}\right), 128.4$ (s, $\left.\mathrm{C}_{8}\right), 127.5$ (s, $\left.\mathrm{C}_{9}\right), 125.9$ $\left(\mathrm{d},{ }^{3} J_{\mathrm{PC}}=10.2 \mathrm{~Hz}, \mathrm{C}_{7}\right), 125.3\left(\mathrm{~d},{ }^{3} J_{\mathrm{PC}}=12.2 \mathrm{~Hz}, \mathrm{C}_{3}\right), 120.0\left(\mathrm{q},{ }^{1} J_{\mathrm{FC}}=322.6 \mathrm{~Hz}\right), 26.9\left(\mathrm{~d},{ }^{1} J_{\mathrm{PC}}=\right.$ $35.5 \mathrm{~Hz}, \mathrm{CH} i \mathrm{Pr}$ ), 21.2 (s, $\mathrm{CH}_{3} i \mathrm{Pr}$ ), 19.9 (s, $\mathrm{CH}_{3} i \mathrm{Pr}$ ). HRMS (ESI+): calcd for [M] $]^{+}=$ $\mathrm{C}_{32} \mathrm{H}_{42} \mathrm{AuP}_{2}^{+}$: 685.2427 . Found: 685.2429

Propylene (NMR spectroscopic ratio relative to naphtyl signals $25 \%):{ }^{1} \mathbf{H}$ NMR $(500 \mathrm{MHz}$, $\mathrm{CD}_{2} \mathrm{Cl}_{2}$ ): 5.80-5.90 (m, 1H, CH), 5.00-5.06 (m, 1H, $\left.\mathrm{CH}_{2}\right), 4.91-4.95\left(\mathrm{~m}, 1 \mathrm{H}, \mathrm{CH}_{2}\right), 1.72$ (ddd, $\left.{ }^{3} J_{\mathrm{HH}}=6.5 \mathrm{~Hz},{ }^{4} J_{\mathrm{HH}}=1.6 \mathrm{~Hz},{ }^{4} J_{\mathrm{HH}}=1.4 \mathrm{~Hz}\right) ;{ }^{13} \mathbf{C}\left\{{ }^{1} \mathbf{H}\right\} \mathbf{N M R}\left(125 \mathrm{MHz}, \mathrm{CD}_{2} \mathrm{Cl}_{2}\right): 134.2(\mathrm{~s}, \mathrm{CH})$, $115.8\left(\mathrm{~s}, \mathrm{CH}_{2}\right), 19.6\left(\mathrm{~s}, \mathrm{CH}_{3}\right)$.

1-butene (5\%): ${ }^{1} \mathbf{H}$ NMR: $5.60(\mathrm{~m}, \mathrm{CH}), 5.04\left(\mathrm{~m}, \mathrm{CH}_{\text {2olefin }}\right), 2.07\left(\mathrm{~m}, \mathrm{CH}_{2}\right), 1.00$ (t, $J_{\mathrm{HH}}=7.5$ $\left.\mathrm{Hz}, \mathrm{CH}_{3}\right) ;{ }^{13} \mathbf{C}\left\{{ }^{1} \mathbf{H}\right\}$ NMR: 141.1 (s, CH), 122.3 (s, $\left.\mathrm{CH}_{2 \text { olefin }}\right), 27.2\left(\mathrm{~s}, \mathrm{CH}_{2}\right), 13.3\left(\mathrm{~s}, \mathrm{CH}_{3}\right)$. 
2-butene (mixture of cis and trans 20\%): ${ }^{1} \mathbf{H}$ NMR: $5.48(\mathrm{~m}, \mathrm{CH}), 5.44(\mathrm{~m}, \mathrm{CH}), 1.62-1.64(\mathrm{~m}$, $\left.\mathrm{CH}_{3}\right), 1.60-1.62\left(\mathrm{~m}, \mathrm{CH}_{3}\right) ;{ }^{13} \mathbf{C}\left\{{ }^{1} \mathbf{H}\right\}$ NMR: $126.3(\mathrm{~s}, \mathrm{CH}), 125.1(\mathrm{~s}, \mathrm{CH}), 18.2\left(\mathrm{~s}, \mathrm{CH}_{3}\right), 12.7$ (s, $\mathrm{CH}_{3}$ ). 


\section{NMR spectra of complexes}

Figure S1. ${ }^{1} \mathrm{H}$ NMR spectrum of complex $2 \mathbf{a}$

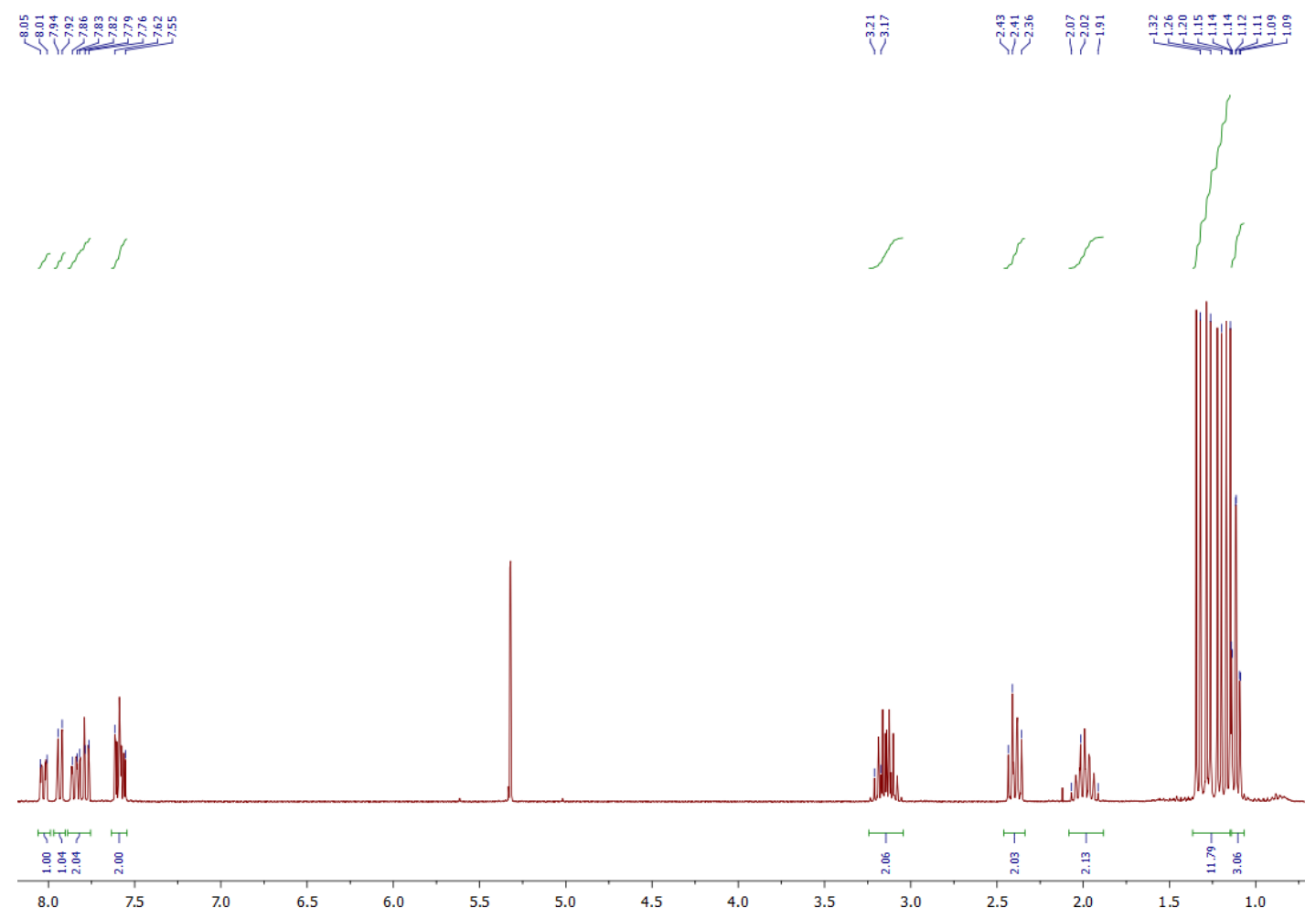

Figure S2 ${ }^{31} \mathrm{P}\left\{{ }^{1} \mathrm{H}\right\}$ NMR spectrum of complex 2a

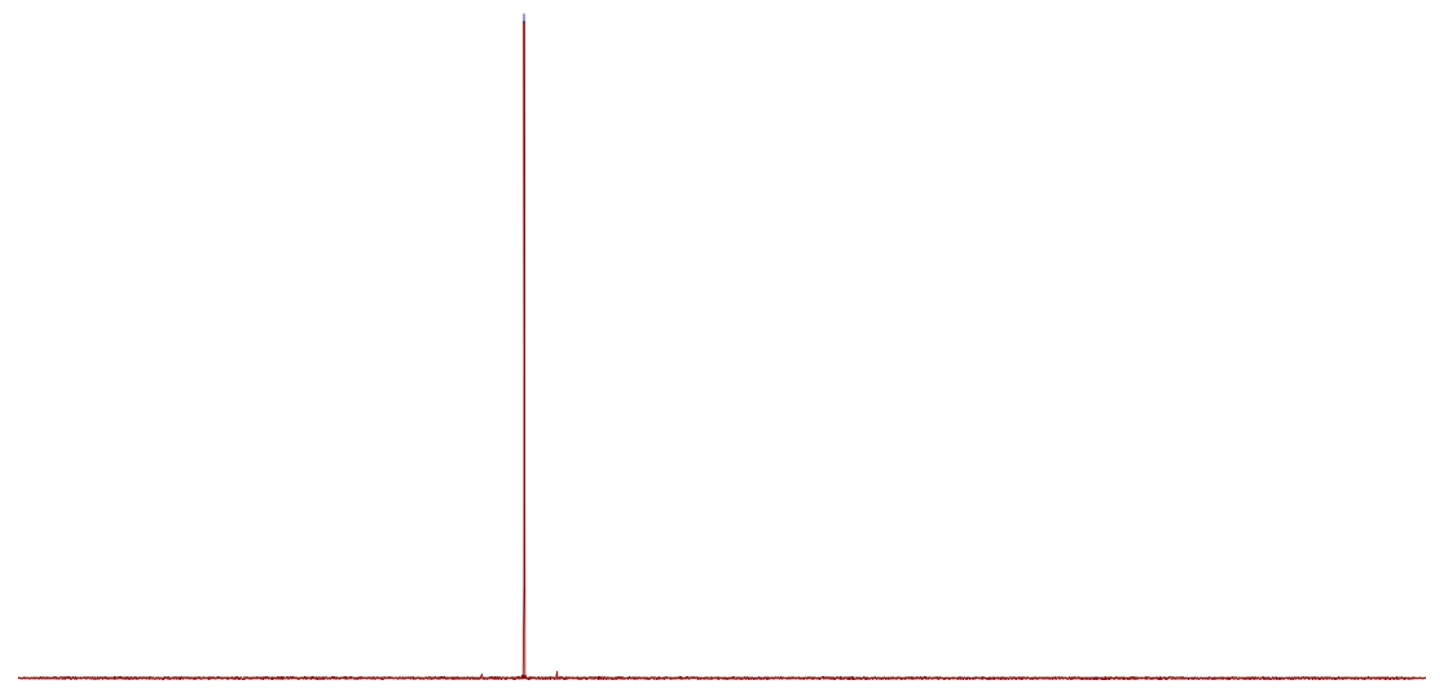

$\begin{array}{llllllllllllllllllllllll}2 & 180 & 180 & 160 & 140 & 120 & 100 & 80 & 60 & 40 & 20 & 0 & -20 & -40 & -60 & -80 & -100 & -120 & -140 & -160 & -180\end{array}$ 
Figure S3. ${ }^{13} \mathrm{C}\left\{{ }^{1} \mathrm{H}\right\}$ NMR spectrum of complex 2a
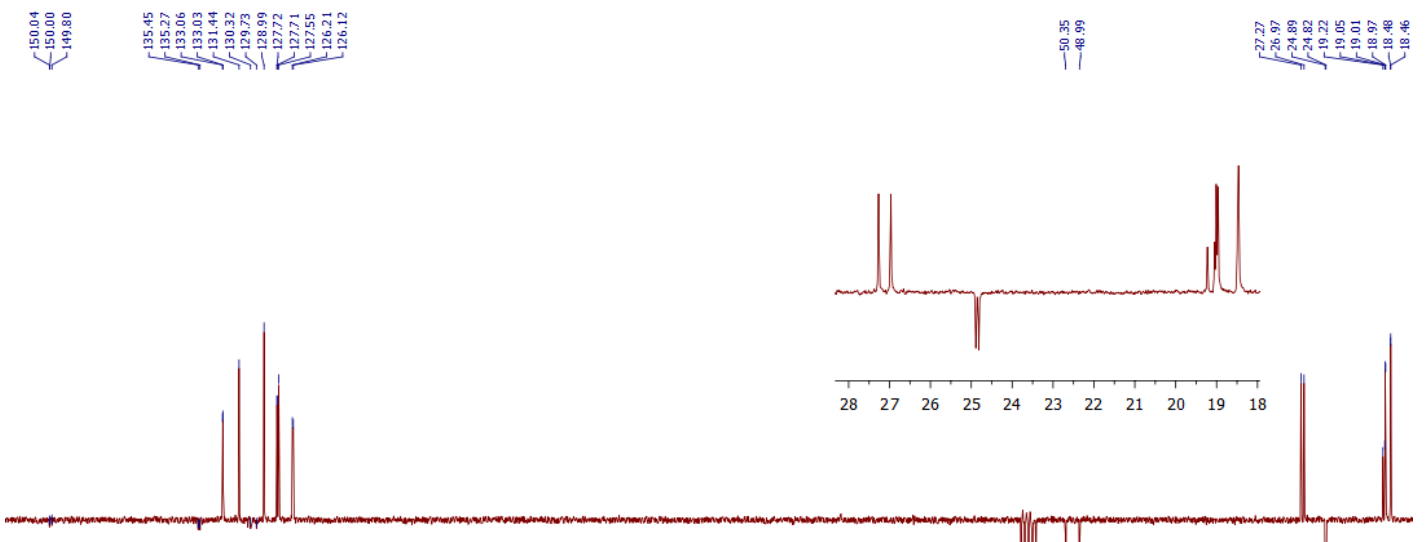

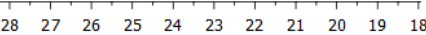

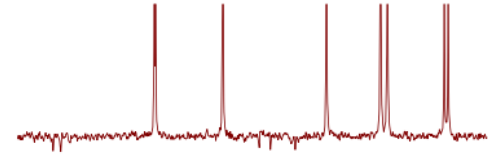

$\begin{array}{lllllllllll}136 & 135 & 134 & 133 & 132 & 131 & 130 & 129 & 128 & 127 & 126\end{array}$

$\begin{array}{llllllllllllllllllllllllllllll}150 & 145 & 140 & 135 & 130 & 125 & 120 & 115 & 110 & 105 & 100 & 95 & 90 & 85 & 80 & 75 & 70 & 65 & 60 & 55 & 50 & 45 & 40 & 35 & 30 & 25 & 20\end{array}$

Figure S4. ${ }^{1} \mathrm{H}$ NMR spectrum of complex $\mathbf{2 b}$

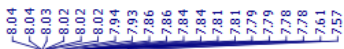

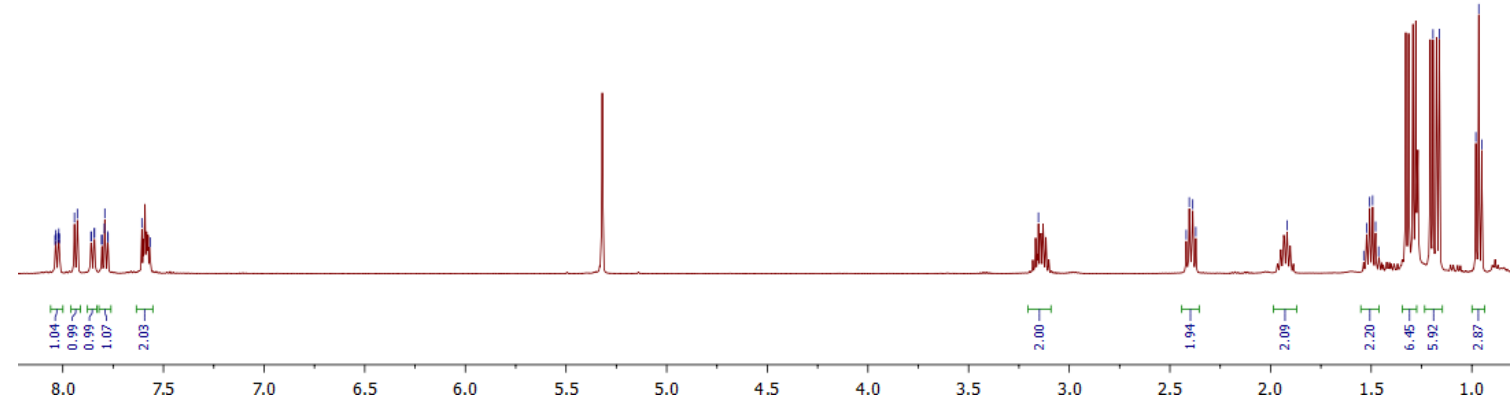


Figure S5. ${ }^{31} \mathrm{P}\left\{{ }^{1} \mathrm{H}\right\}$ NMR spectrum of complex $\mathbf{2 b}$

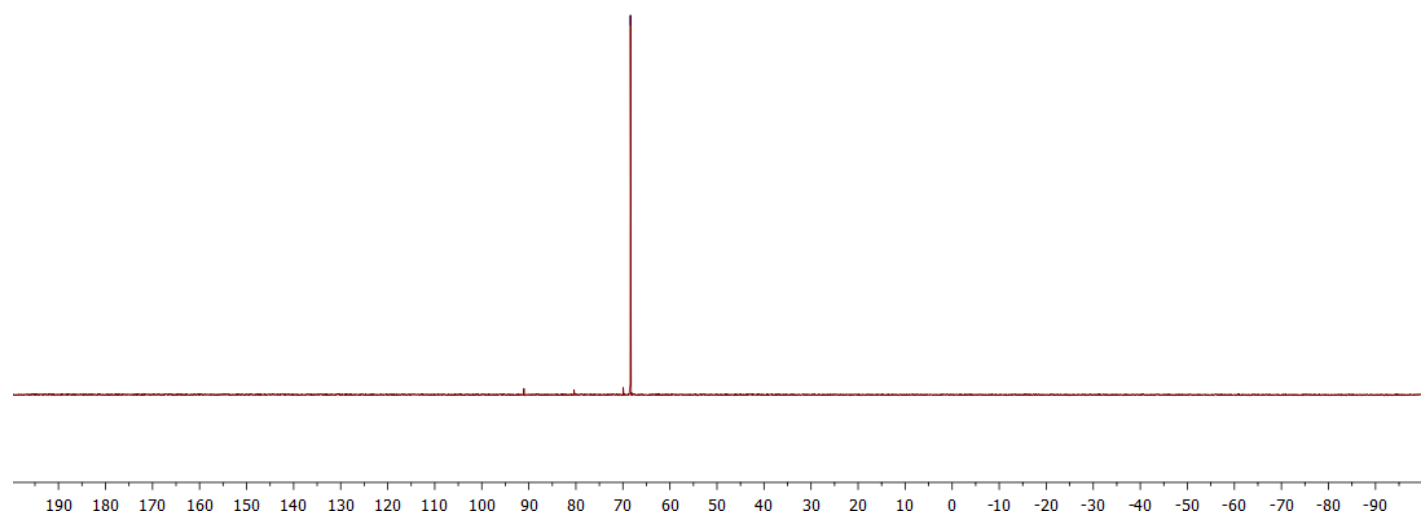

Figure S6. ${ }^{13} \mathrm{C}\left\{{ }^{1} \mathrm{H}\right\}$ NMR spectrum of complex $\mathbf{2 b}$

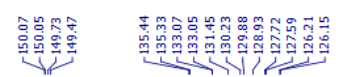

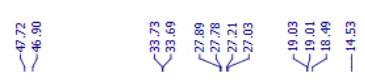

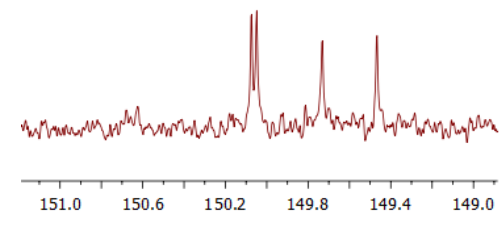

$\begin{array}{llllllllllllllllllllllllllllllllllllllllll}150 & 145 & 140 & 135 & 130 & 125 & 120 & 115 & 110 & 10 & 100 & 95 & 90 & 85 & 80 & 75 & 70 & 65 & 60 & 55 & 50 & 45 & 40 & 35 & 30 & 25 & 20 & 15 & 16\end{array}$ 
Figure S7. ${ }^{1} \mathrm{H}$ NMR spectrum of complex $2 \mathrm{c}$

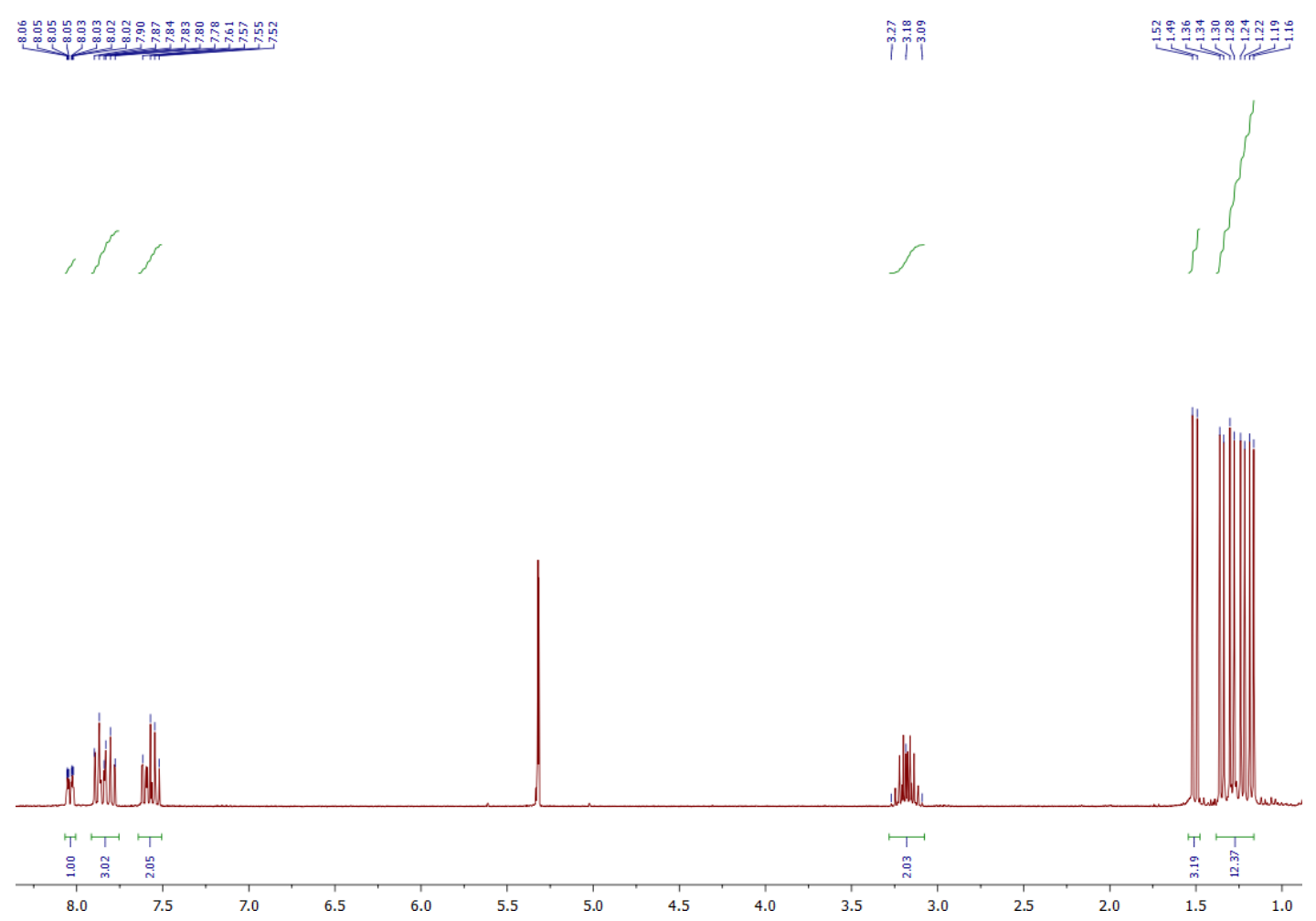

Figure S8. ${ }^{31} \mathrm{P}\left\{{ }^{1} \mathrm{H}\right\}$ NMR spectrum of complex $2 \mathrm{c}$

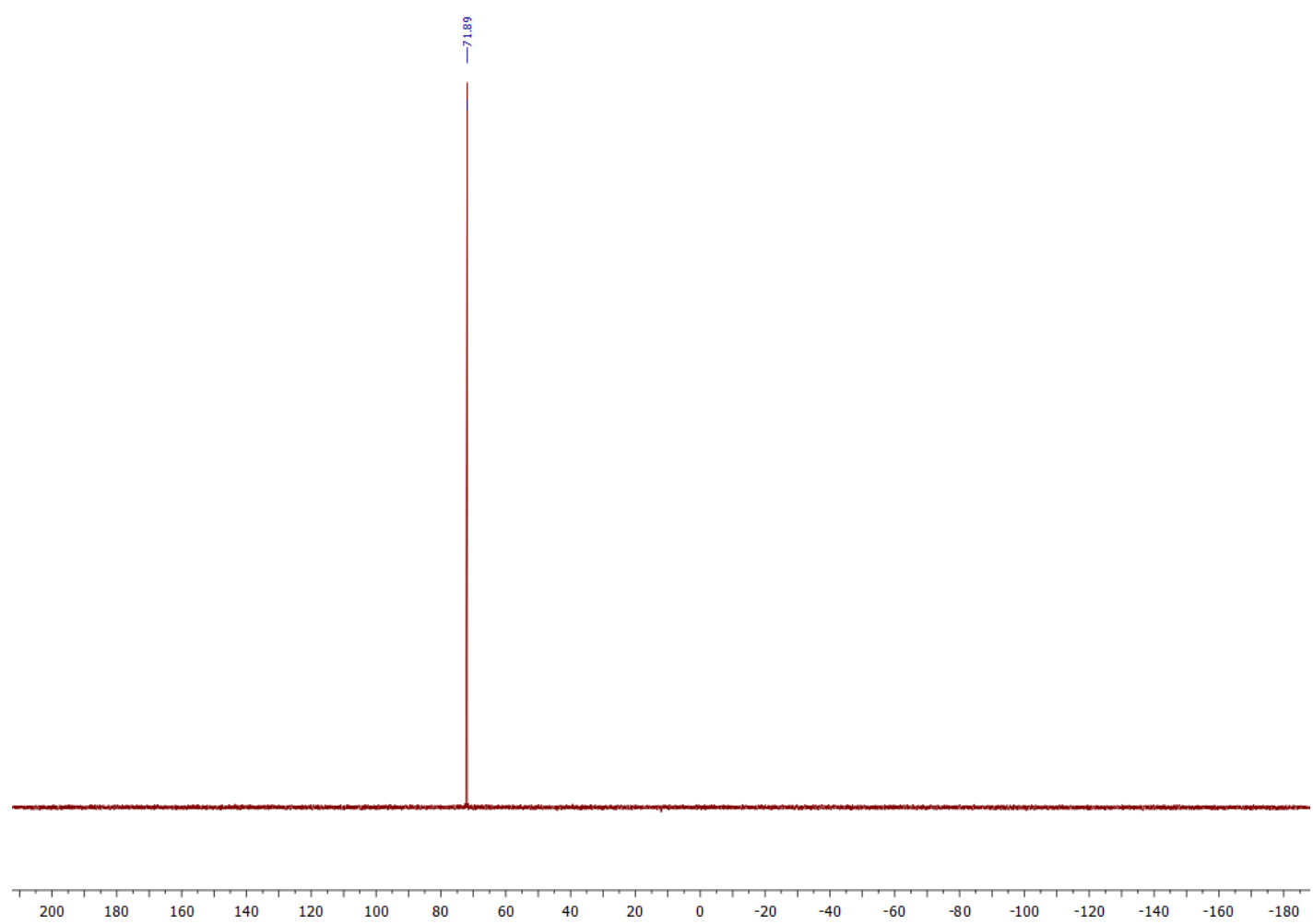


Figure S9. ${ }^{13} \mathrm{C}\left\{{ }^{1} \mathrm{H}\right\}$ NMR spectrum of complex 2c

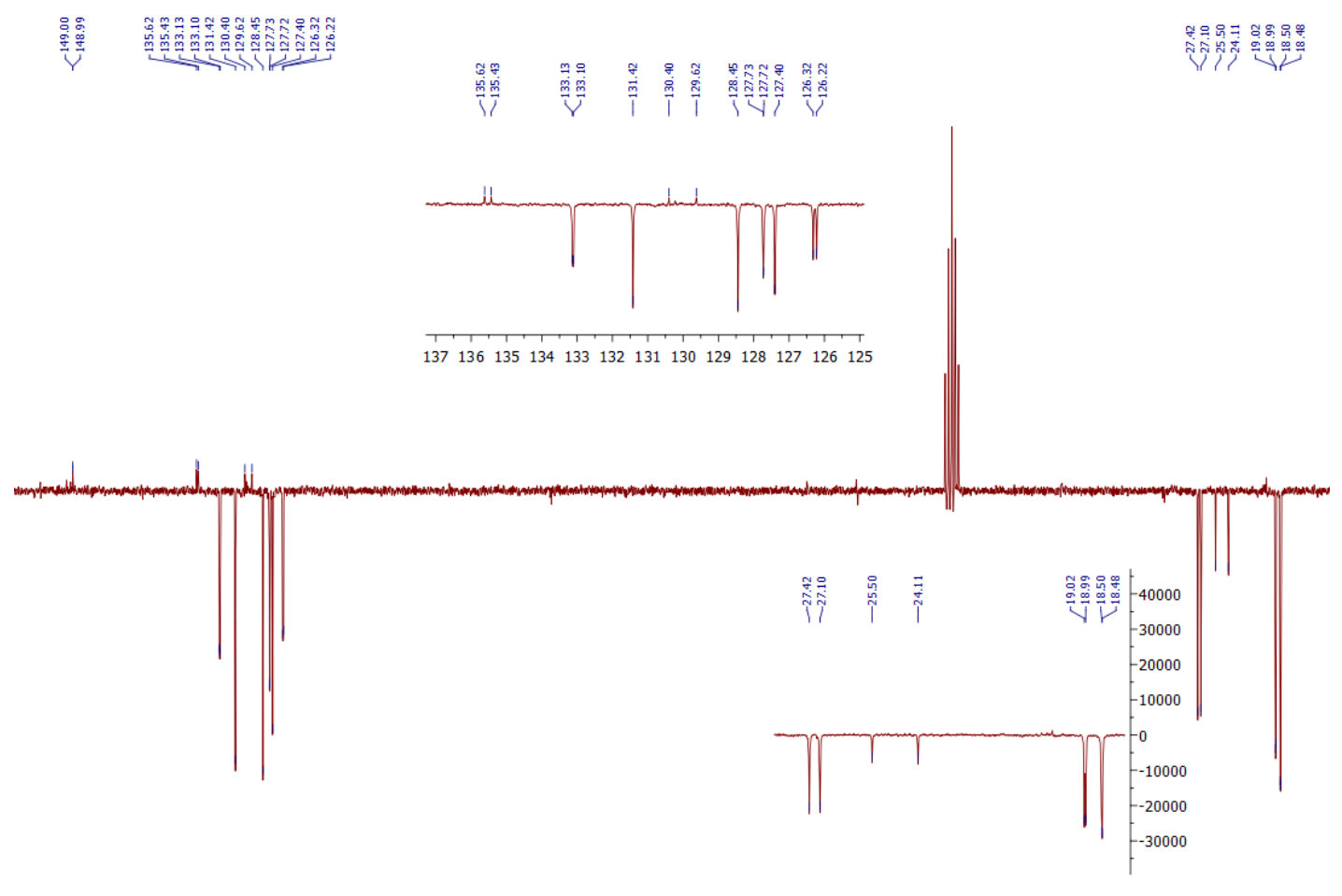

$\begin{array}{lllllllllllllllllllllllllllllllllllllllllll}55 & 150 & 145 & 140 & 135 & 130 & 125 & 120 & 115 & 110 & 105 & 100 & 95 & 90 & 85 & 80 & 75 & 70 & 65 & 60 & 55 & 50 & 45 & 40 & 35 & 30 & 25 & 20 & 15\end{array}$

Figure S10. ${ }^{1} \mathrm{H}$ NMR spectrum of complex 3a

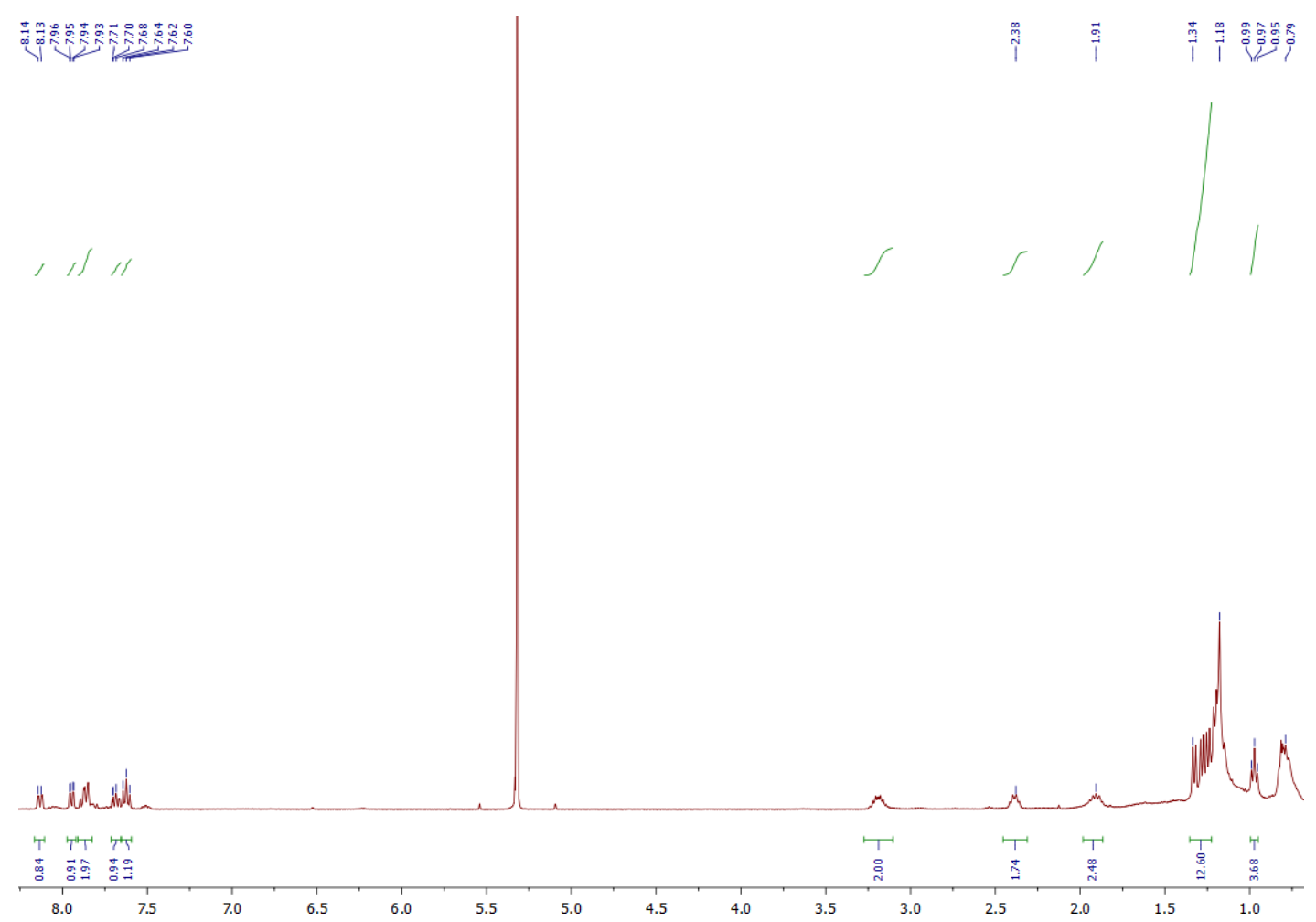


Figure S11. ${ }^{31} \mathrm{P}\left\{{ }^{1} \mathrm{H}\right\}$ NMR spectrum of complex 3a

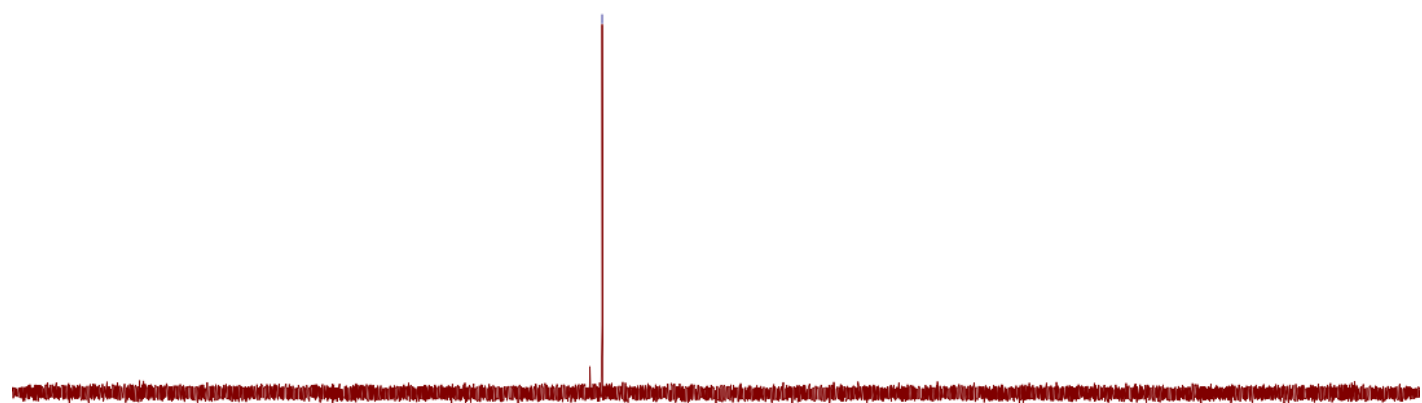

$\begin{array}{llllllllllllllllllllllllllllllllll}0 & 00 & 190 & 180 & 170 & 160 & 150 & 140 & 130 & 120 & 110 & 100 & 90 & 80 & 70 & 60 & 50 & 40 & 30 & 20 & 10 & 0 & -10 & -20 & -30 & -40 & -50 & -60 & -70 & -80 & -90 & -10\end{array}$

Figure S12. ${ }^{13} \mathrm{C}\left\{{ }^{1} \mathrm{H}\right\}$ NMR spectrum of complex 3a

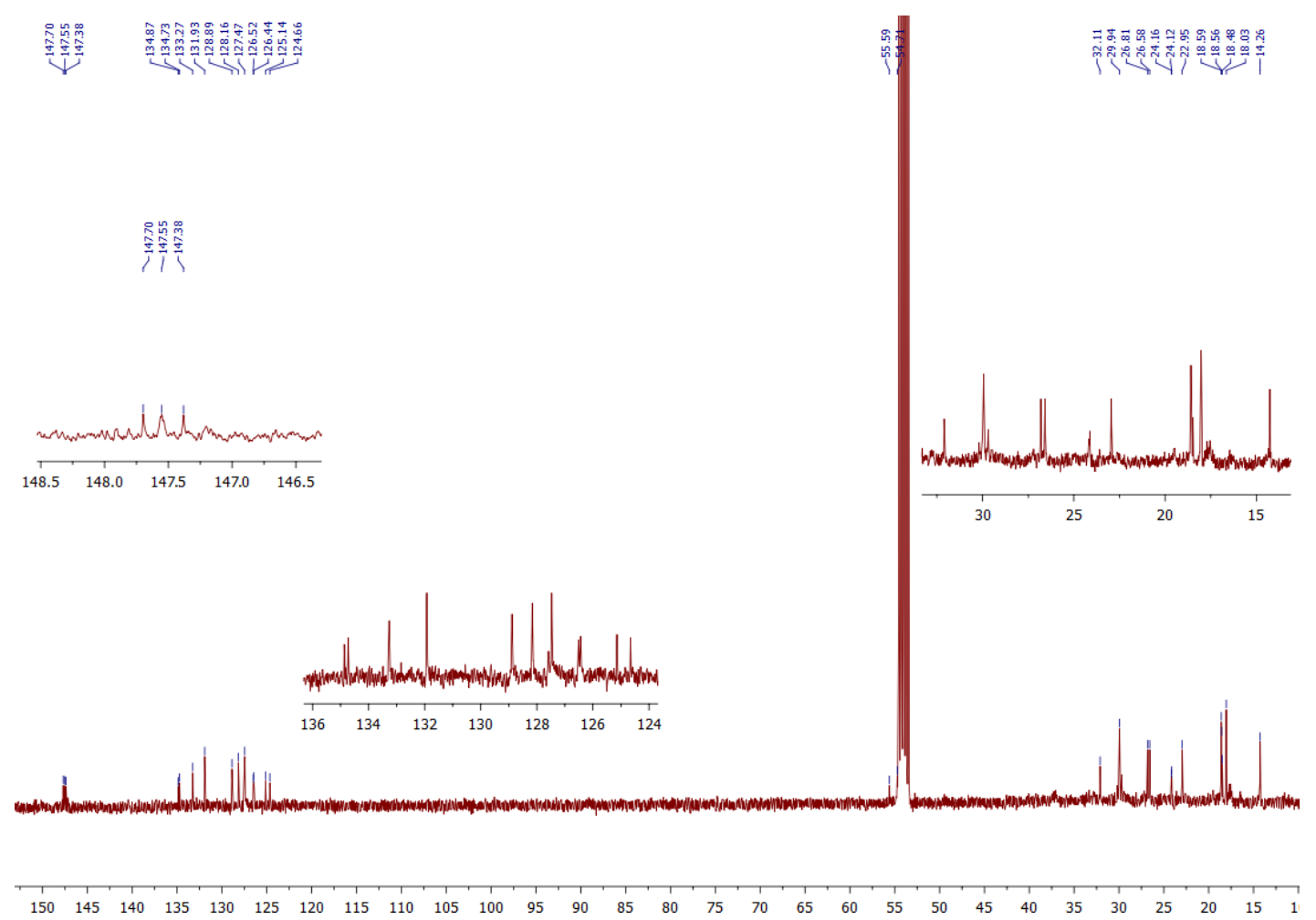


Figure S13. ${ }^{1} \mathrm{H}$ NMR spectrum of complex 4 with propane (*) and propene (arrows).

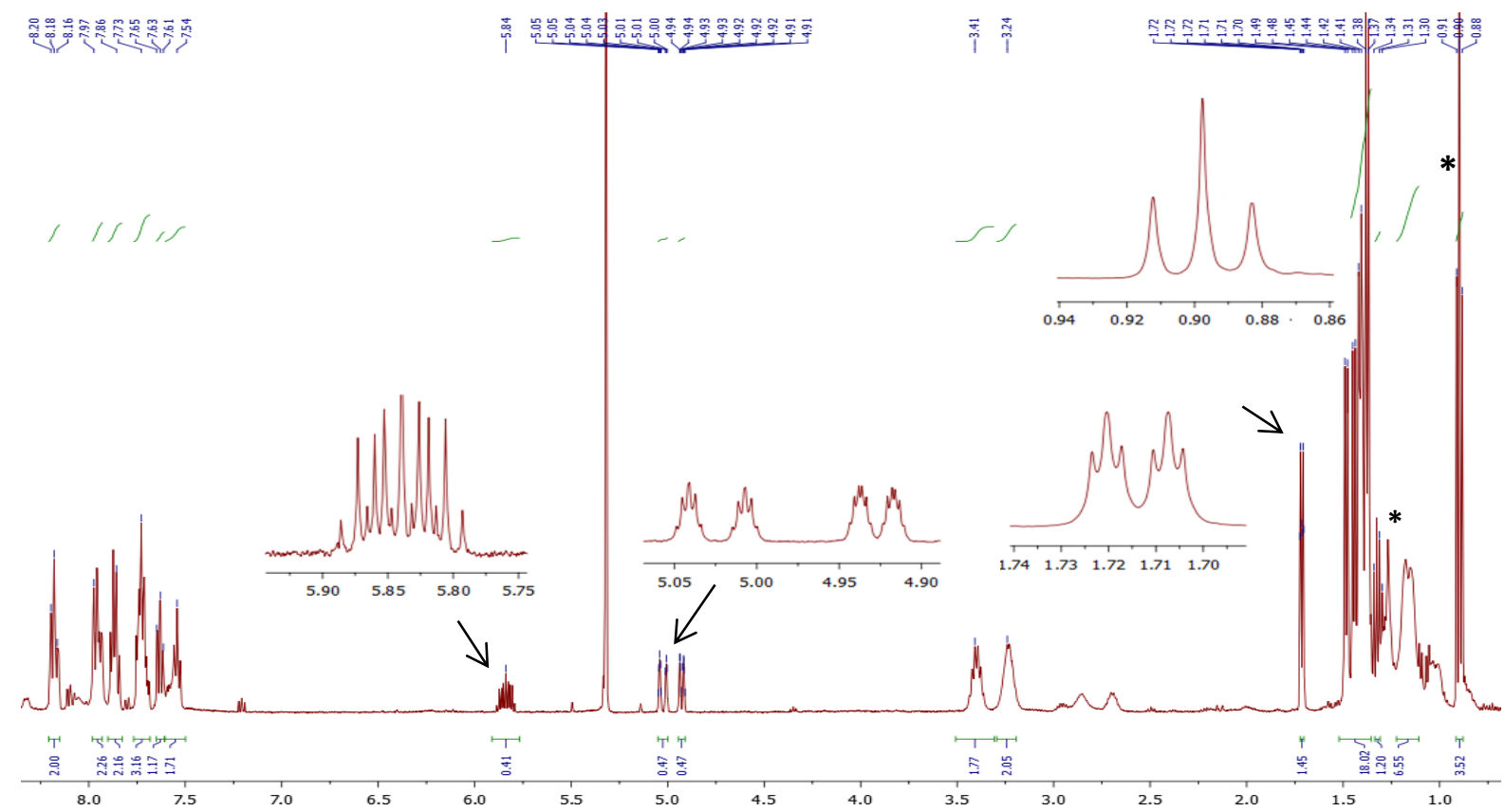

Figure S14. ${ }^{31} \mathrm{P}\left\{{ }^{1} \mathrm{H}\right\}$ NMR spectrum of complex 4 
Figure S15. ${ }^{13} \mathrm{C}\left\{{ }^{1} \mathrm{H}\right\}$ NMR spectrum of complex 4 with propane and propene
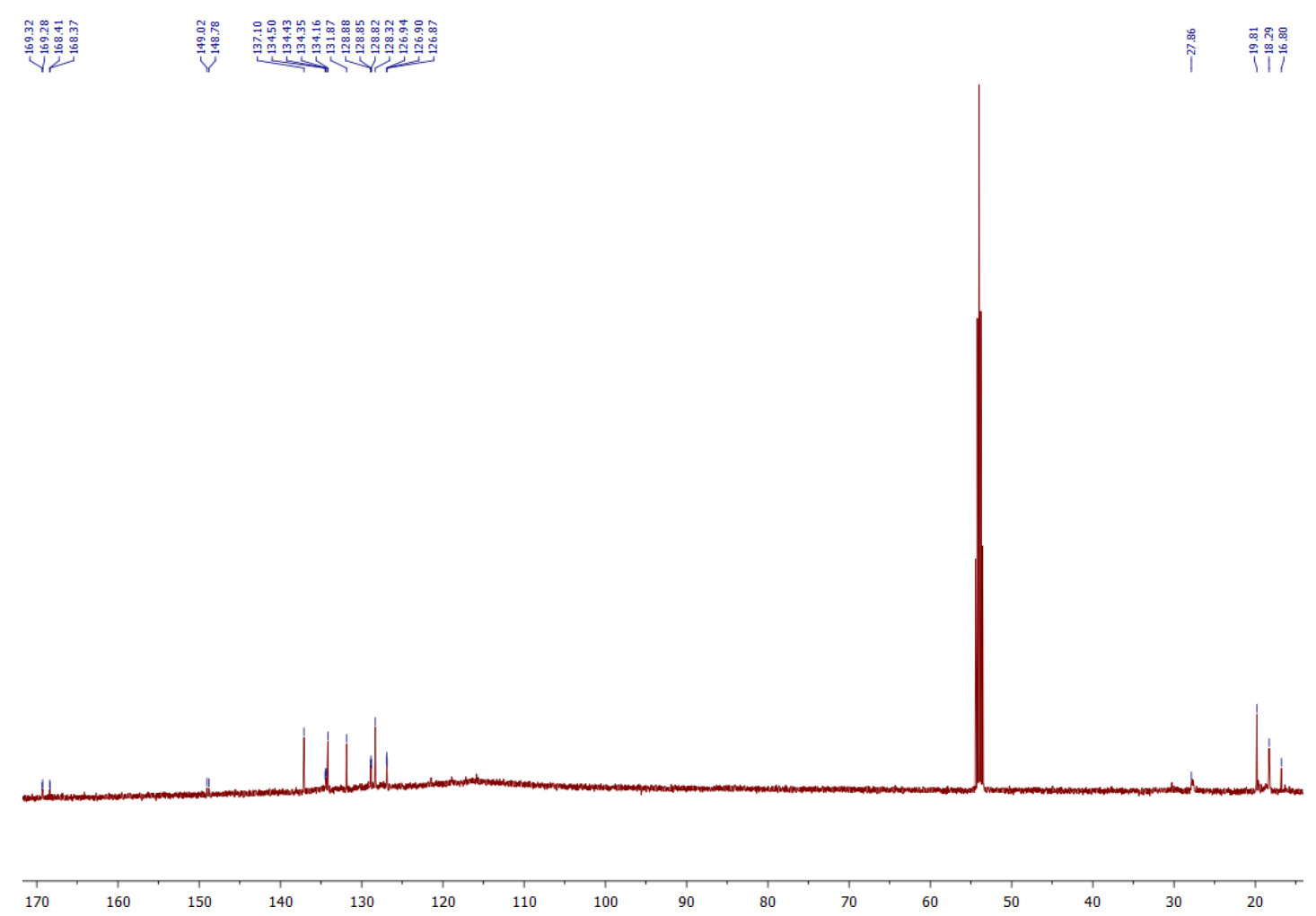

Figure S16. ${ }^{1} \mathrm{H}$ NMR spectrum of complex $\mathbf{3 b}$

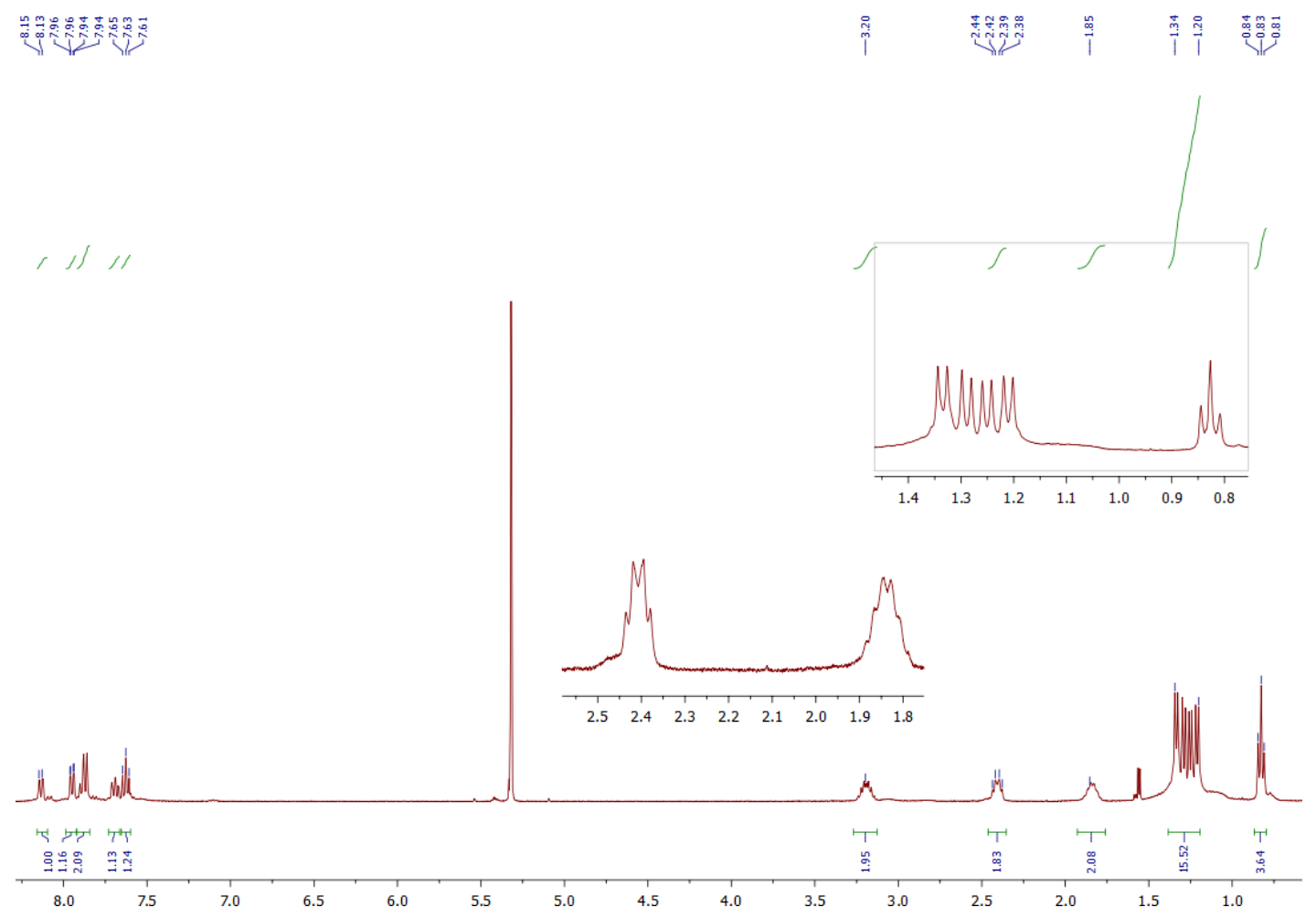


Figure S17. ${ }^{31} \mathrm{P}\left\{{ }^{1} \mathrm{H}\right\}$ NMR spectrum of complex $\mathbf{3 b}$

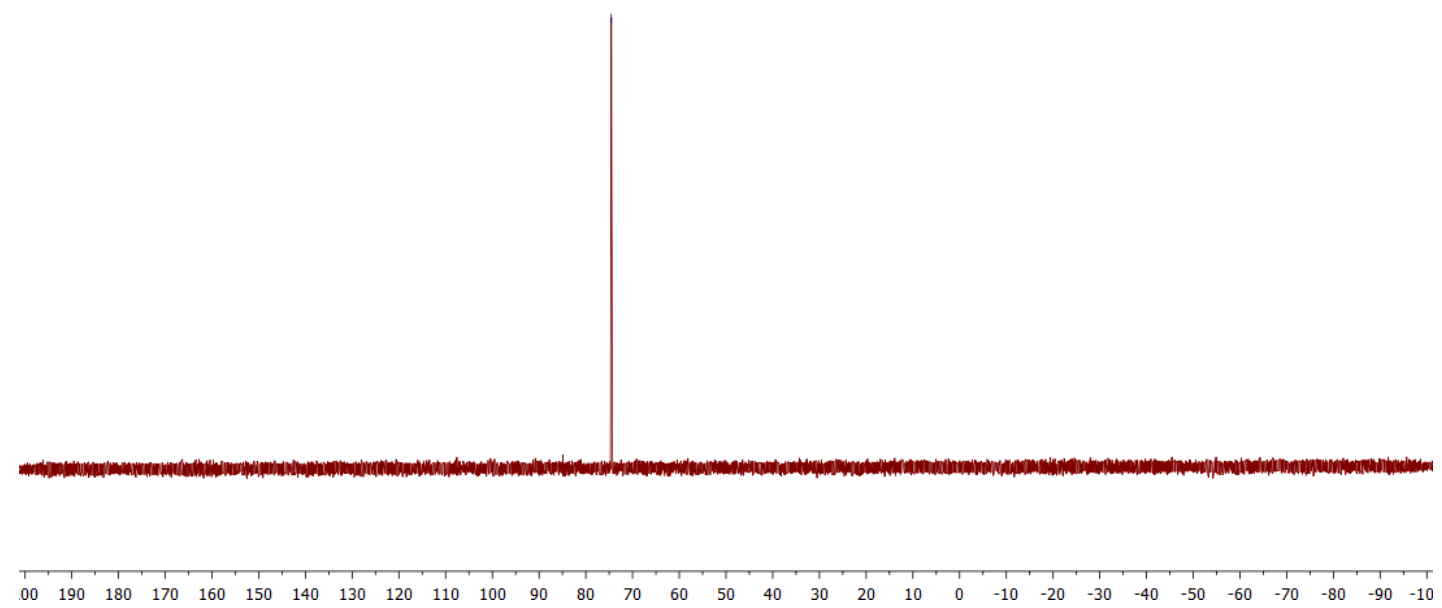

Figure S18. ${ }^{13} \mathrm{C}\left\{{ }^{1} \mathrm{H}\right\}$ NMR spectrum of complex $\mathbf{3 b}$

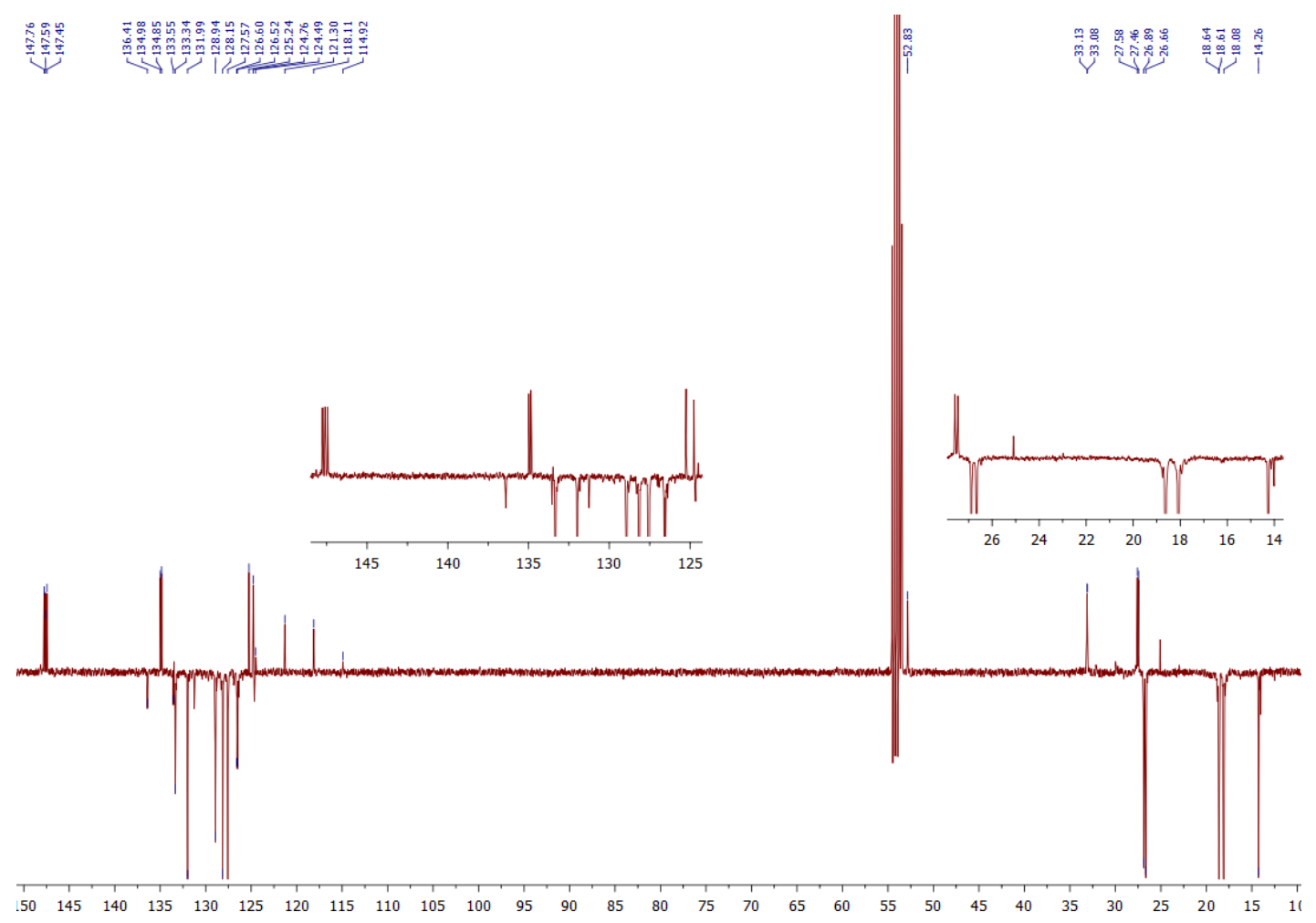


Figure S19. ${ }^{19} \mathrm{~F}\left\{{ }^{1} \mathrm{H}\right\}$ NMR spectrum of complex $\mathbf{3 b}$

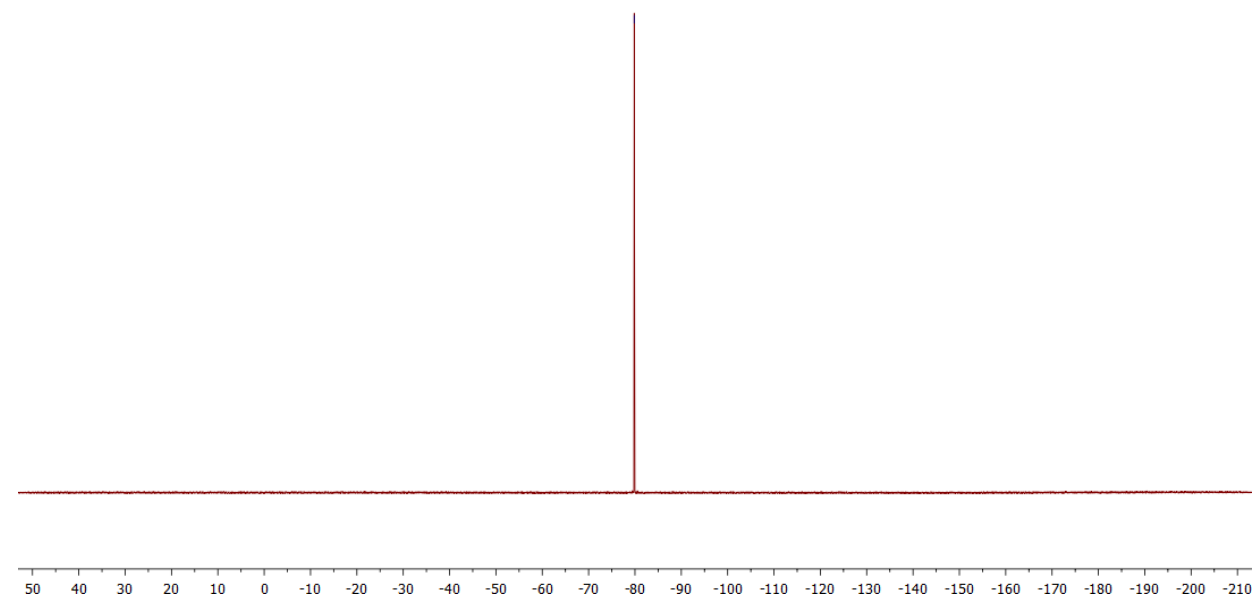

Figure S20. ${ }^{1} \mathrm{H}$ NMR spectrum of complex 4 with butane (*) and 2-butene (cis and trans)(arrows)

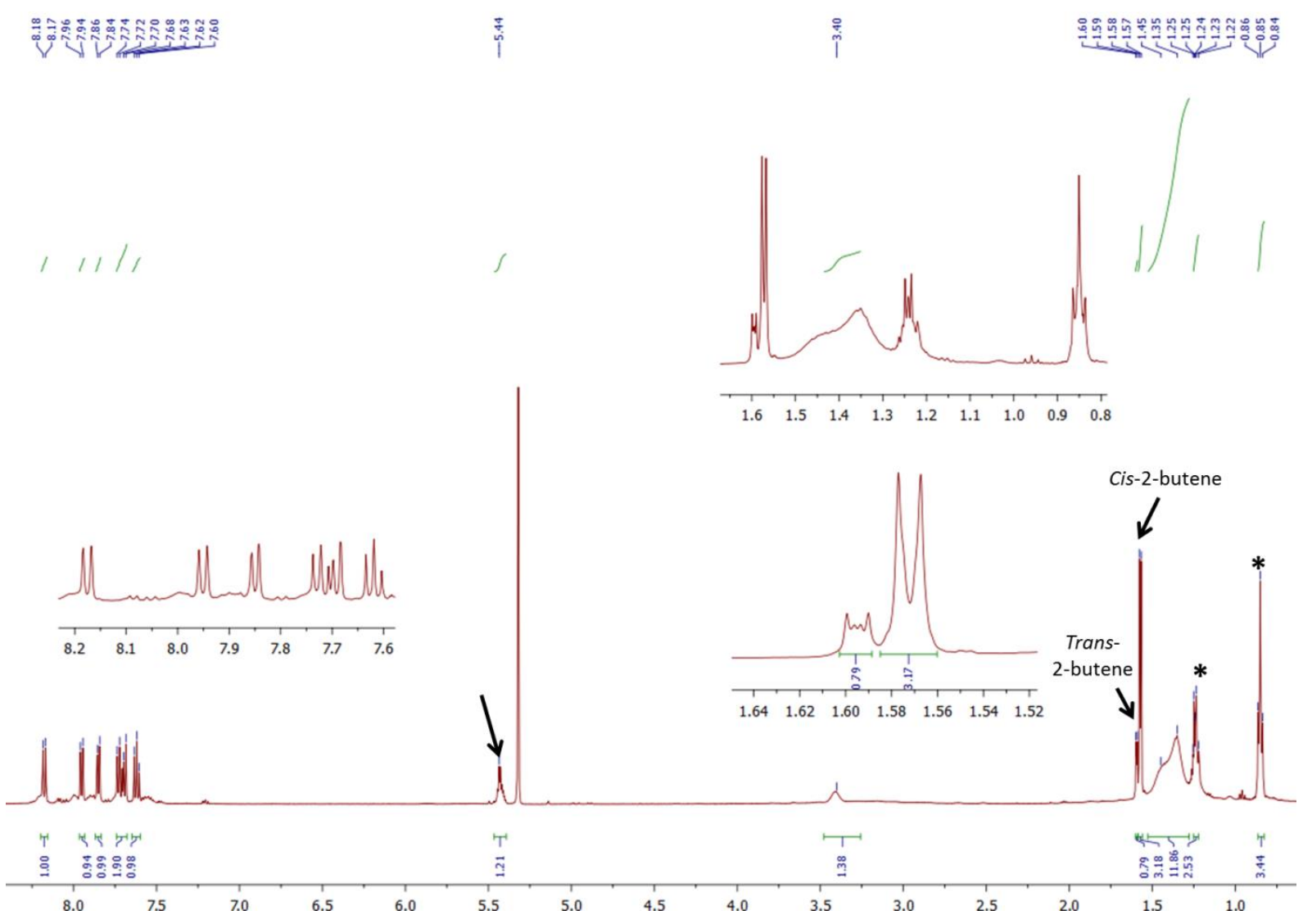


Figure S21. ${ }^{13} \mathrm{C}\left\{{ }^{1} \mathrm{H}\right\}$ NMR spectrum of complex 4 with butane $(*)$ and 2-butene (cis and trans) (arrows).
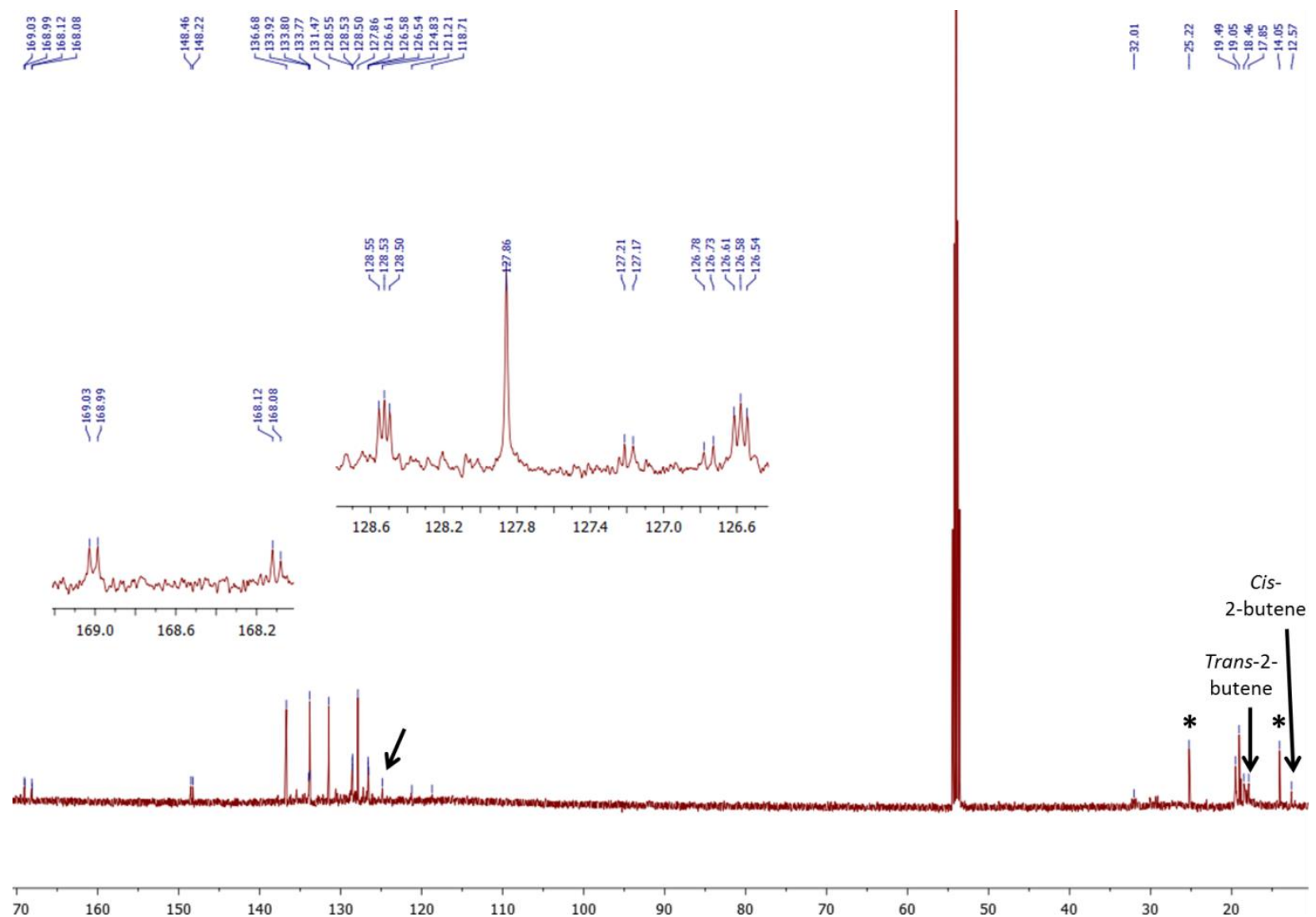

Figure S22. ${ }^{1} \mathrm{H}$ NMR spectrum of complex $3 \mathbf{c}$

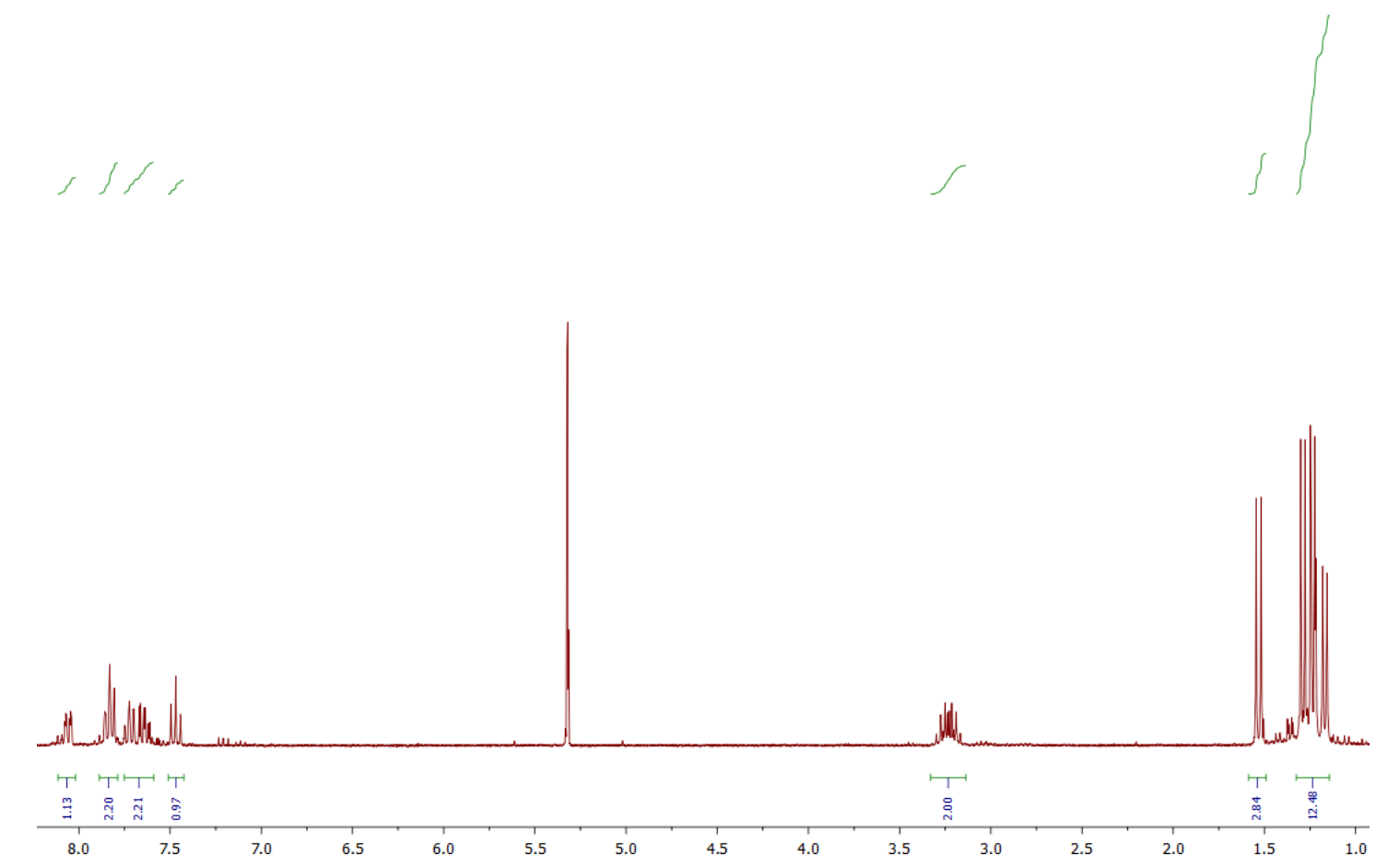


Figure S23. ${ }^{31} \mathrm{P}\left\{{ }^{1} \mathrm{H}\right\}$ NMR spectrum of complex 3c

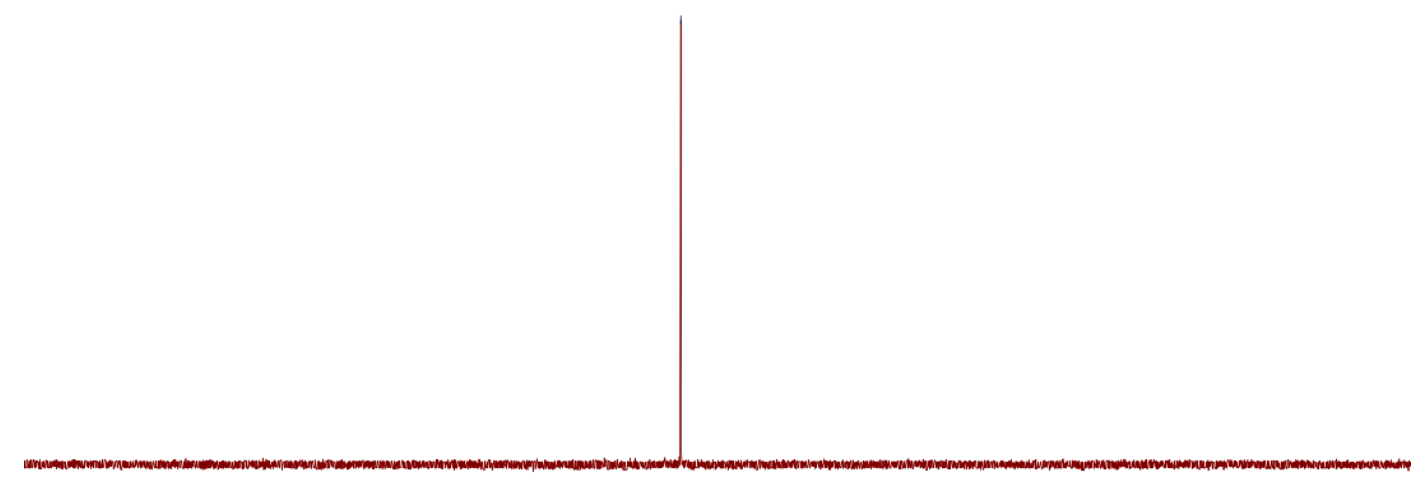

$\begin{array}{llllllllllllllllllllllllllllllllll}230 & 220 & 210 & 200 & 190 & 180 & 170 & 160 & 150 & 140 & 130 & 120 & 110 & 100 & 90 & 80 & 70 & 60 & 50 & 40 & 30 & 20 & 10 & 0 & -10 & -20 & -30 & -40 & -50 & -60 & -70 & -80 & -90 & -11\end{array}$

Figure S24. ${ }^{13} \mathrm{C}\left\{{ }^{1} \mathrm{H}\right\}$ NMR spectrum of complex $\mathbf{3 c}$

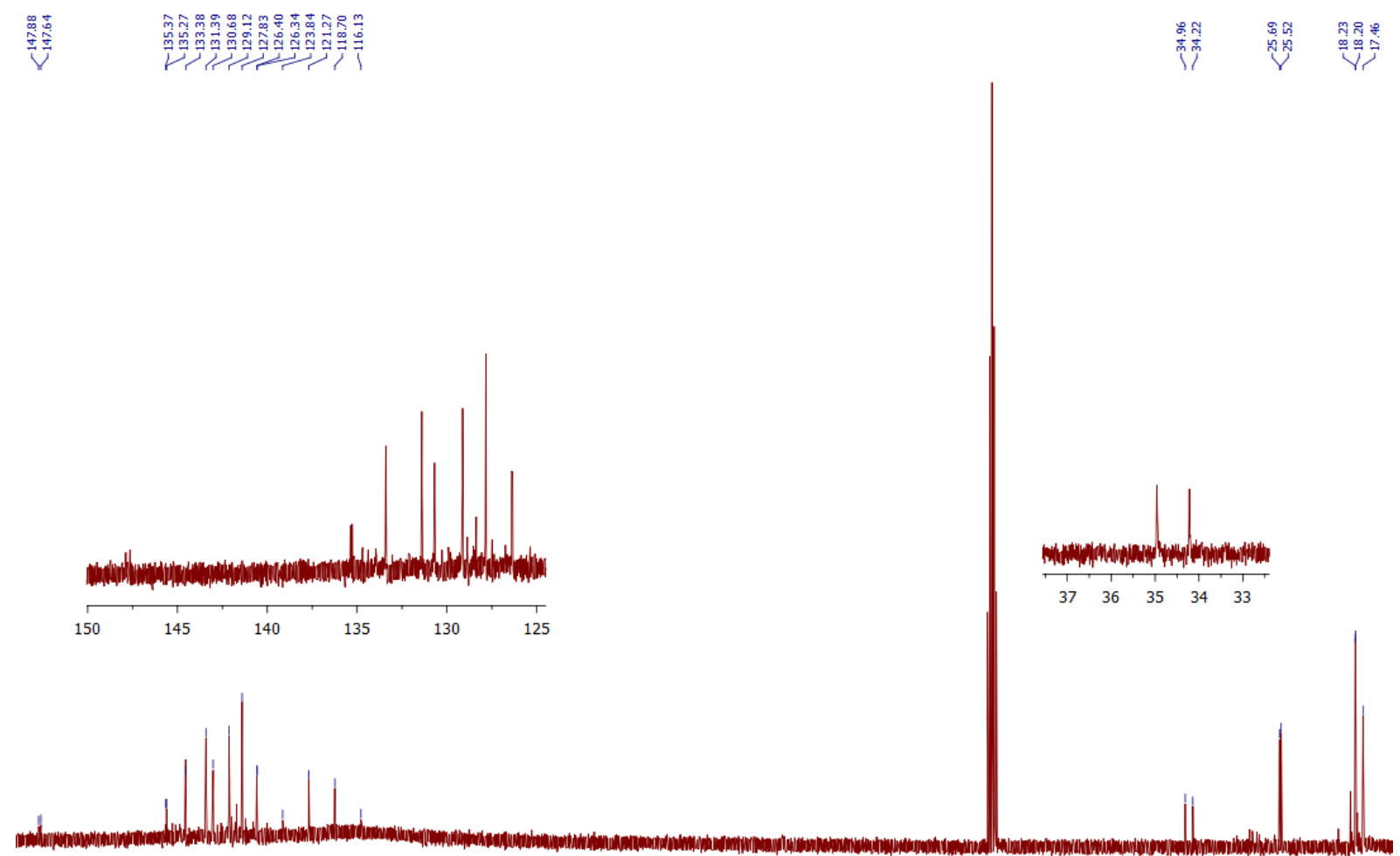

$\begin{array}{lllllllllllllllllllllllllllllllllllllllll}50 & 145 & 140 & 135 & 130 & 125 & 120 & 115 & 110 & 105 & 100 & 95 & 90 & 85 & 80 & 75 & 70 & 65 & 60 & 55 & 50 & 45 & 40 & 35 & 30 & 25 & 20 & 15\end{array}$ 
Figure S25. ${ }^{19} \mathrm{~F}$ NMR spectrum of complex 3c

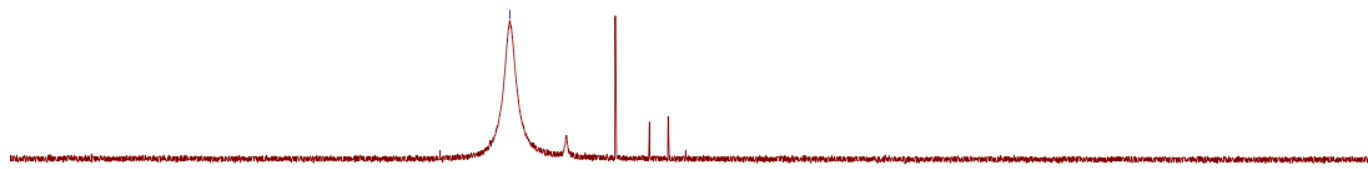


Figure S26. ${ }^{1} \mathrm{H}$ NMR spectrum of complex 5 with propylene (arrow), 2-butene ( $\bullet$ ) and 1-butene (*)

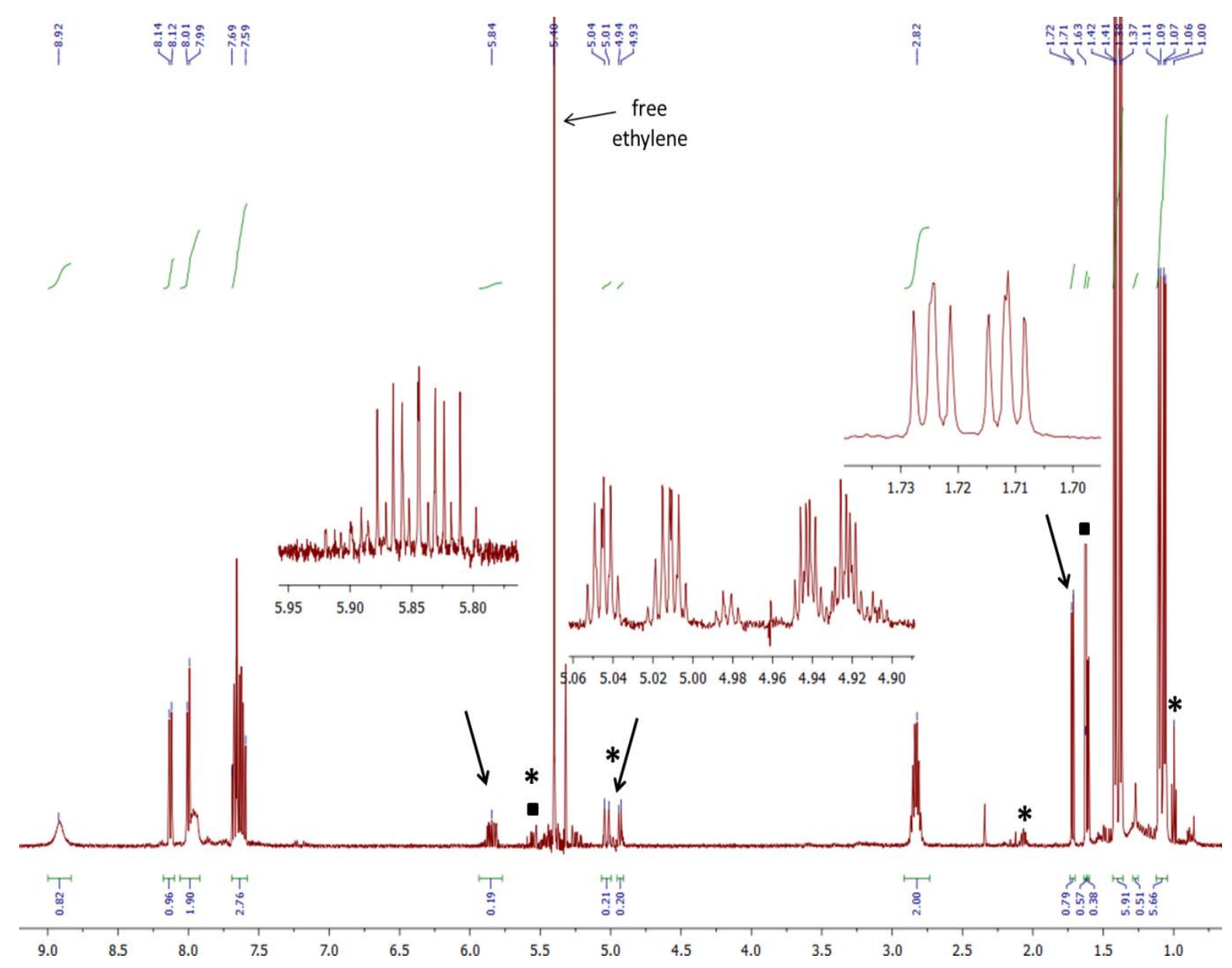


Figure S27. ${ }^{31} \mathrm{P}\left\{{ }^{1} \mathrm{H}\right\}$ NMR spectrum of complex 5
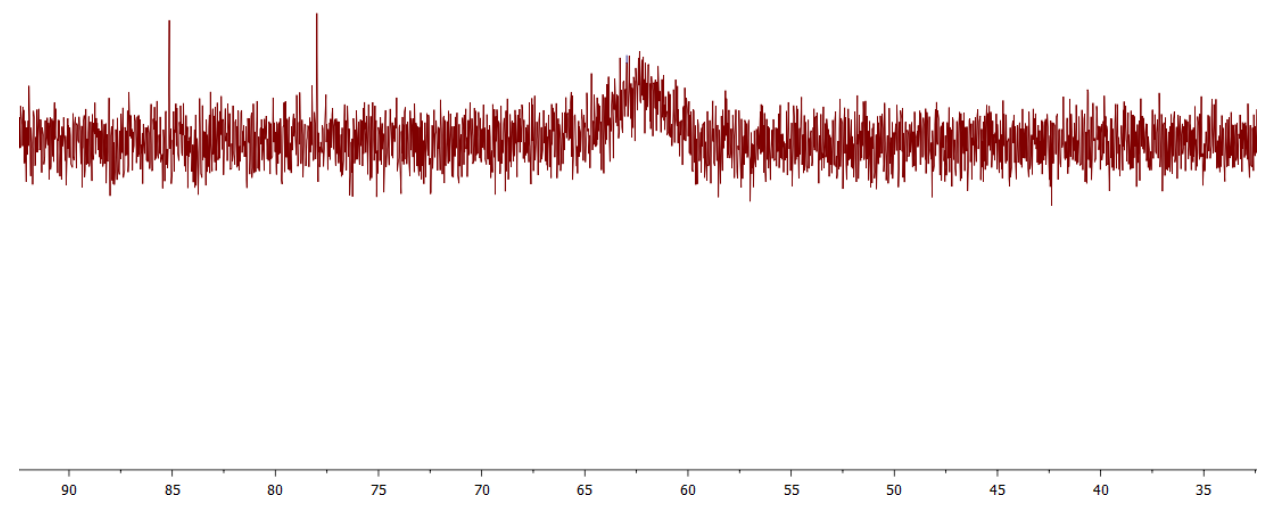

Figure S28. ${ }^{13} \mathrm{C}\left\{{ }^{1} \mathrm{H}\right\}$ NMR spectrum of complex 5 with propylene (arrow), 2-butene (a) and 1-butene

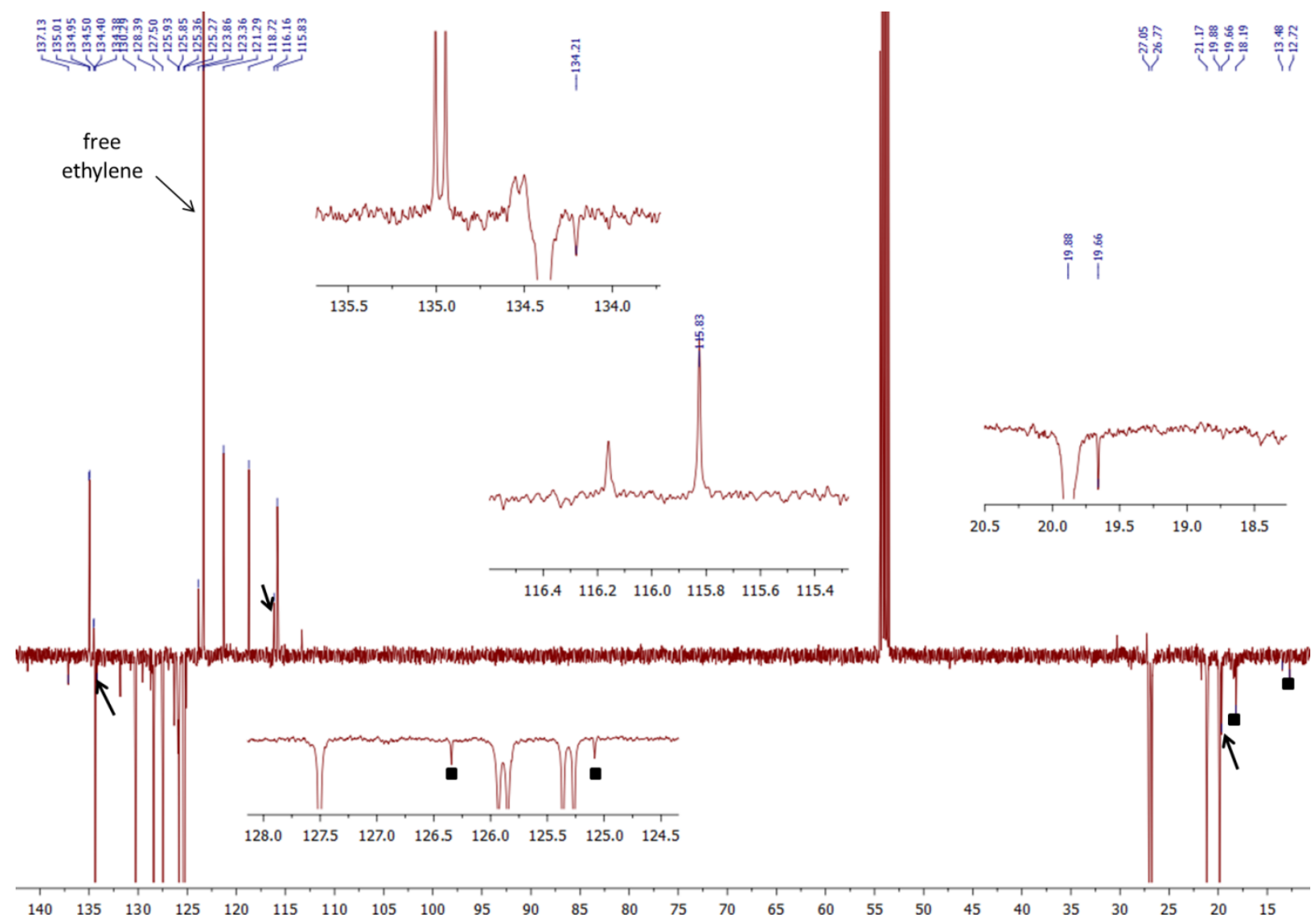


Figure S29. COSY NMR spectrum of complex 5 with propylene, 2-butene and 1-butene

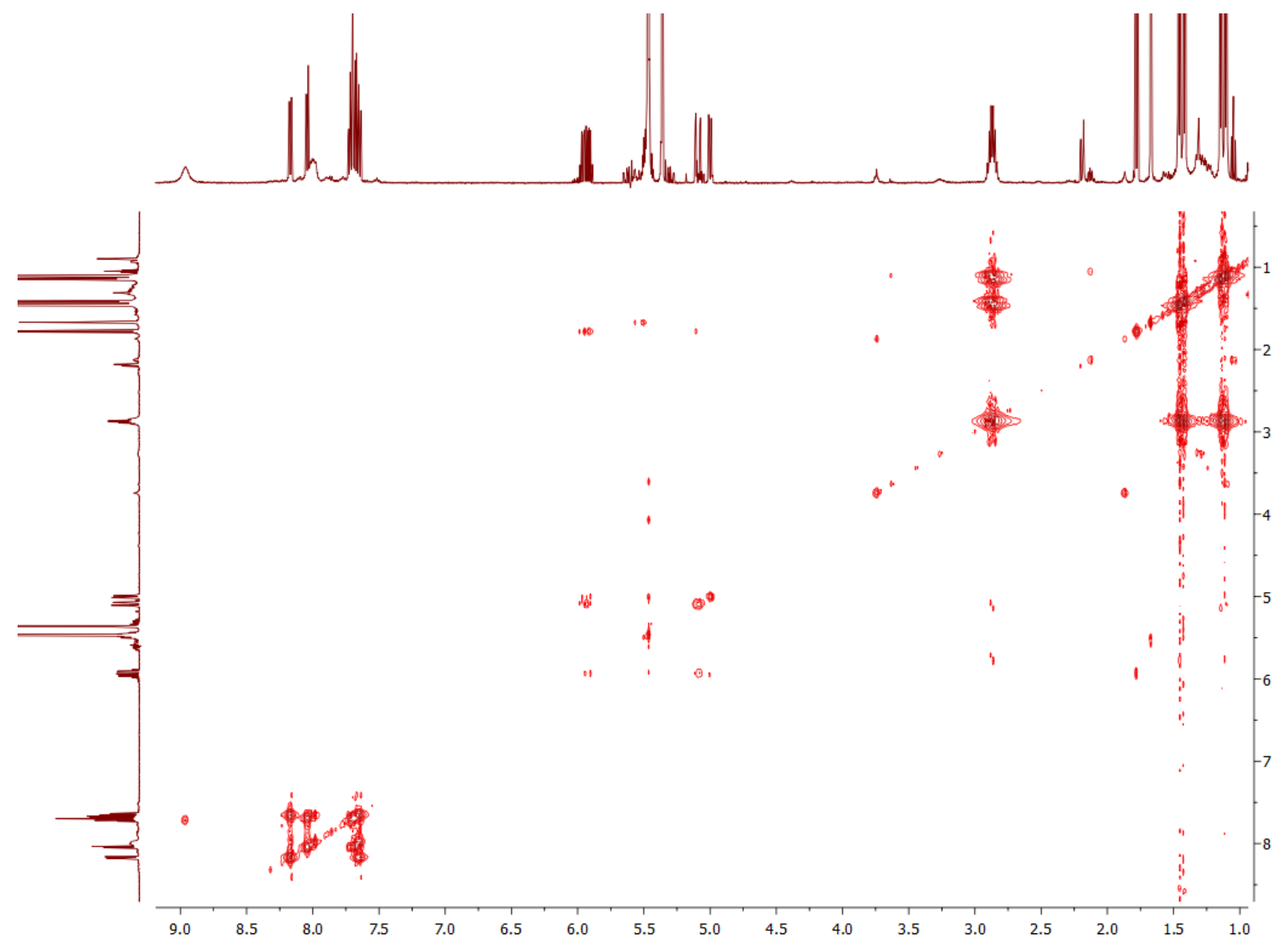

Figure S30. HSQC NMR spectrum of complex 5 with propylene, 2-butene and 1-butene

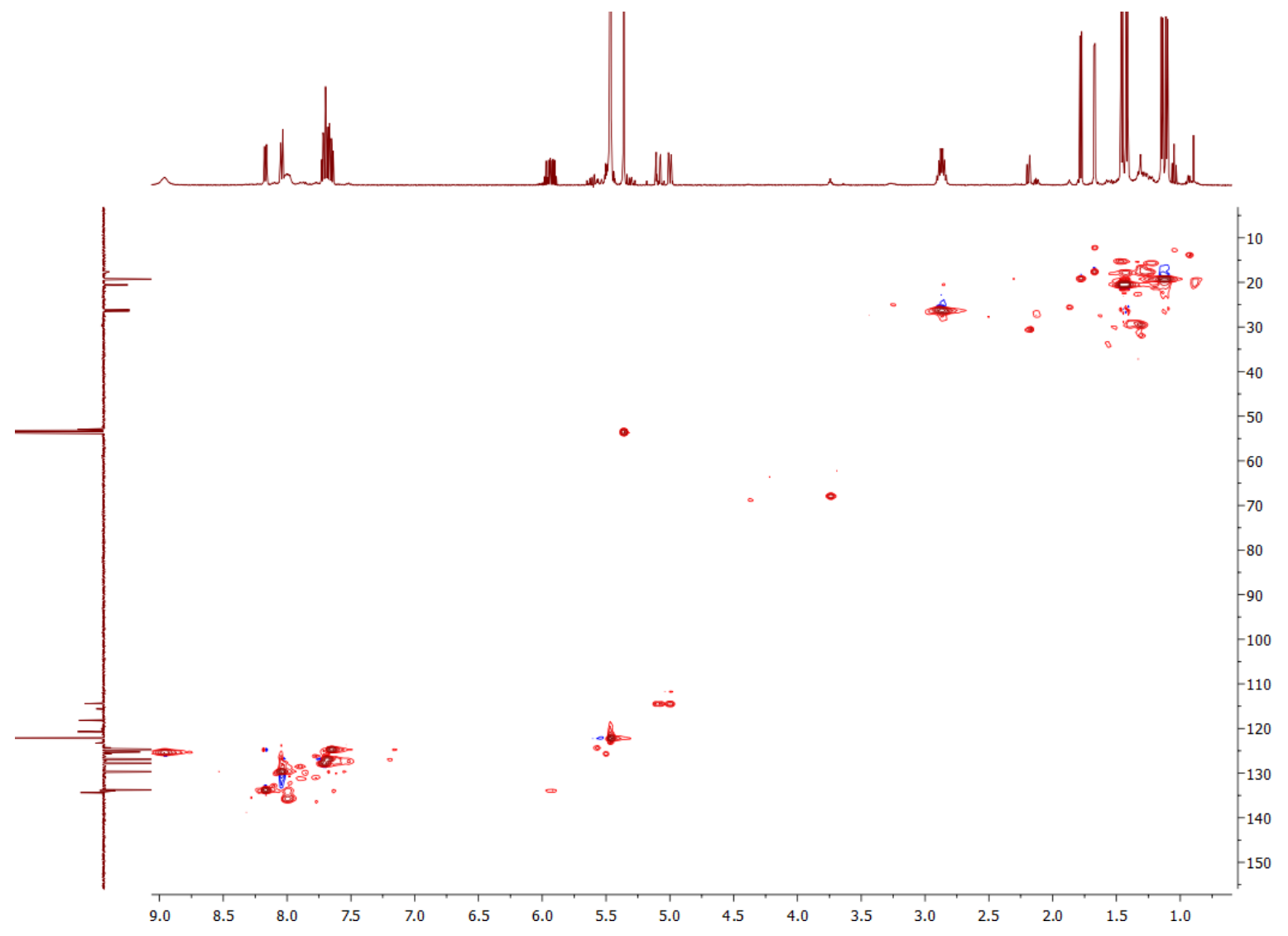


Figure S31. HMBC NMR spectrum of complex 5 with propylene, 2-butene and 1-butene

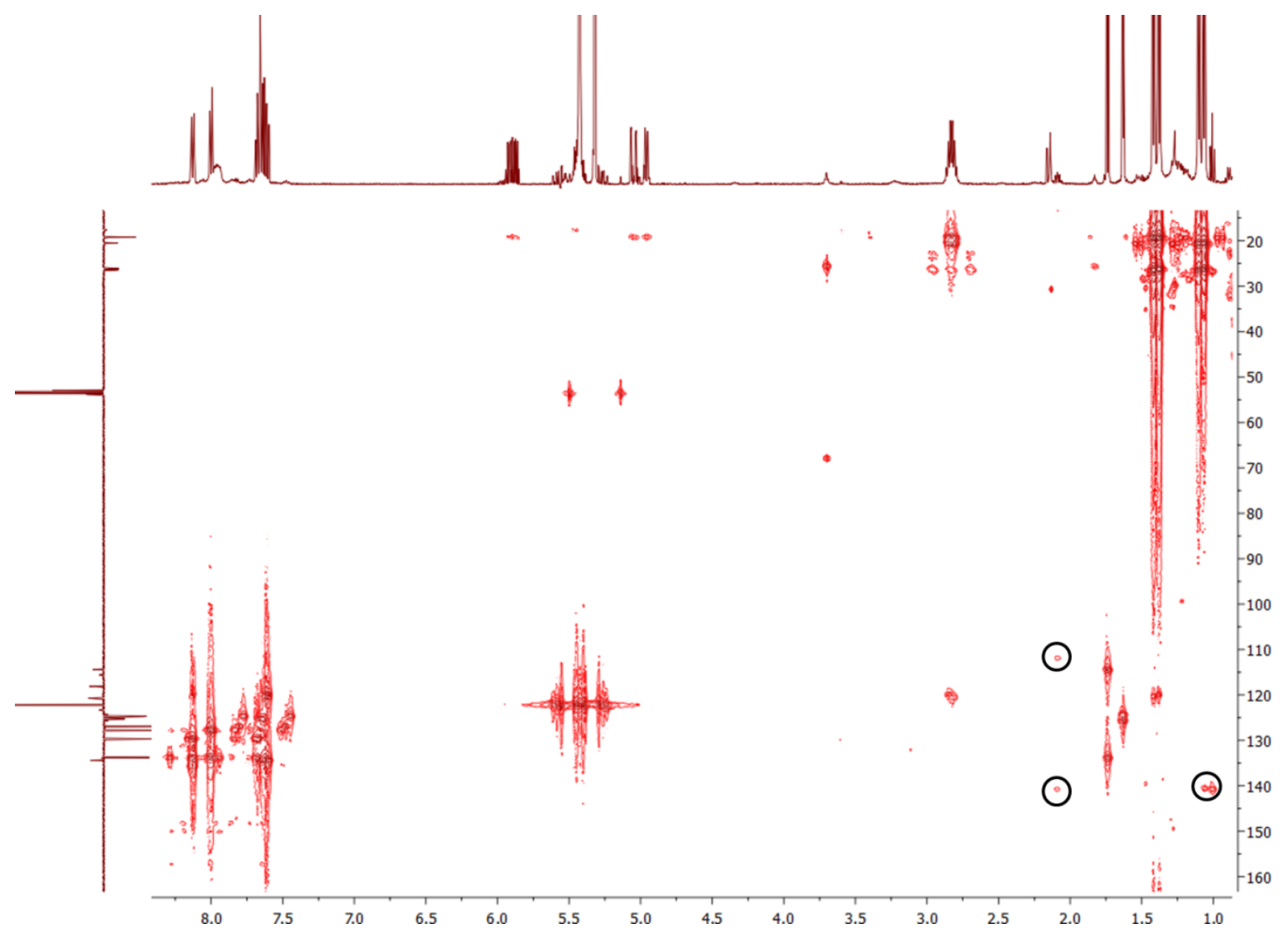




\section{Crystallographical data}

Crystallographic data were collected at 193(2) K on a Bruker-AXS Kappa APEX II Quazar diffractometer, with Mo $\mathrm{K}_{\alpha}$ radiation $(\lambda=0.71073 \AA$ ) using an oil-coated shock-cooled crystal. Phi- and omega-scans were used. Space groups were determined on the basis of systematic absences and intensity statistics. Semi-empirical absorption correction was employed. ${ }^{3}$ The structures were solved by direct methods (SHELXS-97), ${ }^{4}$ and refined using the least-squares method on $F^{2}$. All non-H atoms were refined with anisotropic displacement parameters. Hydrogen atoms were refined isotropically at calculated positions using a riding model with their isotropic displacement parameters constrained to be equal to 1.5 times the equivalent isotropic displacement parameters of their pivot atoms for terminal $\mathrm{sp}^{3}$ carbon and 1.2 times for all other atoms.

Crystallographic data have been deposited to the Cambridge Crystallographic Data Centre as supplementary publication. CCDC 1489288 (2a), 1489289 (2c). These data can be obtained free of charge from the Cambridge Crystallographic Data Centre via www.ccdc.cam.ac.uk/data_request/cif.

Figure S32. Molecular structure of complex 2c

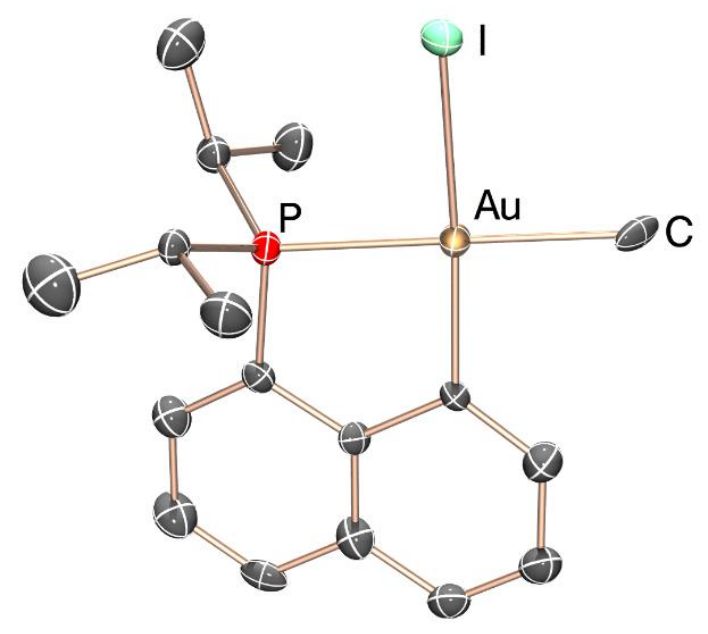

${ }^{3}$ Bruker, SADABS, Bruker AXS Inc., Madison, Wisconsin, USA, 2008.

${ }^{4}$ G. M. Sheldrick, Acta Cryst. A 2008, 64, 112-122. 
Table S1. Crystal Data, Data Collection, and Structure Refinement for 2a and 2c.

\begin{tabular}{|c|c|c|}
\hline & $2 \mathbf{a}$ & $2 c$ \\
\hline ID & Feriel33 & feriel_r \\
\hline formula & C19 H27 Au I P I & C17 H23 Au I \\
\hline$M_{r}$ & 610.24 & 582.19 \\
\hline crystal system & Monoclinic & Orthorhombic \\
\hline space group & $P 2{ }_{1}$ & $P b c a$ \\
\hline$a(\AA)$ & $8.5059(3)$ & $8.2625(5))$ \\
\hline$b(\AA)$ & $10.5556(3)$ & $13.7282(8)$ \\
\hline$c(\AA)$ & $11.4816(4)$ & $32.2865(19)$ \\
\hline$\alpha\left(^{\circ}\right)$ & 90 & 90 \\
\hline$\beta\left(^{\circ}\right)$ & $93.9911(16)$ & 90 \\
\hline$\gamma\left({ }^{\circ}\right)$ & 90 & 90 \\
\hline$V\left(\AA^{3}\right)$ & $1028.37(6)$ & $3662.2(4)$ \\
\hline$Z$ & 2 & 8 \\
\hline$\rho_{\text {calc }}\left(\mathrm{g} \mathrm{cm}^{-3}\right)$ & 1.971 & 2.112 \\
\hline$\mu\left(\mathrm{mm}^{-1}\right)$ & 8.727 & 9.797 \\
\hline$F(000)$ & 576 & 2176 \\
\hline crystal size $\left(\mathrm{mm}^{3}\right)$ & $0.200 \times 0.060 \times 0.040$ & $0.5 \times 0.5 \times 0.05$ \\
\hline$T / \mathrm{K}$ & 193(2) & $193(2)$ \\
\hline measd reflns & 42154 & 48936 \\
\hline Unique reflns (Rint) & $5100(0.0425)$ & $3712(0.0343)$ \\
\hline reflns used for refinement & 5100 & 3712 \\
\hline refined parameters & 204 & 186 \\
\hline GOF on $\mathrm{F}^{2}$ & 1.092 & 1.901 \\
\hline $\mathrm{R}_{1}^{\mathrm{a}}[\mathrm{I}>2 \sigma(\mathrm{I})]$ & 0.0436 & 0.0477 \\
\hline $\mathrm{wR}_{2}{ }^{\mathrm{b}}$ [all data] & 0.1165 & 0.2184 \\
\hline
\end{tabular}




\section{Computational Details}

All calculations were performed using the Gaussian 09 package $^{[5]}$ and the B3PW91 hybrid functional on the real experimental systems. ${ }^{[6]}$ The gold atom was described with the relativistic electron core potential SDD and associated basis set, ${ }^{[7]}$ augmented by a set of f-orbital polarization functions. ${ }^{[8]}$ The $6-31 G^{* *}$ basis set were employed for other atoms. All stationary points involved were fully optimized. Optimizations were carried out taking into account the counter-anion $\left[\mathrm{NTf}_{2}\right]$ and solvent effect ( $\mathrm{DCM}: \mathrm{CH}_{2} \mathrm{Cl}_{2}$ ) by means of the dielectric continuum standard SMD model. ${ }^{[9]}$ Frequency calculations were undertaken to confirm the nature of the stationary points, yielding one imaginary frequency for transition states (TS), corresponding to the expected process, and all of them positive for minima. The connectivity of the transition states and their adjacent minima was confirmed by intrinsic reaction coordinate (IRC) ${ }^{[10]}$ calculations. Natural Bond Orbital ${ }^{[11]}$ calculations (NBO, 5.9 version) $)^{[12]}$ have been carried on solvent-optimized geometries to analyze the bonding situation, in particular for the description of agostic interactions. Natural Localized Molecular Orbital (NLMO) were plotted with Molekel $4.3^{[13]}$ and all the geometrical structures with Gaussview 5.0. ${ }^{[14]}$

5 Gaussian 09, Revision C.01, M. J. Frisch, G. W. Trucks, H. B. Schlegel, G. E. Scuseria, M. A. Robb, J. R. Cheeseman, G. Scalmani, V. Barone, B. Mennucci, G. A. Petersson, H. Nakatsuji, M. Caricato, X. Li, H. P. Hratchian, A. F. Izmaylov, J. Bloino, G. Zheng, J. L. Sonnenberg, M. Hada, M. Ehara, K. Toyota, R. Fukuda, J. Hasegawa, M. Ishida, T. Nakajima, Y. Honda, O. Kitao, H. Nakai, T. Vreven, J. A. Montgomery, Jr., J. E. Peralta, F. Ogliaro, M. Bearpark, J. J. Heyd, E. Brothers, K. N. Kudin, V. N. Staroverov, T. Keith, R. Kobayashi, J. Normand, K. Raghavachari, A. Rendell, J. C. Burant, S. S. Iyengar, J. Tomasi, M. Cossi, N. Rega, J. M. Millam, M. Klene, J. E. Knox, J. B. Cross, V. Bakken, C. Adamo, J. Jaramillo, R. Gomperts, R. E. Stratmann, O. Yazyev, A. J. Austin, R. Cammi, C. Pomelli, J. W. Ochterski, R. L. Martin, K. Morokuma, V. G. Zakrzewski, G. A. Voth, P. Salvador, J. J. Dannenberg, S. Dapprich, A. D. Daniels, O. Farkas, J. B. Foresman, J. V. Ortiz, J. Cioslowski, and D. J. Fox, Gaussian, Inc., Wallingford CT, 2009.

6 (a) A. D. Becke J. Chem. Phys., 1993, 98, 5648; (b) J. P. Perdew, in Electronic Structure of Solids '91, Ed. P. Ziesche and H. Eschrig, Akademie Verlag, Berlin, 1991, 11-20.

7 Andrae, D. ; Häussermann, U.; Dolg, M.; Stoll, H.; Preuss, H. Theor. Chim. Acta 1990, 77, 123.

8 Ehlers, A. W.; Biihme, M.; Dapprich, S.; Gobbi, A.; Hijllwarth, A.; Jonas, V.; Kiihler, K. F.; Stegmann, R.; Veldkamp, A.; Frenking, G. Chem. Phys. Letters, 1993, 208, 111.

6 Marenich, A. V.; Cramer, C. J.; Truhlar, D. G. J. Phys. Chem. B 2009, 113, 6378.

10 (a) Fukui, K. Acc. Chem. Res., 1981, 14, 363; (b) Hratchian, H. P.; Schlegel, H. B. in Theory and Applications of Computational Chemistry: The First 40 Years, Ed. Dykstra, C. E.; Frenking, G.; Kim, K. S.; Scuseria, G. Elsevier, Amsterdam, 2005, 195.

$8 \quad$ (a) Reed, E.; Curtiss, L. A.; Weinhold, F. Chem. Rev. 1988, 88, 899. (b) Foster J. P.; Weinhold, F. J. Am. Chem. Soc. 1980, 102, 7211. (c) Reed A. E.; Weinhold, F. J. Chem. Phys. 1985, 83, 1736.

9 NBO 5.0 program, Glendening, E. D.; Badenhoop, J, K.; Reed, A. E.; Carpenter, J. E.; Bohmann, J. A.; Morales, C. M.; Weinhold, F. Theoretical Chemistry Institute, University of Wisconsin, Madison, 2001.

13 MOLEKEL 4.3, Flükiger, P.; Lüthi, H. P.; Portmann, S.; Weber, J. Swiss Center for Scientific Computing, Manno (Switzerland), 2000-2002.

14 GaussView, Version 5, Dennington, R.; Keith, T.; Millam, J.. Semichem Inc., Shawnee Mission, KS, 2009. 
Figure S33. Energy profile $\left(\Delta G(\Delta E)\right.$ values in $\left.\mathrm{kcal} \cdot \mathrm{mol}^{-1}\right)$ computed at B3PW91(SMD- $\left.\mathrm{CH}_{2} \mathrm{Cl}_{2}\right) / \mathrm{SDD}+\mathrm{f}(\mathrm{Au}) / 6-31 \mathrm{G}^{* *}$ (other atoms) level of theory for $\beta$-hydride elimination from [(P,C)gold(III)-butyl] $\left[\mathrm{NTf}_{2}\right]$ complex cis-3b, olefin rotation and 2,1 insertion of 1-butene into the Au-H bond. Formation of internal olefins (cis and trans 2-butene) has been considered for $\beta$-hydride elimination from cis-3' $\mathbf{b}_{\beta}$.

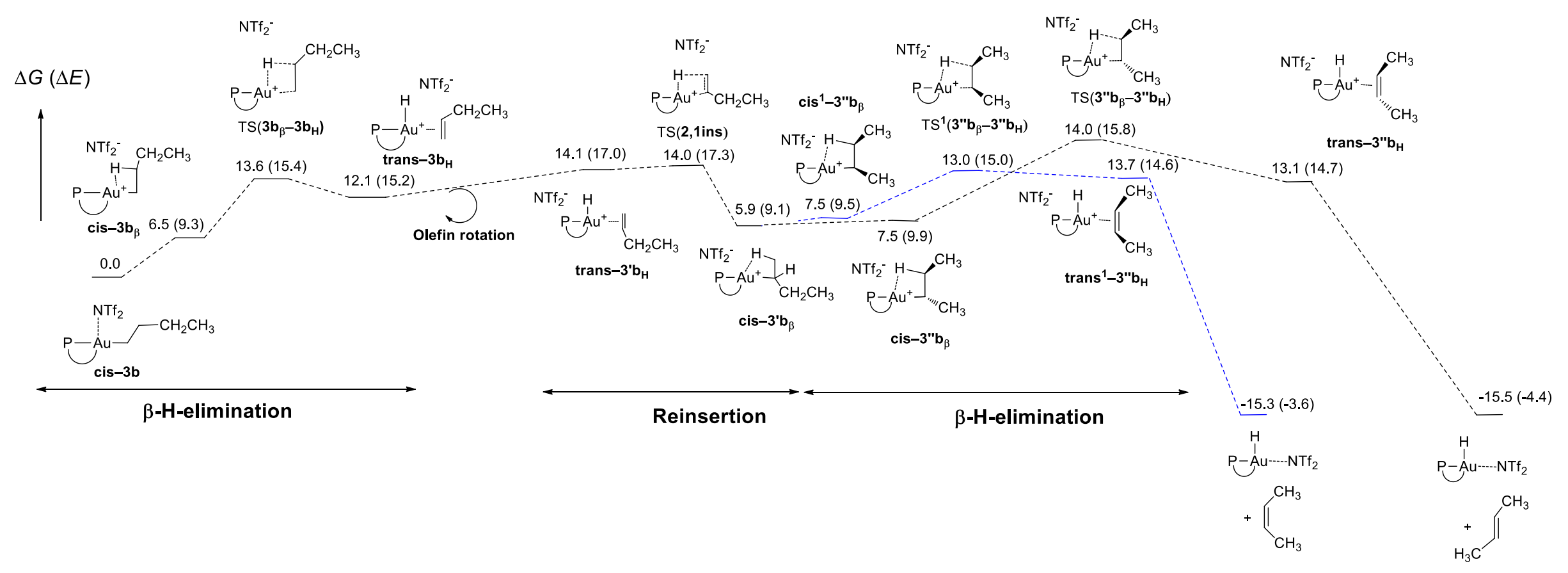

It is noteworthy that 1-butene decoordination from trans $\mathbf{- 3}^{\mathbf{\prime}} \mathbf{b}_{\mathbf{H}}$ is an exergonic process $\left(\Delta G(\Delta E)=-8.8\left(1.1 \mathrm{kcal} \cdot \mathrm{mol}^{-1}\right)\right)$. Nevertheless, this decoordination is much more favored from $\operatorname{trans}^{1}{ }^{1}-\mathbf{3}^{\prime}{ }^{\prime} \mathbf{b H}\left(\Delta G(\Delta E)=-15.8(-5.5) \mathrm{kcal} \cdot \mathrm{mol}^{-1}\right)$ or trans $-\mathbf{3}^{\prime}{ }^{\prime} \mathbf{b H}\left(\Delta G(\Delta E)=-16.5(-6.6) \mathrm{kcal} \cdot \mathrm{mol}^{-1}\right)$ than from trans-3'bH. 
Figure S34. NBO/NLMO analysis. Superposition of the donor $\sigma_{\mathrm{C}-\mathrm{H} \beta}$ and acceptor $\sigma^{*} \mathrm{C}-\mathrm{Au}$ (chicken-wire) NBOs (cutoff: $0.07 \mathrm{au}$ ) associated with the $\beta$-agostic interaction in complex cis$3 \mathbf{b}_{\beta}$. Stabilizing energy $\Delta \mathrm{E}(2)$ found at the second order perturbation theory is around 29.1 $\mathrm{kcal} / \mathrm{mol}$. Atomic contributions of main atoms in the corresponding $\sigma_{\mathrm{C}-\mathrm{H} \beta} \mathrm{NLMO}$ are shown as well. The counter-anion $\mathrm{NTf}_{2}$ is omitted for clarity.

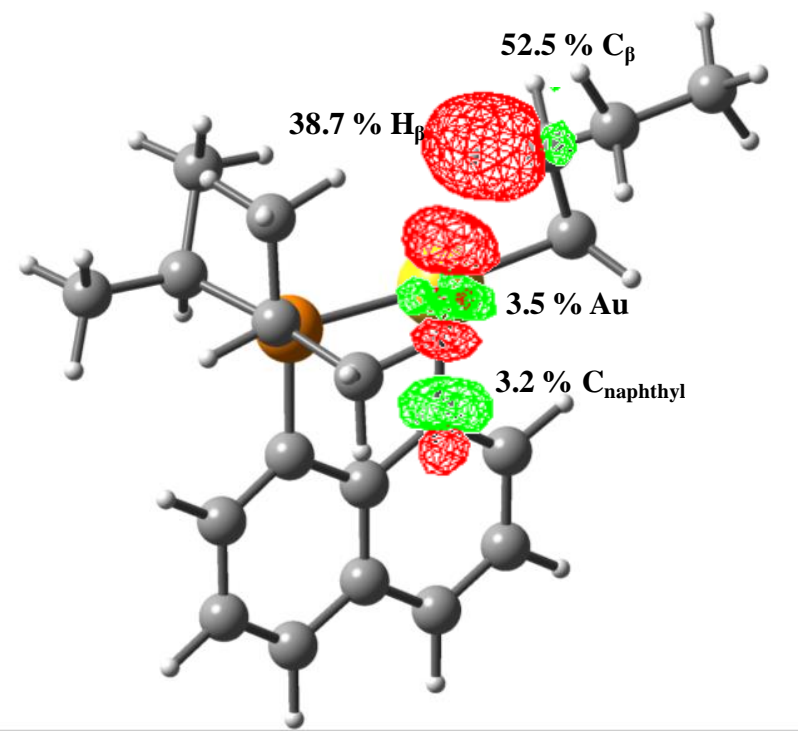


Figure S35. Relative energy stability (in $\mathrm{kcal} \cdot \mathrm{mol}^{-1}$ ) of the different isomers for eationic complex cis-3b found on the PES computed at the B3PW91( $\left.\mathrm{SMD}-\mathrm{CH}_{2} \mathrm{Cl}_{2}\right) / \mathrm{SDD}+\mathrm{f}(\mathrm{Au}), 6-$ $31 \mathrm{G}^{* *}$ (other atoms) level of theory. Main geometrical parameters with distances in $\AA$ and bond angles in $\left({ }^{\circ}\right)$. NBO analysis of the agostic interactions. The energy values into brackets correspond to those for the cationic complex, without taking into account counter-anion.

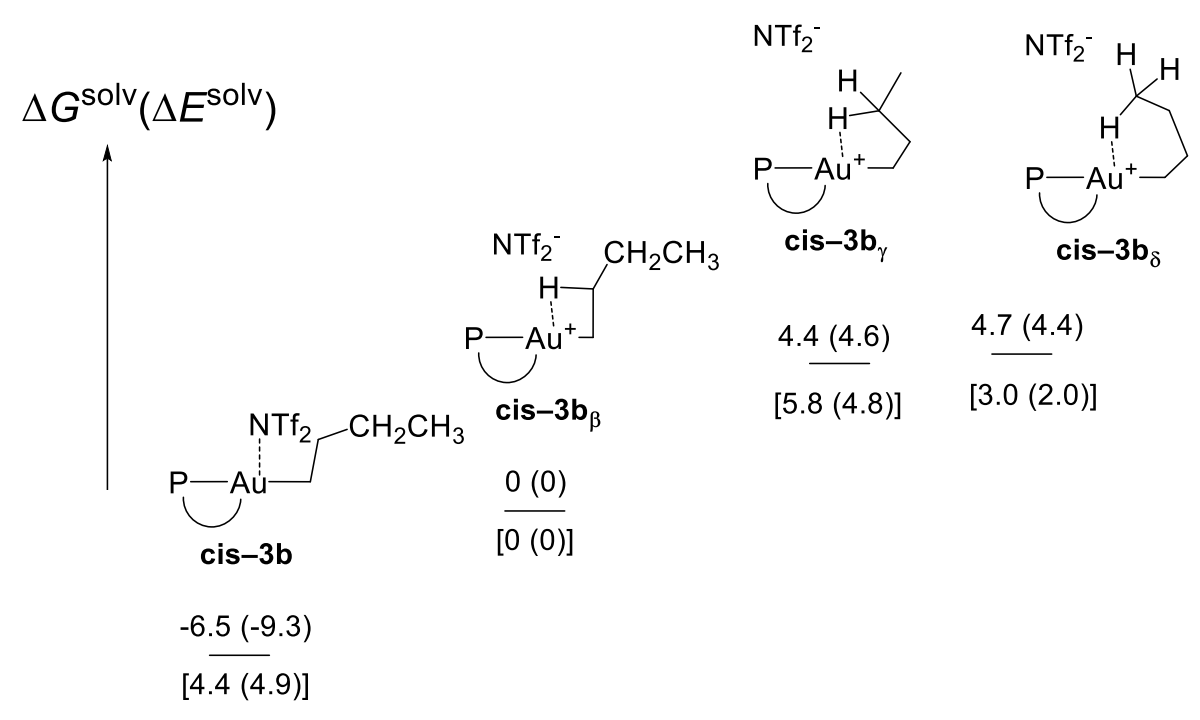

\begin{tabular}{|c|c|c|c|}
\hline & cis $-3 b_{\beta}$ & $\operatorname{cis}-3 b_{\gamma}$ & cis-3bs \\
\hline Au-N & 4.130 & 4.353 & 5.715 \\
\hline C-Hagostic & 1.184 & 1.128 & 1.135 \\
\hline Au-Hagostic & 1.954 & 2.158 & 1.956 \\
\hline C-HagosticAu & 99.0 & 115.6 & 118.2 \\
\hline$\sigma_{\mathrm{CH}} \rightarrow \sigma^{*}{ }_{\text {AuCnaphthyl }}{ }^{\mathrm{a}}$ & 29.1 & 12.1 & 24.6 \\
\hline
\end{tabular}

${ }^{\text {a }}$ Stabilizing energy $\Delta \mathrm{E}(2)$ in $\mathrm{kcal} \cdot \mathrm{mol}^{-1}$ found at the second order perturbation theory (NBO calculations). 
Figure S36. DFT-optimized structure of the butene hydride intermediate trans-3bH computed at the B3PW91(SMD- $\left.\mathrm{CH}_{2} \mathrm{Cl}_{2}\right) / \mathrm{SDD}+\mathrm{f}(\mathrm{Au}), 6-31 \mathrm{G}^{* *}$ (other atoms) level of theory. Selected bond distances $(\AA)$ and bond angles $\left({ }^{\circ}\right): \mathrm{AuH}: 1.615 ; \mathrm{AuC}_{\alpha}: 2.242 ; \mathrm{AuC}_{\beta}: 2.427 ; \mathrm{PAuC}_{\alpha}: 174.8$; $\mathrm{PAuC}_{\beta}$ : 150.4; PAuH: 86.88; $\mathrm{C}_{\text {naphthyl }} \mathrm{AuH}_{\beta}:$ 171.1; HAuC $\mathrm{C}_{\beta}$ : 6.1. The shortest Au-NTf 2 distances are $4.029 \AA(\mathrm{Au}-\mathrm{O})$ and $4.277 \AA(\mathrm{Au}-\mathrm{N})$.

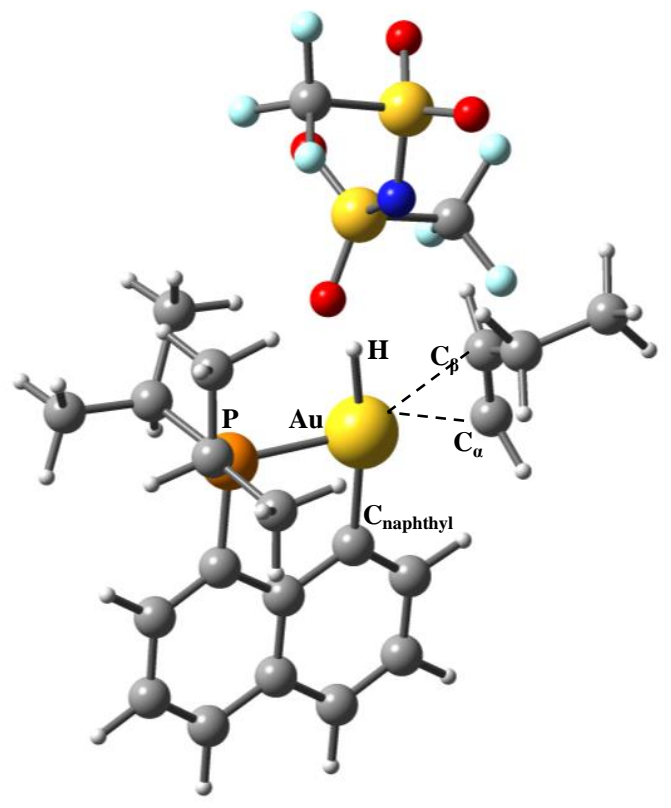

Figure S37. Olefin rotation $\left(\Delta G^{\mathrm{solv}}\left(\Delta E^{\mathrm{solv}}\right)\right.$ values in $\left.\mathrm{kcal} \cdot \mathrm{mol}^{-1}\right)$ from trans-3bH to trans-3' $\mathbf{b H}$ computed at the B3PW91(SMD- $\left.\mathrm{CH}_{2} \mathrm{Cl}_{2}\right) / \mathrm{SDD}+\mathrm{f}(\mathrm{Au}), 6-31 \mathrm{G} * *$ (other atoms) level of theory.

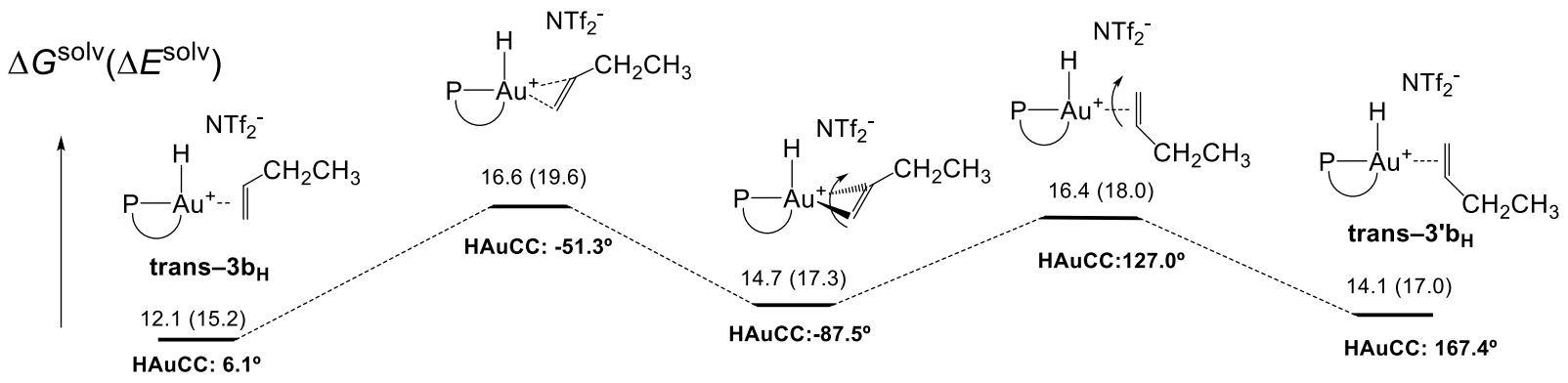


Figure S38. Energy profile $\left(\Delta G^{\text {solv }}\left(\Delta E^{\text {solv }}\right)\right.$ values in $\left.\mathrm{kcal} \cdot \mathrm{mol}^{-1}\right)$ for the $\beta$-hydride elimination from the trans isomer $[(\mathrm{P}, \mathrm{C})$ gold $(\mathrm{III})(n$-butyl $)]\left[\mathrm{NTf}{ }_{2}\right]$ complex trans $-3 \mathbf{b}_{\beta}$ computed at the B3PW91(SMD- $\left.\mathrm{CH}_{2} \mathrm{Cl}_{2}\right) / \mathrm{SDD}+\mathrm{f}(\mathrm{Au}), 6-31 \mathrm{G} * *$ (other atoms) level of theory. Formation of internal olefins (cis and trans $2-$ butenes) has been considered. Isomerization from $c i s-\mathbf{3} \mathbf{b}_{\beta}$ to trans $^{-} \mathbf{3} \mathbf{b}_{\beta}$ is also presented.

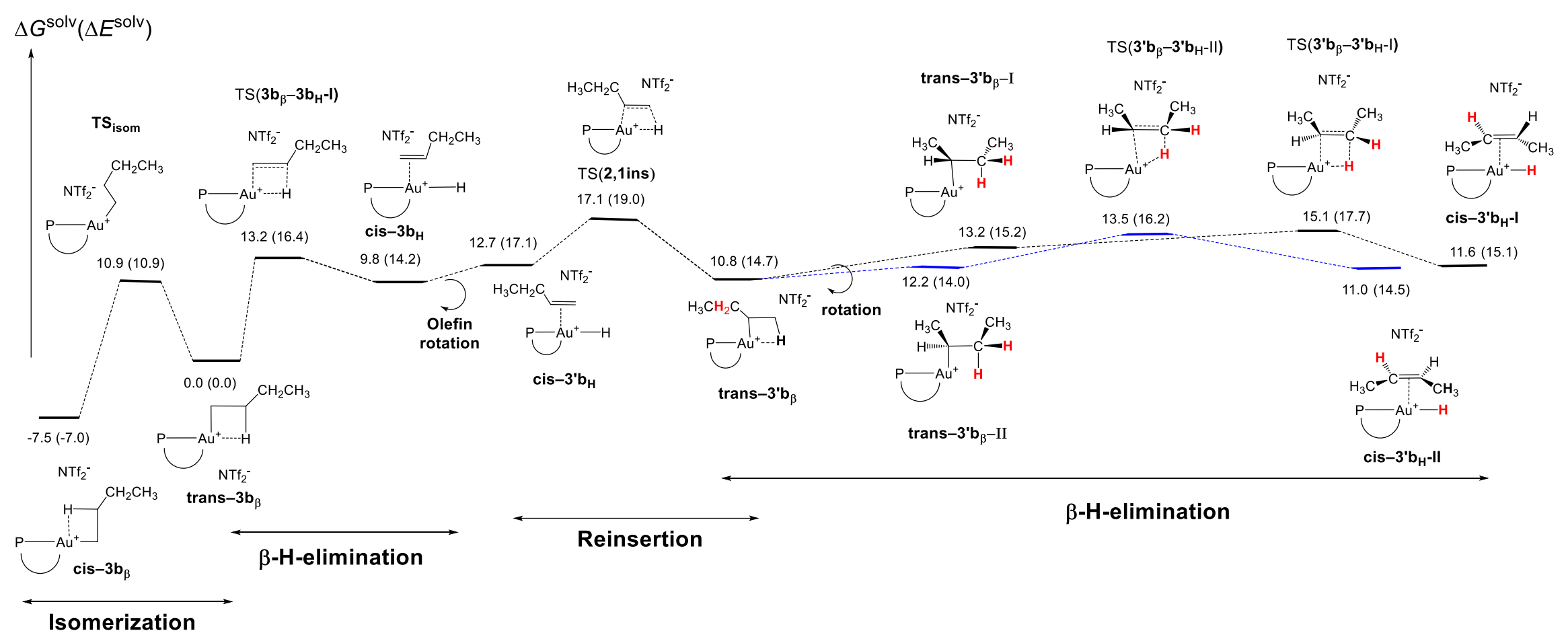


The $\mathrm{C}_{\mathrm{sp}}{ }^{2}-\mathrm{H}$ reductive elimination reaction has also been computed taking into account the [NTf 2$]$ counter-anion (figure S39). The reaction is also very favorable thermodynamically $\left(\Delta G=-39.6 \mathrm{kcal} \cdot \mathrm{mol}^{-1}\right)$. The associated activation barrier is $\left(\Delta G^{\neq}=25.7 \mathrm{kcal} \cdot \mathrm{mol}^{-1}\right.$. Considering the mild conditions in which the reaction occurs experimentally, it is more likely that the reductive elimination occurs directly from the 3-coordinate gold(III) species.

Figure S39. Reductive elimination step taking into account coordination by $\mathrm{NTf}_{2}$, computed at the B3PW91(SMD- $\left.\mathrm{CH}_{2} \mathrm{Cl}_{2}\right) / \mathrm{SDD}+\mathrm{f}(\mathrm{Au}), 6-31 \mathrm{G}^{* *}$ (other atoms) level of theory. Main geometrical parameters of the transition state (distances in $\AA$ and bond angles in ${ }^{\circ}$ ). The values into brackets correspond to those without taking into account $\mathrm{NTf}_{2}$ upon optimization in solvent.

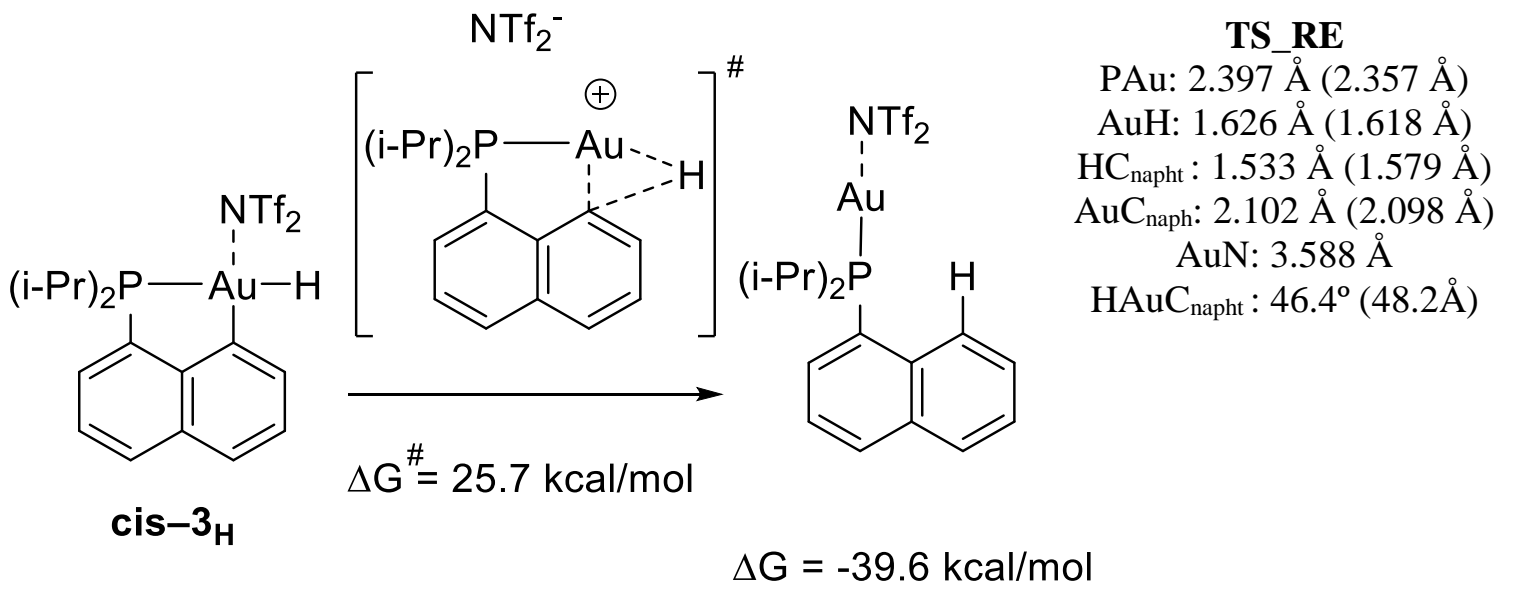


Cartesian coordinates.

\section{$\beta$-hydride elimination from [(P,C)gold(III)- butyl] $\left[\mathrm{NTf}_{2}\right]$ complex $c i s-3 \mathrm{~b}$}

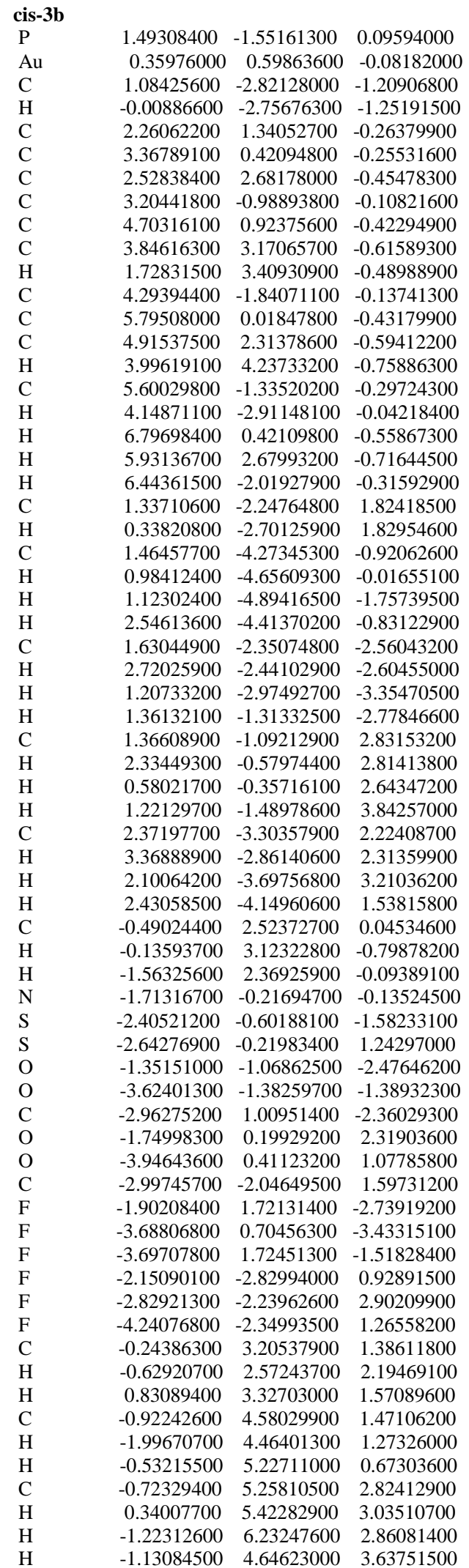

$\begin{array}{llll}-3.64509000 & -1.83830200 & -2.18601000\end{array}$

Sum of electronic and zero-point Energies= Sum of electronic and thermal Free Energies=
$-3082.697542$ $-3082.778554$ 


\section{$\operatorname{TS}(3 \mathrm{bb} \beta-3 \mathrm{bH})$}

$\mathrm{Au} \quad-1.21900600 \quad-0.08627500 \quad 0.85509300$

C $\quad-0.07965600 \quad-1.31703300 \quad 2.23251600$

$\begin{array}{llll}\mathrm{C} & 0.61715400 & -0.09754300 & 2.38517800\end{array}$

$\begin{array}{llll}\mathrm{H} & -0.12776000 & 1.07296800 & 1.25285900\end{array}$

$\begin{array}{llll}\mathrm{H} & 1.52890400 & 0.02454400 & 1.79917600\end{array}$

$\begin{array}{lllll}\mathrm{P} & -2.43623300 & 1.32262800 & -0.54282200\end{array}$

$\begin{array}{llll}\text { C } & -2.64510400 & -1.51768000 & 0.34511900\end{array}$

$\begin{array}{lllll}\text { C } & -3.76547300 & -1.08011300 & -0.43715000\end{array}$

$\begin{array}{llll}\text { C } & -2.60589800 & -2.84485600 & 0.73308300\end{array}$

$\begin{array}{llll}\text { C } & -3.87128400 & 0.26457900 & -0.90597900\end{array}$

C $\quad-4.82280900 \quad-1.99099300 \quad-0.76172800$

$\begin{array}{llll}\text { C } & -3.63597700 & -3.75508400 & 0.39202000\end{array}$

$\mathrm{H} \quad-1.77218500 \quad-3.22157100 \quad 1.31824100$

$\begin{array}{llll}\text { C } & -4.97973600 & 0.69149000 & -1.61302200\end{array}$

C $\quad-5.94023600-1.52201200-1.49940200$

C $\quad-4.72629700 \quad-3.33905700 \quad-0.33209300$

$\begin{array}{llll}\mathrm{H} & -3.55923500 & -4.78944900 & 0.71739400\end{array}$

$\begin{array}{llll}\text { C } & -6.02505200 & -0.21059100 & -1.90616200\end{array}$

$\mathrm{H} \quad-5.06161400 \quad 1.72162900 \quad-1.94632200$

$\mathrm{H} \quad-6.73860200 \quad-2.22184000 \quad-1.73408600$

$\mathrm{H} \quad-\quad-5.52659700 \quad-4.03013300 \quad-0.58458200$

$\begin{array}{llll}\mathrm{H} & -6.89132200 & 0.13740700 & -2.46123500\end{array}$

$\begin{array}{llll}\mathrm{C} & -3.05655600 & 2.85844600 & 0.28914900\end{array}$

$\mathrm{H} \quad-3.75478000 \quad 3.30431000 \quad-0.43215700$

$\begin{array}{llll}\mathrm{C} & -1.58674200 & 1.69414500 & -2.15968300\end{array}$

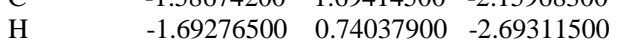

$\begin{array}{llll}\text { C } & -3.82439500 & 2.48371400 & 1.55901500\end{array}$

$\begin{array}{llll}\mathrm{H} & -3.16298800 & 2.01591300 & 2.29697000\end{array}$

$\begin{array}{llll}\mathrm{H} & -4.23986300 & 3.39103800 & 2.01052800\end{array}$

$\begin{array}{llll}\mathrm{H} & -4.65358100 & 1.79963200 & 1.35770300\end{array}$

$\begin{array}{lllll}\text { C } & -1.93476900 & 3.85427700 & 0.58354200\end{array}$

$\mathrm{H} \quad-1.42282800 \quad 4.19038000 \quad-0.32136900$

$\begin{array}{llll}\mathrm{H} & -2.35979200 & 4.73743100 & 1.07267200\end{array}$

$\mathrm{H} \quad-1.18814600 \quad 3.42515000 \quad 1.26112900$

$\begin{array}{llll}\text { C } & -0.09410100 & 1.97875300 & -1.98273400\end{array}$

$\mathrm{H} \quad 0.41560300 \quad 1.15460500 \quad-1.47809200$

$\mathrm{H} \quad 0.36127400 \quad 2.09829700 \quad-2.97173300$

$\begin{array}{llll}\mathrm{H} & 0.08583400 & 2.89823600 & -1.41968500\end{array}$

$\begin{array}{lllll}\text { C } & -2.29296200 & 2.78893800 & -2.96330700\end{array}$

$\begin{array}{llll}\mathrm{H} & -2.23650300 & 3.76075300 & -2.46358700\end{array}$

$\mathrm{H} \quad-1.79519100 \quad 2.88699700 \quad-3.93412100$

$\begin{array}{llll}\mathrm{H} & -3.34327900 & 2.55684900 & -3.15765000\end{array}$

$\begin{array}{llll}\mathrm{H} & 0.36598800 & -2.10094400 & 1.62568800\end{array}$

C $\quad 0.47788300 \quad 0.75708200 \quad 3.61763800$

$\begin{array}{llll}\mathrm{H} & -0.54521600 & 0.69435300 & 4.00332200\end{array}$

$\begin{array}{llll}\mathrm{H} & 0.68407600 & 1.80454700 & 3.37802300\end{array}$

$\begin{array}{llll}1.47088300 & 0.27065700 & 4.68046200\end{array}$

$\begin{array}{llll}\mathrm{H} & 1.26933600 & -0.76587200 & 4.96914600\end{array}$

$\begin{array}{llll}\mathrm{H} & 2.50156500 & 0.32941800 & 4.31685000\end{array}$

$\begin{array}{llll}\mathrm{H} & 1.39254000 & 0.89461200 & 5.57612200\end{array}$

$-0.75649000 \quad-1.63472700 \quad 3.02349900$

$\begin{array}{lll}3.10544300 & -0.28230100 & -0.09410300\end{array}$

$2.94410200 \quad-1.28482300 \quad-1.34860500$

$\begin{array}{llll}4.36397400 & 0.70345000 & 0.13374700\end{array}$

$\begin{array}{lll}1.51366200 & -1.56111700 & -1.51504100\end{array}$

$\begin{array}{lll}3.74598900 & -0.99065700 & -2.53693600\end{array}$

$3.63068800 \quad-2.88189100 \quad-0.68092400$

$\begin{array}{lll}4.45528100 & 0.98214900 & 1.56843900\end{array}$

$\begin{array}{llll}5.58676300 & 0.39473700 & -0.60818900\end{array}$

$\begin{array}{llll}3.74883600 & 2.30300000 & -0.59507000\end{array}$

$\begin{array}{lll}2.92383600 & -3.28376200 & 0.37840000\end{array}$

$3.55824300 \quad-3.82116600 \quad-1.62611200$

$4.90363100 \quad-2.73204800 \quad-0.31621500$

$\begin{array}{lll}2.68298400 & 2.74115000 & 0.08475900\end{array}$

$\begin{array}{llll}4.70955600 & 3.22540700 & -0.51945700\end{array}$

$\begin{array}{llll}3.40367300 & 2.14255300 & -1.87294500\end{array}$

Sum of electronic and zero-point Energies $=\quad-3082.687853$

Sum of electronic and thermal Free Energies $=\quad-3082.767236$

\section{trans-3bH}

$\begin{array}{llll}\mathrm{Au} & 1.14793700 & 0.08571400 & 0.80663200\end{array}$

$\begin{array}{llll}\text { C } & 0.07545000 & 1.56558100 & 2.10494800\end{array}$

$\begin{array}{llll}\text { C } & -0.61682800 & 0.41292800 & 2.44036400\end{array}$

$\mathrm{H} \quad 0.20886500-1.19511500 \quad 1.09976200$ $\begin{array}{lll}-1.52173300 & 0.18375500 & 1.87776800\end{array}$

$2.41377900-1.30744000 \quad-0.52188400$

$\begin{array}{lll}2.57192500 & 1.55377600 & 0.26247200\end{array}$

$\begin{array}{llll}3.74977800 & 1.08144800 & -0.41012300\end{array}$

$\begin{array}{llll}2.50096700 & 2.90788100 & 0.53778500\end{array}$

$\begin{array}{llll}3.88810200 & -0.28452600 & -0.79600800\end{array}$

$\begin{array}{llll}4.83085400 & 1.97204500 & -0.71261900\end{array}$

$\begin{array}{lll}3.55001900 & 3.80156600 & 0.20920800\end{array}$

$\begin{array}{lll}1.62779400 & 3.32788100 & 1.02708100\end{array}$

$\begin{array}{llll}5.04585800 & -0.75803200 & -1.38354600\end{array}$

$6.00487200 \quad 1.45722800 \quad-1.32116300$

$\begin{array}{llll}4.69969000 & 3.34546700 & -0.38722400\end{array}$

$\begin{array}{llll}3.44159700 & 4.85616400 & 0.44971800\end{array}$

$\begin{array}{llll}6.11871900 & 0.12314800 & -1.63733100\end{array}$

$\begin{array}{llll}5.14207500 & -1.80503500 & -1.65511400\end{array}$

$\begin{array}{lll}6.82285100 & 2.14185500 & -1.53227800\end{array}$

$\begin{array}{llll}5.51710900 & 4.02328600 & -0.61971100\end{array}$

$\begin{array}{llll}7.02740400 & -0.25847900 & -2.09348800\end{array}$

$\begin{array}{llll}2.95413800 & -2.87073100 & 0.30812300\end{array}$

$\begin{array}{llll}3.69526300 & -3.29412200 & -0.38421500\end{array}$

$\begin{array}{llll}1.62735100 & -1.61197400 & -2.18263000\end{array}$

$\begin{array}{llll}1.75462400 & -0.63581400 & -2.66924500\end{array}$

$\begin{array}{lll}3.64248000 & -2.54404100 & 1.63597900\end{array}$

$\begin{array}{lll}2.93471000 & -2.10680700 & 2.34895400\end{array}$

$\begin{array}{lll}4.03126000 & -3.46886700 & 2.07524200\end{array}$

$\begin{array}{llll}4.48104500 & -1.85269500 & 1.51274000\end{array}$

$\begin{array}{llll}1.81557600 & -3.87232500 & 0.49437300\end{array}$

$\begin{array}{llll}1.36120200 & -4.17323600 & -0.45241800\end{array}$

$\begin{array}{llll}2.21140400 & -4.77287800 & 0.97605900\end{array}$

$\begin{array}{llll}1.03006800 & -3.46555000 & 1.14032500\end{array}$

$\begin{array}{lll}0.12938800 & -1.90766000 & -2.09084600\end{array}$

$-0.40847500 \quad-1.10748400 \quad-1.57759100$

$-0.27412700 \quad-1.98370700 \quad-3.10634300$

$-0.07562700 \quad-2.85130100 \quad-1.57972100$

$\begin{array}{llll}2.38137600 & -2.66908600 & -2.99423900\end{array}$

$2.29509200 \quad-3.66349900 \quad-2.54570400$

$\begin{array}{llll}1.94132000 & -2.71830300 & -3.99596600\end{array}$

$\begin{array}{llll}3.44126700 & -2.42984800 & -3.11517500\end{array}$

$\begin{array}{lll}-0.34798900 & 2.25250100 & 1.37697500\end{array}$

$\begin{array}{lll}-0.38516300 & -0.34961800 & 3.71165300\end{array}$

$\begin{array}{lll}0.64429000 & -0.20555700 & 4.05665200\end{array}$

$\begin{array}{lll}-0.53940500 & -1.41949800 & 3.54271200\end{array}$

$\begin{array}{lll}-1.36944900 & 0.14247300 & 4.78264700\end{array}$

$\begin{array}{lll}-1.21474500 & 1.20268600 & 5.00709300\end{array}$

$\begin{array}{llll}-2.40685300 & 0.00983300 & 4.45996000\end{array}$

$\begin{array}{lll}-1.22799800 & -0.42585500 & 5.70718000\end{array}$

$\begin{array}{lll}0.83272000 & 1.95593900 & 2.78129000\end{array}$

$\begin{array}{llll}-3.02176900 & 0.25460100 & -0.12978600\end{array}$

$\begin{array}{llll}-3.01395200 & 1.18315400 & -1.44917500\end{array}$

$\begin{array}{llll}-4.20236500 & -0.77382000 & 0.25936300\end{array}$

$\begin{array}{llll}-1.61709600 & 1.50539000 & -1.75661700\end{array}$

$\begin{array}{lll}-3.90263200 & 0.78518200 & -2.54197900\end{array}$

$\begin{array}{llll}-3.71045600 & 2.79032200 & -0.81556400\end{array}$

$\begin{array}{lll}-4.15684900 & -0.97388400 & 1.70967600\end{array}$

$\begin{array}{llll}-5.49839000 & -0.56632900 & -0.38729400\end{array}$

$-3.57044200 \quad-2.38218000 \quad-0.43543800$

$\begin{array}{lll}-2.91822100 & 3.29580500 & 0.13340600\end{array}$

$-3.78515400 \quad 3.66429900-1.82118200$

$\begin{array}{llll}-4.92874600 & 2.61007000 & -0.30629100\end{array}$

$-2.44929000-2.75002700 \quad 0.19471800$

$-4.48819700 \quad-3.33435400-0.26006500$

$\begin{array}{lll}-3.30936500 & -2.26764100 & -1.73813200\end{array}$

Sum of electronic and zero-point Energies $=\quad-3082.688259$

Sum of electronic and thermal Free Energies $=\quad-3082.769717$

trans-3'bH

$\mathrm{Au} \quad-1.09927900 \quad-0.05996500 \quad 0.83290700$

$\begin{array}{llll}\text { C } & -0.16281200 & -1.79312300 & 2.08141200\end{array}$

$\begin{array}{llll}\text { C } & 0.60538900 & -0.66403600 & 2.29187600\end{array}$

$\begin{array}{llll}\mathrm{H} & -0.30439800 & 1.20640000 & 1.43396900\end{array}$

$\begin{array}{llll}\mathrm{H} & 1.48974600 & -0.47421700 & 1.68618700\end{array}$

$\begin{array}{lllll}\mathrm{P} & -2.36415400 & 1.52244600 & -0.26620600\end{array}$

$\begin{array}{llll}\text { C } & -2.38245400 & -1.44283000 & -0.14607700\end{array}$

C $\quad-3.55263000 \quad-0.88803800 \quad-0.76861000$

C $\quad-2.22266200 \quad-2.81537300 \quad-0.22462900$ 


\begin{tabular}{|c|c|c|c|}
\hline $\mathrm{C}$ & -3.76601300 & 0.52075200 & -0.83029500 \\
\hline $\mathrm{C}$ & -4.54555000 & -1.73501300 & -1.36099500 \\
\hline $\mathrm{C}$ & -3.17799400 & -3.65708000 & -0.84574500 \\
\hline $\mathrm{H}$ & -1.34737100 & -3.29569100 & 0.20007000 \\
\hline $\mathrm{C}$ & -4.92072400 & 1.05926600 & -1.36495700 \\
\hline $\mathrm{C}$ & -5.71970900 & -1.15362800 & -1.90617400 \\
\hline $\mathrm{C}$ & -4.32766600 & -3.13523500 & -1.38491700 \\
\hline $\mathrm{H}$ & -2.99729500 & -4.72855500 & -0.87682700 \\
\hline $\mathrm{C}$ & -5.91455800 & 0.20813000 & -1.89400500 \\
\hline $\mathrm{H}$ & -5.07309900 & 2.13429600 & -1.38755100 \\
\hline $\mathrm{H}$ & -6.47190300 & -1.81070500 & -2.33603000 \\
\hline $\mathrm{H}$ & -5.07692300 & -3.77883100 & -1.83886600 \\
\hline $\mathrm{H}$ & -6.82283700 & 0.63774400 & -2.30617600 \\
\hline $\mathrm{C}$ & -3.01019500 & 2.87016600 & 0.82162600 \\
\hline $\mathrm{H}$ & -3.73233800 & 3.39423500 & 0.17977100 \\
\hline $\mathrm{C}$ & -1.51116000 & 2.17939800 & -1.78454100 \\
\hline $\mathrm{H}$ & -1.56894000 & 1.31676800 & -2.46118000 \\
\hline $\mathrm{C}$ & -3.74863500 & 2.26926200 & 2.02000200 \\
\hline $\mathrm{H}$ & -3.05674700 & 1.73760700 & 2.68249200 \\
\hline $\mathrm{H}$ & -4.20923400 & 3.07723400 & 2.59819000 \\
\hline $\mathrm{H}$ & -4.54087500 & 1.57767000 & 1.71955700 \\
\hline $\mathrm{C}$ & -1.92850500 & 3.85611700 & 1.25985500 \\
\hline $\mathrm{H}$ & -1.44882700 & 4.35578000 & 0.41517300 \\
\hline $\mathrm{H}$ & -2.38720300 & 4.62782400 & 1.88750700 \\
\hline $\mathrm{H}$ & -1.15288600 & 3.35960300 & 1.85335900 \\
\hline $\mathrm{C}$ & -0.03510000 & 2.50489300 & -1.55064900 \\
\hline $\mathrm{H}$ & 0.51435500 & 1.63462400 & -1.18591100 \\
\hline $\mathrm{H}$ & 0.41097800 & 2.80602800 & -2.50450400 \\
\hline $\mathrm{H}$ & 0.10237500 & 3.32767300 & -0.84436800 \\
\hline $\mathrm{C}$ & -2.27612100 & 3.34728200 & -2.41332200 \\
\hline $\mathrm{H}$ & -2.25403800 & 4.23887200 & -1.77921200 \\
\hline $\mathrm{H}$ & -1.79648300 & 3.60521800 & -3.36348700 \\
\hline $\mathrm{H}$ & -3.31806000 & 3.09693100 & -2.63142100 \\
\hline $\mathrm{H}$ & 0.48705200 & -0.08126200 & 3.20198900 \\
\hline $\mathrm{H}$ & 0.16604400 & -2.47701200 & 1.30085200 \\
\hline $\mathrm{C}$ & -1.13348100 & -2.32934200 & 3.09941100 \\
\hline $\mathrm{H}$ & -2.00801600 & -2.75404800 & 2.59700300 \\
\hline $\mathrm{H}$ & -1.48482800 & -1.51311600 & 3.74099800 \\
\hline $\mathrm{C}$ & -0.46385800 & -3.41332200 & 3.95322300 \\
\hline $\mathrm{H}$ & 0.39585400 & -3.01193000 & 4.49961100 \\
\hline $\mathrm{H}$ & -1.17429000 & -3.81566300 & 4.68247600 \\
\hline $\mathrm{H}$ & -0.11133200 & -4.24535800 & 3.33422600 \\
\hline $\mathrm{N}$ & 3.10437100 & -0.17205100 & -0.04914900 \\
\hline S & 3.04407600 & -0.83328600 & -1.51841800 \\
\hline $\mathrm{O}$ & 1.63172100 & -1.01790200 & -1.86734000 \\
\hline $\mathrm{O}$ & 3.95248900 & -0.28922000 & -2.52854500 \\
\hline $\mathrm{C}$ & 3.65339100 & -2.56239200 & -1.19646600 \\
\hline $\mathrm{F}$ & 2.85154400 & -3.18276600 & -0.32698300 \\
\hline $\mathrm{F}$ & 3.65775700 & -3.24937800 & -2.34044800 \\
\hline $\mathrm{F}$ & 4.89010800 & -2.53934100 & -0.69962800 \\
\hline S & 4.32172700 & 0.72536400 & 0.51225900 \\
\hline $\mathrm{O}$ & 4.32268500 & 0.61406500 & 1.97221100 \\
\hline $\mathrm{O}$ & 5.59233600 & 0.63850500 & -0.20790000 \\
\hline $\mathrm{C}$ & 3.70381200 & 2.45176800 & 0.19230200 \\
\hline $\mathrm{F}$ & 2.59902000 & 2.69004700 & 0.90757200 \\
\hline $\mathrm{F}$ & 4.63976000 & 3.33315000 & 0.54855600 \\
\hline $\mathrm{F}$ & 3.41992000 & 2.62098300 & -1.10009500 \\
\hline
\end{tabular}

Sum of electronic and zero-point Energies $=\quad-3082.685386$ Sum of electronic and thermal Free Energies $=\quad-3082.766502$

$\begin{array}{lrrr}\text { TS(2-1ins) } & & & \\ \mathrm{Au} & 1.12706900 & -0.04995000 & 0.96061400 \\ \mathrm{C} & -0.01087100 & 1.27179400 & 2.33874800 \\ \mathrm{C} & -0.51442900 & -0.01735000 & 2.59957800 \\ \mathrm{H} & 0.37908300 & -1.29478700 & 1.71251000 \\ \mathrm{H} & -1.44374700 & -0.34305700 & 2.13765500 \\ \mathrm{P} & 2.43146400 & -1.50374100 & -0.30783100 \\ \mathrm{C} & 2.17030900 & 1.45365200 & -0.05376100 \\ \mathrm{C} & 3.28925300 & 1.04386700 & -0.85339800 \\ \mathrm{C} & 1.88672000 & 2.80618800 & 0.00705700 \\ \mathrm{C} & 3.62170900 & -0.33416100 & -1.02530300 \\ \mathrm{C} & 4.11325600 & 2.01732900 & -1.50666400 \\ \mathrm{C} & 2.67666500 & 3.77084900 & -0.66494000 \\ \mathrm{H} & 1.03881500 & 3.16335900 & 0.58338700 \\ \mathrm{C} & 4.73763000 & -0.72087400 & -1.74291600\end{array}$

$\begin{array}{llll}5.24620900 & 1.58590900 & -2.24391900\end{array}$

$\begin{array}{llll}3.77637200 & 3.39008100 & -1.39413600\end{array}$

$2.40996100 \quad 4.82185900 \quad-0.58747800$

$\begin{array}{llll}5.56236100 & 0.25110800 & -2.34887900\end{array}$

$\begin{array}{llll}4.99097400 & -1.77171200 & -1.84729200\end{array}$

$\begin{array}{llll}5.86854400 & 2.33641900 & -2.72547000\end{array}$

$\begin{array}{llll}4.39858500 & 4.12768800 & -1.89470700\end{array}$

$\begin{array}{llll}6.43859300 & -0.06376800 & -2.90797200\end{array}$

$\begin{array}{llll}3.36987800 & -2.75410900 & 0.68799200\end{array}$

$\begin{array}{llll}4.06163300 & -3.21795400 & -0.02866300\end{array}$

$\begin{array}{lll}1.49921000 & -2.28631700 & -1.71785500\end{array}$

$\begin{array}{llll}1.36909100 & -1.43534200 & -2.39907200\end{array}$

$\begin{array}{lll}4.17890900 & -2.04938400 & 1.77942400\end{array}$

$\begin{array}{llll}3.52032600 & -1.56209000 & 2.50732200\end{array}$

$\begin{array}{llll}4.77915100 & -2.79099900 & 2.31724900\end{array}$

$\begin{array}{llll}4.86089300 & -1.29725600 & 1.37326000\end{array}$

$\begin{array}{llll}2.45803600 & -3.83426400 & 1.27046700\end{array}$

$\begin{array}{llll}1.96167900 & -4.42413500 & 0.49615400\end{array}$

$\begin{array}{llll}3.05942500 & -4.52029200 & 1.87680600\end{array}$

$\begin{array}{lll}1.68922700 & -3.40057400 & 1.92009700\end{array}$

$\begin{array}{llll}0.11169200 & -2.77538600 & -1.29827500\end{array}$

$\begin{array}{llll}-0.48338600 & -1.96899000 & -0.86451300\end{array}$

$\begin{array}{llll}-0.41786900 & -3.14091700 & -2.18463300\end{array}$

$\begin{array}{lll}0.16148800 & -3.59660100 & -0.57769700\end{array}$

$\begin{array}{llll}2.30862900 & -3.37046000 & -2.43353200\end{array}$

$\begin{array}{llll}2.47926400 & -4.24038200 & -1.79161500\end{array}$

$\begin{array}{llll}1.74375400 & -3.71016900 & -3.30830200\end{array}$

$\begin{array}{llll}3.27497600 & -3.00499600 & -2.79108400\end{array}$

$\begin{array}{lll}-0.21425600 & -0.53666900 & 3.50760000\end{array}$

$\begin{array}{lll}-0.60232600 & 1.89517700 & 1.66964300\end{array}$

$\begin{array}{lll}0.86444300 & 1.98651400 & 3.34143700\end{array}$

$\begin{array}{lll}1.53165400 & 2.68563200 & 2.82768400\end{array}$

$\begin{array}{lll}1.49693500 & 1.25862400 & 3.86338200\end{array}$

$\begin{array}{llll}0.01155400 & 2.75585900 & 4.35645600\end{array}$

$\begin{array}{llll}-0.64330600 & 2.08079700 & 4.91772200\end{array}$

$\begin{array}{lll}0.65054800 & 3.28269000 & 5.07280000\end{array}$

$\begin{array}{llll}-0.61997400 & 3.49999900 & 3.85911900\end{array}$

$\begin{array}{llll}-2.83833100 & 0.05791900 & -0.23923000\end{array}$

$\begin{array}{lll}-4.09853800 & -0.65964900 & 0.46722800\end{array}$

$\begin{array}{llll}-2.92839500 & 0.91514100 & -1.60548600\end{array}$

$\begin{array}{lll}-3.74627700 & -0.86463600 & 1.87652600\end{array}$

$\begin{array}{lll}-5.42699900 & -0.14018900 & 0.14059500\end{array}$

$\begin{array}{lll}-4.06117500 & -2.37586400 & -0.25522200\end{array}$

$\begin{array}{llll}-1.58934900 & 0.94407000 & -2.19872700\end{array}$

$\begin{array}{llll}-4.07964300 & 0.65163600 & -2.47021400\end{array}$

$\begin{array}{llll}-3.18131200 & 2.65311700 & -0.98355200\end{array}$

$\begin{array}{lll}-2.93105100 & -2.99896000 & 0.09267900\end{array}$

$\begin{array}{llll}-5.09571700 & -3.07415600 & 0.21650000\end{array}$

$\begin{array}{lll}-4.13537600 & -2.33263400 & -1.58431300\end{array}$

$\begin{array}{llll}-2.14357800 & 3.03444300 & -0.23142600\end{array}$

$\begin{array}{llll}-3.28026700 & 3.48213800 & -2.02405100\end{array}$

$\begin{array}{llll}-4.29212500 & 2.73823800 & -0.25344300\end{array}$

Sum of electronic and zero-point Energies $=\quad-3082.684845$

Sum of electronic and thermal Free Energies $=\quad-3082.766713$

$\begin{array}{lrrr}\text { cis-3'b } \boldsymbol{\beta} & & & \\ \mathrm{Au} & 1.12919400 & 0.07577100 & 0.96121300 \\ \mathrm{C} & -0.00415500 & 1.23764700 & 2.27424500 \\ \mathrm{C} & -0.53773000 & -0.09924100 & 2.70971500 \\ \mathrm{H} & -0.07108400 & -0.99439000 & 2.12829200 \\ \mathrm{H} & -1.59736200 & -0.25243600 & 2.48994900 \\ \mathrm{P} & 2.42196200 & -1.54187600 & -0.26412800 \\ \mathrm{C} & 2.20135000 & 1.46809600 & -0.06630500 \\ \mathrm{C} & 3.29897400 & 1.00249500 & -0.85854500 \\ \mathrm{C} & 1.91691800 & 2.81628600 & -0.01437900 \\ \mathrm{C} & 3.61299200 & -0.38395400 & -1.00950600 \\ \mathrm{C} & 4.12351600 & 1.96464900 & -1.53038500 \\ \mathrm{C} & 2.71374200 & 3.75573100 & -0.71225900 \\ \mathrm{H} & 1.07655600 & 3.18249900 & 0.56456900 \\ \mathrm{C} & 4.72431800 & -0.77847600 & -1.73009500 \\ \mathrm{C} & 5.24775700 & 1.51505200 & -2.26948500 \\ \mathrm{C} & 3.80149300 & 3.34271700 & -1.44056800 \\ \mathrm{H} & 2.45928600 & 4.81046100 & -0.65289400 \\ \mathrm{C} & 5.55301400 & 0.17728300 & -2.35565500 \\ \mathrm{H} & 4.97230300 & -1.83150300 & -1.82225300\end{array}$




\begin{tabular}{|c|c|c|c|}
\hline $\mathrm{H}$ & 5.86995000 & 2.25509800 & -2.76669500 \\
\hline $\mathrm{H}$ & 4.42902100 & 4.06200900 & -1.96035500 \\
\hline $\mathrm{H}$ & 6.42287000 & -0.15266500 & -2.91597800 \\
\hline $\mathrm{C}$ & 3.38898100 & -2.76221100 & 0.75042900 \\
\hline $\mathrm{H}$ & 4.07318600 & -3.26121600 & 0.05156000 \\
\hline $\mathrm{C}$ & 1.55024700 & -2.39695900 & -1.67819100 \\
\hline $\mathrm{H}$ & 1.44077800 & -1.58119000 & -2.40459800 \\
\hline $\mathrm{C}$ & 4.21029900 & -2.02430600 & 1.81045500 \\
\hline $\mathrm{H}$ & 3.56207600 & -1.49703100 & 2.51989200 \\
\hline $\mathrm{H}$ & 4.80327700 & -2.74942500 & 2.37855000 \\
\hline $\mathrm{H}$ & 4.90063800 & -1.29830400 & 1.37204800 \\
\hline $\mathrm{C}$ & 2.47614400 & -3.81287500 & 1.38649000 \\
\hline $\mathrm{H}$ & 1.95513300 & -4.42057500 & 0.64250300 \\
\hline $\mathrm{H}$ & 3.07990100 & -4.48896600 & 2.00206900 \\
\hline $\mathrm{H}$ & 1.72616000 & -3.35295600 & 2.03996700 \\
\hline $\mathrm{C}$ & 0.14784100 & -2.85645300 & -1.27195200 \\
\hline $\mathrm{H}$ & -0.45247900 & -2.02666900 & -0.89156700 \\
\hline $\mathrm{H}$ & -0.36726500 & -3.26148000 & -2.14990500 \\
\hline $\mathrm{H}$ & 0.17427400 & -3.64312900 & -0.51193900 \\
\hline $\mathrm{C}$ & 2.36462500 & -3.52110700 & -2.32004000 \\
\hline $\mathrm{H}$ & 2.51854700 & -4.35630700 & -1.62942000 \\
\hline $\mathrm{H}$ & 1.81651700 & -3.90639800 & -3.18710600 \\
\hline $\mathrm{H}$ & 3.34043200 & -3.18114700 & -2.67678500 \\
\hline $\mathrm{H}$ & -0.28014300 & -0.36041500 & 3.73944800 \\
\hline $\mathrm{H}$ & -0.72058700 & 1.82529400 & 1.69786300 \\
\hline $\mathrm{C}$ & 0.75378000 & 2.05077400 & 3.30300700 \\
\hline $\mathrm{H}$ & 1.33854800 & 2.82808000 & 2.79856300 \\
\hline $\mathrm{H}$ & 1.46900600 & 1.40838600 & 3.83232000 \\
\hline $\mathrm{C}$ & -0.18825700 & 2.71273300 & 4.31496900 \\
\hline $\mathrm{H}$ & -0.77508700 & 1.96758600 & 4.86332100 \\
\hline $\mathrm{H}$ & 0.37860400 & 3.29797000 & 5.04701400 \\
\hline $\mathrm{H}$ & -0.89074300 & 3.38870500 & 3.81497800 \\
\hline $\mathrm{N}$ & -2.84392800 & 0.03782000 & -0.24367300 \\
\hline$S$ & -4.12277100 & -0.67461800 & 0.43462200 \\
\hline S & -2.89619400 & 0.87244200 & -1.62490200 \\
\hline $\mathrm{O}$ & -3.82699000 & -0.84188900 & 1.86144500 \\
\hline $\mathrm{O}$ & -5.44285600 & -0.17929300 & 0.04265600 \\
\hline $\mathrm{C}$ & -4.03466300 & -2.40535700 & -0.24654400 \\
\hline $\mathrm{O}$ & -1.53825500 & 0.90506000 & -2.17390400 \\
\hline $\mathrm{O}$ & -4.01516200 & 0.58457400 & -2.52355800 \\
\hline $\mathrm{C}$ & -3.18576100 & 2.61723800 & -1.03971000 \\
\hline $\mathrm{F}$ & -2.90124000 & -2.99761300 & 0.14276900 \\
\hline $\mathrm{F}$ & -5.06648200 & -3.11520500 & 0.21357100 \\
\hline $\mathrm{F}$ & -4.07570200 & -2.39139600 & -1.57793800 \\
\hline $\mathrm{F}$ & -2.17043500 & 3.02595900 & -0.27184800 \\
\hline $\mathrm{F}$ & -3.27323200 & 3.42704100 & -2.09634700 \\
\hline $\mathrm{F}$ & -4.31330200 & 2.69968000 & -0.33514200 \\
\hline
\end{tabular}

Sum of electronic and zero-point Energies $=\quad-3082.697908$ Sum of electronic and thermal Free Energies $=\quad-3082.779594$

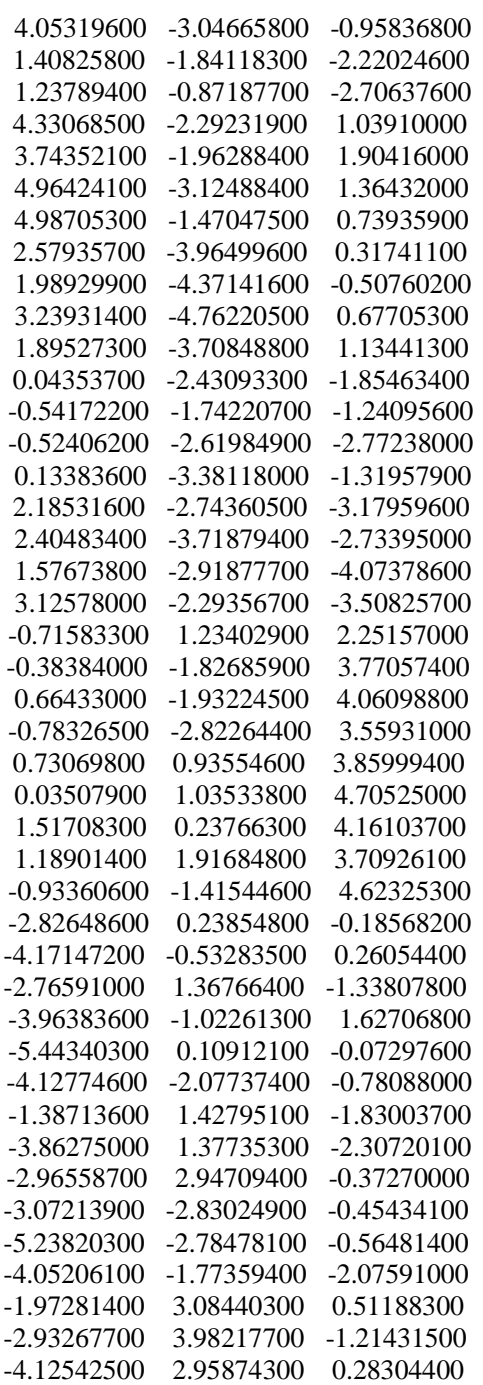

Sum of electronic and zero-point Energies=

Sum of electronic and thermal Free Energies=

$-3082.697256$ $-3082.777084$

\section{TS $^{1}\left(3^{\prime} \mathbf{b} \beta-3\right.$ 'bH)}

$\begin{array}{lccc}\mathrm{Au} & 1.12079000 & -0.25061300 & 0.97833100 \\ \mathrm{C} & 0.08802100 & 0.61800000 & 2.72570700 \\ \mathrm{C} & -0.56227900 & -0.63961700 & 2.61714000 \\ \mathrm{H} & 0.21857300 & -1.56935500 & 1.33191600 \\ \mathrm{H} & -1.48169300 & -0.64967300 & 2.03282300 \\ \mathrm{P} & 2.30615700 & -1.33930000 & -0.71128900 \\ \mathrm{C} & 2.27744700 & 1.40560600 & 0.45354800 \\ \mathrm{C} & 3.33663700 & 1.19667200 & -0.49178300 \\ \mathrm{C} & 2.09614900 & 2.68644400 & 0.94285200 \\ \mathrm{C} & 3.55737400 & -0.07719200 & -1.09951400 \\ \mathrm{C} & 4.20694700 & 2.27477300 & -0.85731400 \\ \mathrm{C} & 2.93266200 & 3.76328200 & 0.56015000 \\ \mathrm{H} & 1.29325000 & 2.89639700 & 1.64322700 \\ \mathrm{C} & 4.62009600 & -0.28730900 & -1.95795700 \\ \mathrm{C} & 5.28019400 & 2.02714200 & -1.75167100 \\ \mathrm{C} & 3.97603400 & 3.56240900 & -0.31006200 \\ \mathrm{H} & 2.74680800 & 4.75237500 & 0.97117500 \\ \mathrm{C} & 5.49391200 & 0.77424200 & -2.27792100 \\ \mathrm{H} & 4.79437600 & -1.26560600 & -2.39609200 \\ \mathrm{H} & 5.93959400 & 2.85160100 & -2.01222900 \\ \mathrm{H} & 4.63360500 & 4.38037600 & -0.59339400 \\ \mathrm{H} & 6.32688900 & 0.59629200 & -2.95178300 \\ \mathrm{C} & 3.19195000 & -2.86530400 & -0.14488200 \\ \mathrm{H} & 3.84810100 & -3.13571400 & -0.98319200 \\ \mathrm{C} & 1.31334100 & -1.63831400 & -2.25958500 \\ \mathrm{H} & 1.22899200 & -0.62310300 & -2.66815000 \\ \mathrm{C} & 4.05230300 & -2.54090000 & 1.07899200 \\ \mathrm{H} & 3.43005300 & -2.25887800 & 1.93597900\end{array}$




$\begin{array}{lrrr}\mathrm{H} & 4.62784400 & -3.42882100 & 1.36199100 \\ \mathrm{H} & 4.75978200 & -1.72938100 & 0.88691700 \\ \mathrm{C} & 2.23205800 & -4.02229600 & 0.13322000 \\ \mathrm{H} & 1.67633500 & -4.32721400 & -0.75665200 \\ \mathrm{H} & 2.80531500 & -4.88869500 & 0.48066200 \\ \mathrm{H} & 1.51156900 & -3.76240700 & 0.91691000 \\ \mathrm{C} & -0.09698200 & -2.15033700 & -1.96181700 \\ \mathrm{H} & -0.63869500 & -1.47995100 & -1.29136100 \\ \mathrm{H} & -0.65743000 & -2.20555700 & -2.90132000 \\ \mathrm{H} & -0.08816400 & -3.15210500 & -1.52269700 \\ \mathrm{C} & 2.04498000 & -2.51930900 & -3.27510700 \\ \mathrm{H} & 2.16289100 & -3.54509300 & -2.91281400 \\ \mathrm{H} & 1.45276600 & -2.56035300 & -4.19578800 \\ \mathrm{H} & 3.03038000 & -2.12642600 & -3.53841700 \\ \mathrm{H} & -0.45074200 & 1.45403600 & 2.28169900 \\ \mathrm{C} & -0.41833500 & -1.74133500 & 3.62748800 \\ \mathrm{H} & 0.60365700 & -1.85706100 & 3.99283200 \\ \mathrm{H} & -0.76830900 & -2.69747800 & 3.23235300 \\ \mathrm{C} & 1.00538700 & 0.95172500 & 3.87312800 \\ \mathrm{H} & 0.40636300 & 1.18299800 & 4.76419400 \\ \mathrm{H} & 1.67769600 & 0.12907600 & 4.13080900 \\ \mathrm{H} & 1.61314800 & 1.83154000 & 3.65114000 \\ \mathrm{H} & -1.05319700 & -1.48180500 & 4.48514600 \\ \mathrm{~N} & -2.80777100 & 0.26908100 & -0.18693800 \\ \mathrm{~S} & -4.13614500 & -0.47666900 & 0.34257200 \\ \mathrm{~S} & -2.79278200 & 1.37660600 & -1.36220000 \\ \mathrm{O} & -3.85328600 & -0.96038400 & 1.69791800 \\ \mathrm{O} & -5.41500800 & 0.18459800 & 0.08186200 \\ \mathrm{C} & -4.17589300 & -2.02899100 & -0.68580100 \\ \mathrm{O} & -1.44320100 & 1.40395700 & -1.93120400 \\ \mathrm{O} & -3.94284800 & 1.38707600 & -2.26749100 \\ \mathrm{C} & -2.91019700 & 2.97431400 & -0.41340800 \\ \mathrm{~F} & -3.07829800 & -2.75931900 & -0.46361400 \\ \mathrm{~F} & -5.24470200 & -2.75435700 & -0.35110200 \\ \mathrm{~F} & -4.24019400 & -1.73566600 & -1.98360200 \\ \mathrm{~F} & -1.86349200 & 3.10634000 & 0.40796200 \\ \mathrm{~F} & -2.90961400 & 3.99591600 & -1.27170500 \\ & -4.02852100 & 3.01499000 & 0.30948500\end{array}$

Sum of electronic and zero-point Energies $=\quad-3082.688444$ Sum of electronic and thermal Free Energies $=-3082.768252$

$\begin{array}{cccc}\text { trans }{ }^{1} \mathbf{- 3} \mathbf{b H}^{\prime} & & & \\ \mathrm{Au} & 1.11534000 & -0.21332400 & 0.92148300 \\ \mathrm{C} & 0.05379300 & 0.99617600 & 2.57068100 \\ \mathrm{C} & -0.61564600 & -0.22104100 & 2.59929800 \\ \mathrm{H} & 0.44817300 & -1.65047700 & 1.21609300 \\ \mathrm{H} & -1.47492400 & -0.32350900 & 1.93823500 \\ \mathrm{P} & 2.46145500 & -1.32570300 & -0.58295700 \\ \mathrm{C} & 2.19228100 & 1.49992100 & 0.28790200 \\ \mathrm{C} & 3.35652000 & 1.26012700 & -0.51851400 \\ \mathrm{C} & 1.89130500 & 2.81849200 & 0.57972400 \\ \mathrm{C} & 3.70168300 & -0.05346800 & -0.95410500 \\ \mathrm{C} & 4.20938700 & 2.33956200 & -0.92081300 \\ \mathrm{C} & 2.70603200 & 3.89574500 & 0.15260000 \\ \mathrm{H} & 1.00820600 & 3.06738900 & 1.15975200 \\ \mathrm{C} & 4.85839400 & -0.30007900 & -1.66897600 \\ \mathrm{C} & 5.38581800 & 2.05610100 & -1.66189100 \\ \mathrm{C} & 3.85341500 & 3.66472200 & -0.56532600 \\ \mathrm{H} & 2.41983600 & 4.91179700 & 0.41242400 \\ \mathrm{C} & 5.71466700 & 0.76741800 & -2.01412700 \\ \mathrm{H} & 5.11533700 & -1.30948100 & -1.97597300 \\ \mathrm{H} & 6.03074200 & 2.88352400 & -1.94803300 \\ \mathrm{H} & 4.49542700 & 4.48541600 & -0.87514100 \\ \mathrm{H} & 6.62383600 & 0.56483400 & -2.57266900 \\ \mathrm{C} & 3.34142300 & -2.78445700 & 0.13775300 \\ \mathrm{H} & 4.09869100 & -3.03136600 & -0.61926200 \\ \mathrm{C} & 1.57486200 & -1.72811300 & -2.16914100 \\ \mathrm{H} & 1.45181100 & -0.73082000 & -2.61129400 \\ \mathrm{C} & 4.04729200 & -2.37342200 & 1.43251900 \\ \mathrm{H} & 3.32042800 & -2.12357200 & 2.21339500 \\ \mathrm{H} & 4.65190600 & -3.21205700 & 1.79361800 \\ \mathrm{H} & 4.71181300 & -1.51632600 & 1.29106000 \\ \mathrm{C} & 2.43012900 & -3.99251200 & 0.34837500 \\ \mathrm{H} & 2.01143700 & -4.36881400 & -0.58800700 \\ \mathrm{H} & 3.01292700 & -4.80167300 & 0.80156900\end{array}$

$\begin{array}{lll}1.60311900 & -3.75523800 & 1.02659200\end{array}$ $\begin{array}{llll}0.18422300 & -2.32358300 & -1.94199100\end{array}$ $-0.43711100-1.67560400-1.32022300$ $-0.31181200 \quad-2.43180700 \quad-2.91254100$ $\begin{array}{llll}0.22792500 & -3.31311000 & -1.47895900\end{array}$ $\begin{array}{llll}2.42838100 & -2.58279900 & -3.11007000\end{array}$ $2.60272800-3.58414800 \quad-2.70510600$ $\begin{array}{llll}1.89207700 & -2.69729300 & -4.05821000\end{array}$ $\begin{array}{llll}3.39408700 & -2.12289700 & -3.33525800\end{array}$ $\begin{array}{lll}-0.39743100 & 1.76658600 & 1.94886800\end{array}$ $\begin{array}{lll}-0.48725500 & -1.24462900 & 3.68455000\end{array}$ $\begin{array}{llll}0.51947100 & -1.30928800 & 4.10221400\end{array}$ $\begin{array}{lll}-0.79715400 & -2.23391900 & 3.34188400\end{array}$ $\begin{array}{lll}0.99855300 & 1.45758600 & 3.64480900\end{array}$ $\begin{array}{llll}0.41507100 & 1.89546000 & 4.46557300\end{array}$ $\begin{array}{lll}1.59608000 & 0.64313200 & 4.06207300\end{array}$ $\begin{array}{lll}1.67639000 & 2.23015300 & 3.27649000\end{array}$ $\begin{array}{lll}-1.16464300 & -0.94954900 & 4.49778200\end{array}$ $\begin{array}{llll}-2.87596000 & 0.19515000 & -0.15377600\end{array}$ $\begin{array}{lll}-4.14394100 & -0.66820900 & 0.34819600\end{array}$ $\begin{array}{llll}-2.92764100 & 1.26982500 & -1.35761600\end{array}$

$\begin{array}{lll}-3.86466800 & -1.09317600 & 1.72300000\end{array}$

$\begin{array}{llll}-5.46990200 & -0.13874500 & 0.02686100\end{array}$ $\begin{array}{llll}-3.99733900 & -2.23899500 & -0.64185200\end{array}$ $\begin{array}{llll}-1.55847500 & 1.44891500 & -1.84768400\end{array}$ $\begin{array}{llll}-4.01262800 & 1.11479200 & -2.32776800\end{array}$ $\begin{array}{llll}-3.29880000 & 2.86666200 & -0.47276700\end{array}$ $\begin{array}{llll}-2.84499800 & -2.85684000 & -0.36315700\end{array}$ $\begin{array}{lll}-5.00538800 & -3.05395500 & -0.32473700\end{array}$ $\begin{array}{llll}-4.04088400 & -1.98282600 & -1.94827500\end{array}$ $\begin{array}{llll}-2.35068700 & 3.13197500 & 0.43140900\end{array}$ $\begin{array}{llll}-3.33056200 & 3.86199900 & -1.36107500\end{array}$ $\begin{array}{lll}-4.47530800 & 2.80055100 & 0.14808700\end{array}$

Sum of electronic and zero-point Energies= $-3082.689221$ Sum of electronic and thermal Free Energies $=\quad-3082.767202$

$\begin{array}{lrrr}\text { cis-3''b } \boldsymbol{\beta} & & & \\ \mathrm{Au} & 1.56868000 & 0.16136800 & 1.15975800 \\ \mathrm{C} & 0.85817000 & 1.44623100 & 2.64736400 \\ \mathrm{C} & 0.31797700 & 0.17592400 & 3.25107800 \\ \mathrm{H} & 0.63071900 & -0.75611400 & 2.60635200 \\ \mathrm{P} & 2.38823300 & -1.59157700 & -0.27039100 \\ \mathrm{C} & 2.42518200 & 1.43547800 & -0.17478700 \\ \mathrm{C} & 3.21917900 & 0.86319800 & -1.21906700 \\ \mathrm{C} & 2.26515100 & 2.80337700 & -0.10512200 \\ \mathrm{C} & 3.37915400 & -0.54699900 & -1.38679600 \\ \mathrm{C} & 3.88636300 & 1.73946600 & -2.13734000 \\ \mathrm{C} & 2.89883600 & 3.65855500 & -1.03845900 \\ \mathrm{H} & 1.64862900 & 3.24912100 & 0.66749400 \\ \mathrm{C} & 4.21203700 & -1.04727500 & -2.37007300 \\ \mathrm{C} & 4.72005900 & 1.18291800 & -3.14108100 \\ \mathrm{C} & 3.70270900 & 3.14081500 & -2.02333900 \\ \mathrm{H} & 2.74808000 & 4.73159900 & -0.95825100 \\ \mathrm{C} & 4.89320200 & -0.17675900 & -3.24760600 \\ \mathrm{H} & 4.35243300 & -2.11889400 & -2.47762900 \\ \mathrm{H} & 5.22644500 & 1.85833100 & -3.82621100 \\ \mathrm{H} & 4.20651800 & 3.79331900 & -2.73165900 \\ \mathrm{H} & 5.54352000 & -0.58936700 & -4.01333700 \\ \mathrm{C} & 3.56234200 & -2.82332600 & 0.47703300 \\ \mathrm{H} & 4.01817700 & -3.34332300 & -0.37589700 \\ \mathrm{C} & 1.09689200 & -2.43657600 & -1.32039300 \\ \mathrm{H} & 0.79337000 & -1.62627800 & -1.99582200 \\ \mathrm{C} & 4.65744200 & -2.08754200 & 1.25284300 \\ \mathrm{H} & 4.24094000 & -1.54912300 & 2.11184300 \\ \mathrm{H} & 5.38199600 & -2.81479400 & 1.63537400 \\ \mathrm{H} & 5.19955600 & -1.37085100 & 0.62949200 \\ \mathrm{C} & 2.84419400 & -3.84502400 & 1.36045800 \\ \mathrm{H} & 2.12161000 & -4.44577900 & 0.80286300 \\ \mathrm{H} & 3.58260400 & -4.53057000 & 1.79092800 \\ \mathrm{H} & 2.31895000 & -3.36199200 & 2.19214100 \\ \mathrm{C} & -0.12622000 & -2.84105000 & -0.49266300 \\ \mathrm{H} & -0.54982200 & -1.99065300 & 0.04851500 \\ \mathrm{H} & -0.90220400 & -3.22130200 & -1.16500000 \\ \mathrm{H} & 0.10466200 & -3.63231800 & 0.22697800\end{array}$




$\begin{array}{lrrr}\mathrm{C} & 1.63369300 & -3.59876600 & -2.15640100 \\ \mathrm{H} & 1.97853300 & -4.42828200 & -1.53108100 \\ \mathrm{H} & 0.82756600 & -3.97928500 & -2.79358400 \\ \mathrm{H} & 2.45261000 & -3.29549000 & -2.81382100 \\ \mathrm{H} & 0.08152600 & 2.09064900 & 2.23099700 \\ \mathrm{C} & 1.90521500 & 2.19667000 & 3.43593800 \\ \mathrm{H} & 1.44076600 & 2.68088500 & 4.30699100 \\ \mathrm{H} & 2.69233800 & 1.53339900 & 3.80992800 \\ \mathrm{H} & 2.37444900 & 2.98504800 & 2.84062400 \\ \mathrm{H} & 0.83404800 & -0.07202900 & 4.18492200 \\ \mathrm{C} & -1.19786800 & 0.06565000 & 3.35700000 \\ \mathrm{H} & -1.68080400 & 0.27066900 & 2.39843700 \\ \mathrm{H} & -1.50331700 & -0.92993400 & 3.69083600 \\ \mathrm{H} & -1.56104100 & 0.79761500 & 4.08578800 \\ \mathrm{~N} & -3.19336200 & 0.04174600 & -0.07649800 \\ \mathrm{~S} & -4.64923500 & -0.55232500 & 0.29353100 \\ \mathrm{~S} & -2.91653700 & 1.08211900 & -1.27728800 \\ \mathrm{O} & -4.61054900 & -0.98577900 & 1.69143400 \\ \mathrm{O} & -5.80714200 & 0.20854500 & -0.17967000 \\ \mathrm{C} & -4.69171500 & -2.14214500 & -0.67482900 \\ \mathrm{O} & -1.50469900 & 0.96267200 & -1.65323600 \\ \mathrm{O} & -3.92375400 & 1.15223400 & -2.33725800 \\ \mathrm{C} & -3.01126200 & 2.73220400 & -0.42005100 \\ \mathrm{~F} & -3.73178200 & -2.96936300 & -0.25388800 \\ \mathrm{~F} & -5.87452600 & -2.73591400 & -0.49862800 \\ \mathrm{~F} & -4.51643500 & -1.90793600 & -1.97548600 \\ \mathrm{~F} & -2.07294300 & 2.82053800 & 0.52888000 \\ \mathrm{~F} & -2.80785600 & 3.70548600 & -1.31030400 \\ \mathrm{~F} & -4.20550400 & 2.90395400 & 0.14497400\end{array}$

Sum of electronic and zero-point Energies $=\quad-3082.696568$ Sum of electronic and thermal Free Energies $=\quad-3082.777032$

\begin{tabular}{|c|c|c|c|}
\hline \multicolumn{4}{|c|}{$\operatorname{TS}\left(3^{\prime} b_{\beta}-3^{\prime} b_{H}\right)$} \\
\hline $\mathrm{Au}$ & 1.63720800 & 0.01404900 & 1.18450700 \\
\hline $\mathrm{C}$ & 0.99755700 & 1.36257700 & 2.80329300 \\
\hline $\mathrm{C}$ & 0.51735400 & 0.11459100 & 3.27613100 \\
\hline $\mathrm{H}$ & 1.14280000 & -1.15494600 & 2.22378600 \\
\hline $\mathrm{P}$ & 2.43272800 & -1.52517500 & -0.37869700 \\
\hline $\mathrm{C}$ & 2.32995900 & 1.44831900 & -0.16337800 \\
\hline $\mathrm{C}$ & 3.10581700 & 0.98027200 & -1.27615400 \\
\hline $\mathrm{C}$ & 2.12114000 & 2.81085400 & -0.05146300 \\
\hline $\mathrm{C}$ & 3.32736000 & -0.41231500 & -1.50251000 \\
\hline $\mathrm{C}$ & 3.68887300 & 1.91005500 & -2.19742900 \\
\hline $\mathrm{C}$ & 2.66585400 & 3.73208300 & -0.97921200 \\
\hline $\mathrm{H}$ & 1.52607300 & 3.20797800 & 0.76569100 \\
\hline $\mathrm{C}$ & 4.12887100 & -0.85392400 & -2.53824200 \\
\hline $\mathrm{C}$ & 4.49797700 & 1.42252100 & -3.25628300 \\
\hline $\mathrm{C}$ & 3.44503900 & 3.29595500 & -2.02280900 \\
\hline $\mathrm{H}$ & 2.46886500 & 4.79347700 & -0.85133100 \\
\hline $\mathrm{C}$ & 4.72591700 & 0.07536700 & -3.41719500 \\
\hline $\mathrm{H}$ & 4.30797500 & -1.91525200 & -2.68645800 \\
\hline $\mathrm{H}$ & 4.94171900 & 2.13958900 & -3.94279900 \\
\hline $\mathrm{H}$ & 3.88050500 & 3.99995700 & -2.72743000 \\
\hline $\mathrm{H}$ & 5.35566500 & -0.28226100 & -4.22661100 \\
\hline $\mathrm{C}$ & 3.64899200 & -2.75108600 & 0.29512500 \\
\hline $\mathrm{H}$ & 4.07410800 & -3.23862500 & -0.59283400 \\
\hline $\mathrm{C}$ & 1.07603400 & -2.33762500 & -1.36374400 \\
\hline $\mathrm{H}$ & 0.73561200 & -1.50644300 & -1.99441500 \\
\hline $\mathrm{C}$ & 4.76503800 & -2.01809100 & 1.04279100 \\
\hline $\mathrm{H}$ & 4.37435400 & -1.50400500 & 1.92820900 \\
\hline $\mathrm{H}$ & 5.50971600 & -2.74661300 & 1.38097100 \\
\hline $\mathrm{H}$ & 5.27558300 & -1.28286400 & 0.41468000 \\
\hline $\mathrm{C}$ & 2.98252200 & -3.80704400 & 1.17647800 \\
\hline $\mathrm{H}$ & 8200 & -4.40227700 & 0.63148600 \\
\hline $\mathrm{H}$ & 3.74866600 & -4.49154500 & 1.55669100 \\
\hline $\mathrm{H}$ & 2.48511000 & -3.35032400 & 2.03952500 \\
\hline $\mathrm{C}$ & -0.10150300 & -2.78216100 & -0.49375900 \\
\hline $\mathrm{H}$ & -0.51458100 & -1.95098000 & 0.08354000 \\
\hline $\mathrm{H}$ & -0.89756200 & -3.15798700 & -1.14465100 \\
\hline $\mathrm{H}$ & 0.17024900 & -3.58616200 & 0.19646300 \\
\hline $\mathrm{C}$ & 1.58719100 & -3.46181200 & -2.26760400 \\
\hline $\mathrm{H}$ & 1.95972900 & -4.31378100 & -1.69016500 \\
\hline $\mathrm{H}$ & 0.75680600 & -3.81865900 & -2.88648300 \\
\hline $\mathrm{H}$ & 2.37711700 & -3.12504100 & -2.94417800 \\
\hline $\mathrm{H}$ & 0.25397600 & 2.01693100 & 2.34822700 \\
\hline
\end{tabular}

$\begin{array}{rrr}2.17509000 & 2.03370800 & 3.46518400 \\ 1.82660700 & 2.60094300 & 4.33827000 \\ 2.91250500 & 1.30425500 & 3.81414900 \\ 2.67369300 & 2.73775400 & 2.79489900 \\ 1.11131800 & -0.37246900 & 4.05000000 \\ -0.93156600 & -0.26828700 & 3.21644500 \\ -1.43577900 & 0.15292600 & 2.34494500 \\ -1.07651000 & -1.35053700 & 3.22760400 \\ -1.41128900 & 0.14043200 & 4.11555900 \\ -3.24305800 & 0.02583500 & -0.03787900 \\ -4.70252000 & -0.57260900 & 0.30675400 \\ -2.96058400 & 1.15357000 & -1.15466100 \\ -4.64888300 & -1.12116300 & 1.66326700 \\ -5.85479400 & 0.24274500 & -0.08184500 \\ -4.78518600 & -2.07592700 & -0.78761600 \\ -1.56027000 & 1.02957000 & -1.56932900 \\ -3.98543000 & 1.33995000 & -2.18312300 \\ -2.99386800 & 2.72464700 & -0.15659500 \\ -3.80924400 & -2.93152700 & -0.47333700 \\ -5.96019300 & -2.68652200 & -0.61610000 \\ -4.66019600 & -1.73411300 & -2.07012000 \\ -2.04569000 & 2.69741800 & 0.78709200 \\ -2.76401100 & 3.76595400 & -0.95873500 \\ -4.17733400 & 2.88469600 & 0.43434000\end{array}$

Sum of electronic and zero-point Energies=

Sum of electronic and thermal Free Energies=

$-3082.687254$

$-3082.766733$ 


$\begin{array}{lrrr}\text { C } & -0.81917500 & -0.05277200 & 3.25474000 \\ \text { H } & -1.29743800 & 0.49443100 & 2.44026600 \\ \text { H } & -1.02486500 & -1.11915300 & 3.13961700 \\ \text { H } & -1.28451300 & 0.26688500 & 4.19733400 \\ \text { N } & -3.23940300 & 0.04244200 & -0.01095300 \\ \text { S } & -4.69347200 & -0.54247200 & 0.37430600 \\ \text { S } & -2.97191900 & 1.15348100 & -1.14730300 \\ \text { O } & -4.61537700 & -1.06678200 & 1.73914100 \\ \text { O } & -5.85090200 & 0.26935700 & -0.00630800 \\ \text { C } & -4.80105000 & -2.06417400 & -0.69181500 \\ \text { O } & -1.58697700 & 1.00318800 & -1.60260300 \\ \text { O } & -4.02379300 & 1.34817000 & -2.14675800 \\ \text { C } & -2.95158500 & 2.73397800 & -0.16414300 \\ \text { F } & -3.82794300 & -2.92166600 & -0.37418500 \\ \text { F } & -5.97838400 & -2.66294400 & -0.49676900 \\ \text { F } & -4.68805400 & -1.74543800 & -1.98165300 \\ \text { F } & -1.98200800 & 2.70041200 & 0.75742900 \\ \text { F } & -2.72302200 & 3.76322500 & -0.98220500 \\ \text { F } & -4.11821100 & 2.92063800 & 0.45240200\end{array}$

Sum of electronic and zero-point Energies $=\quad-3082.689031$ Sum of electronic and thermal Free Energies $=\quad-3082.768094$

\section{Olefin Rotation}

$\begin{array}{lccc}\text { TSrot1NTf2 } & & & \\ \mathrm{Au} & 1.40611700 & -0.23484600 & 1.12665300 \\ \mathrm{C} & 0.82988300 & 0.82992800 & 3.08909200 \\ \mathrm{C} & -0.37802000 & 0.23988700 & 2.80520300 \\ \mathrm{H} & 0.85556100 & -1.68261800 & 1.57039400 \\ \mathrm{H} & -1.07812300 & 0.78403800 & 2.17050300 \\ \mathrm{P} & 2.53846300 & -1.32012200 & -0.54881600 \\ \mathrm{C} & 2.31563300 & 1.49971500 & 0.31538400 \\ \mathrm{C} & 3.37458300 & 1.28218700 & -0.62962700 \\ \mathrm{C} & 2.00456900 & 2.81281300 & 0.61964200 \\ \mathrm{C} & 3.69374600 & -0.02854600 & -1.09144600 \\ \mathrm{C} & 4.13948500 & 2.37646300 & -1.14728300 \\ \mathrm{C} & 2.72664200 & 3.90579900 & 0.07976900 \\ \mathrm{H} & 1.17970700 & 3.04764300 & 1.28747300 \\ \mathrm{C} & 4.75031000 & -0.25819900 & -1.95209800 \\ \mathrm{C} & 5.22085500 & 2.11073200 & -2.02707800 \\ \mathrm{C} & 3.78826300 & 3.69625700 & -0.76627700 \\ \mathrm{H} & 2.44000600 & 4.91781800 & 0.35436700 \\ \mathrm{C} & 5.53157500 & 0.82554600 & -2.40855000 \\ \mathrm{H} & 4.98907800 & -1.26462800 & -2.28381200 \\ \mathrm{H} & 5.80617300 & 2.94851700 & -2.39859000 \\ \mathrm{H} & 4.36212600 & 4.53055500 & -1.16163300 \\ \mathrm{H} & 6.36752800 & 0.63765800 & -3.07588200 \\ \mathrm{C} & 3.52497400 & -2.76573600 & 0.04906500 \\ \mathrm{H} & 4.15760800 & -3.01981200 & -0.81336300 \\ \mathrm{C} & 1.44414900 & -1.73093500 & -1.99487100 \\ \mathrm{H} & 1.26841900 & -0.73640000 & -2.42516600 \\ \mathrm{H} & -0.92415000 & -0.97314200 & 3.49210900 \\ \mathrm{C} & -1.41566300 & -1.62509400 & 2.76452900 \\ \mathrm{H} & 3.82162100 & -2.05234900 & 2.08712300 \\ \mathrm{H} & 5.05537900 & -3.18119600 & 1.50576400 \\ \mathrm{H} & 5.07347700 & -1.50012200 & 0.95467100 \\ \mathrm{C} & 2.66435100 & -3.97545800 & 0.40993800 \\ \mathrm{H} & 2.08237800 & -4.34318100 & -0.43829900 \\ \mathrm{H} & 3.31955100 & -4.78746900 & 0.74316200 \\ \mathrm{H} & 1.97545400 & -3.74206200 & 1.22865600 \\ \mathrm{C} & 0.09277000 & -2.31978800 & -1.58732700 \\ \mathrm{H} & -0.44742000 & -1.65935200 & -0.90549900 \\ \mathrm{H} & -0.51839000 & -2.44146400 & -2.48764100 \\ \mathrm{H} & 0.19167500 & -3.30110800 & -1.11532900 \\ \mathrm{C} & 2.17471800 & -2.59276200 & -3.02914200 \\ \mathrm{H} & 2.38448300 & -3.59642000 & -2.64703000 \\ \mathrm{H} & 3.53197200 & -2.69949500 & -3.90932800 \\ \mathrm{H} & 1.01526700 & 1.86654200 & 2.83607800 \\ \mathrm{H} & & -0.53119200 & 4.54199900\end{array}$

$\begin{array}{lll}-1.49865300 & 0.10509100 & 5.30720400\end{array}$

$\begin{array}{lll}-2.78066000 & 0.02128300 & 4.08253900\end{array}$

$\begin{array}{lll}-2.37675300 & -1.41128600 & 5.03723000\end{array}$

$\begin{array}{lll}1.50048500 & 0.37479400 & 3.81727300\end{array}$

$\begin{array}{lll}-3.00087900 & 0.15625100 & -0.23322700\end{array}$

$\begin{array}{llll}-2.79879700 & 1.38544100 & -1.25801500\end{array}$

$\begin{array}{lll}-4.38780100 & -0.64659300 & -0.03473500\end{array}$

$\begin{array}{lll}-1.37292400 & 1.46307400 & -1.58820800\end{array}$

$\begin{array}{lll}-3.77893900 & 1.51729600 & -2.33711000\end{array}$

$\begin{array}{lll}-3.08520700 & 2.86667800 & -0.16727100\end{array}$

$-4.35348500-1.27236800 \quad 1.28887400$

$\begin{array}{lll}-5.61000400 & 0.03670000 & -0.46181800\end{array}$

$\begin{array}{lll}-4.20147500 & -2.07293500 & -1.21683600\end{array}$

$\begin{array}{lll}-2.18798300 & 2.89818300 & 0.82475100\end{array}$

$\begin{array}{lll}-2.95709900 & 3.98091500 & -0.89014700\end{array}$

$\begin{array}{lll}-4.30663600 & 2.83309400 & 0.36405200\end{array}$

$-3.17191300-2.84587400 \quad-0.86054000$

$-5.31432200-2.80994700-1.19689300$

$-3.99770700 \quad-1.63286600 \quad-2.45888400$

Sum of electronic and zero-point Energies $=\quad-3082.681263$ Sum of electronic and thermal Free Energies $=\quad-3082.762552$

TSrot1NTf2-complex between both TS rotation

$\mathrm{Au} \quad 1.07970000 \quad-0.39187000 \quad 0.81304700$

$\begin{array}{llll}\text { C } & 0.56332300 & -0.05531900 & 3.16708200\end{array}$

$\begin{array}{llll}\text { C } & -0.49984100 & 0.29381200 & 2.37279000\end{array}$

$\mathrm{H} \quad 0.32817900-1.82102100 \quad 0.83334400$

$\begin{array}{llll}\mathrm{H} & -0.64094300 & 1.32283400 & 2.05331300\end{array}$

$\begin{array}{llll}\mathrm{P} & 2.30258700 & -1.07314700 & -1.01436600\end{array}$

$\begin{array}{llll}2.20781500 & 1.38323100 & 0.63463100\end{array}$

$\begin{array}{llll}3.30807600 & 1.36831500 & -0.28505100\end{array}$

$\begin{array}{lll}1.99985800 & 2.54088700 & 1.36171700\end{array}$

$\begin{array}{lll}3.56105000 & 0.23500800 & -1.11199600\end{array}$

$\begin{array}{llll}4.19050900 & 2.49060400 & -0.39392900\end{array}$

$\begin{array}{llll}2.84779300 & 3.66949900 & 1.23702700\end{array}$

$\begin{array}{lll}1.16910300 & 2.61319300 & 2.06020000\end{array}$

$\begin{array}{llll}4.65429700 & 0.18684400 & -1.95619400\end{array}$

$\begin{array}{llll}5.29853200 & 2.41576400 & -1.27706400\end{array}$

$\begin{array}{llll}3.92730300 & 3.64464800 & 0.38735000\end{array}$

$\begin{array}{llll}2.64083500 & 4.55735100 & 1.82919600\end{array}$

$\begin{array}{llll}5.53542700 & 1.28767600 & -2.02890600\end{array}$

$\begin{array}{llll}4.84856600 & -0.69002700 & -2.56662500\end{array}$

$\begin{array}{llll}5.96828000 & 3.26968800 & -1.34547500\end{array}$

$\begin{array}{llll}4.59042700 & 4.50177400 & 0.30206900\end{array}$

$\begin{array}{llll}6.39404800 & 1.24073100 & -2.69234400\end{array}$

$\begin{array}{llll}3.17485400 & -2.68875800 & -0.78800700\end{array}$

$\begin{array}{llll}3.84277100 & -2.73953400 & -1.65982900\end{array}$

$\begin{array}{llll}1.30409200 & -1.01284300 & -2.58251300\end{array}$

$\begin{array}{llll}1.20653600 & 0.06880100 & -2.74191800\end{array}$

$\begin{array}{llll}4.02280400 & -2.65421400 & 0.48538000\end{array}$

$\begin{array}{llll}3.39099600 & -2.58781300 & 1.37772400\end{array}$

$\begin{array}{lll}4.60208000 & -3.58102800 & 0.55460500\end{array}$

$\begin{array}{llll}4.72681700 & -1.81722000 & 0.49529600\end{array}$

$\begin{array}{llll}2.23101700 & -3.89041800 & -0.81063000\end{array}$

$\begin{array}{llll}1.68494700 & -3.97680800 & -1.75288500\end{array}$

$\begin{array}{llll}2.82033600 & -4.80462600 & -0.68192900\end{array}$

$\begin{array}{llll}1.50388500 & -3.84186000 & 0.00653900\end{array}$

$\begin{array}{lll}-0.09941300 & -1.59970500 & -2.42442300\end{array}$

$\begin{array}{llll}-0.65291100 & -1.10420200 & -1.62504000\end{array}$

$\begin{array}{llll}-0.64822400 & -1.44364700 & -3.35919000\end{array}$

$-0.07901800 \quad-2.67402200 \quad-2.22382800$

$\begin{array}{llll}2.06256800 & -1.61800400 & -3.76724200\end{array}$

$\begin{array}{lll}2.20492500 & -2.69703700 & -3.65335000\end{array}$

$\begin{array}{llll}1.47215000 & -1.45556900 & -4.67527700\end{array}$

$\begin{array}{lll}3.03791200 & -1.14960700 & -3.92421200\end{array}$

$\begin{array}{llll}1.29985500 & 0.70903100 & 3.41563900\end{array}$

$\begin{array}{llll}-2.82568700 & 0.30963000 & -0.15541100\end{array}$

$\begin{array}{lll}-2.99200200 & 1.42343500 & -1.30911100\end{array}$

$-4.00462100 \quad-0.65545200 \quad 0.36816200$

$\begin{array}{llll}-1.66075000 & 1.70232700 & -1.85402200\end{array}$

$\begin{array}{llll}-4.11148100 & 1.25097200 & -2.23659600\end{array}$

$\begin{array}{llll}-3.40499500 & 2.95145200 & -0.32751800\end{array}$

$\begin{array}{lll}-3.69287800 & -1.03361100 & 1.75030900\end{array}$

$\begin{array}{llll}-5.37602100 & -0.26617900 & 0.03703700\end{array}$

$\begin{array}{llll}-3.70254100 & -2.21900900 & -0.59876600\end{array}$ 


$\begin{array}{ccc}-2.40126300 & 3.25761200 & 0.49961600 \\ -3.60054800 & 3.97581300 & -1.15993700 \\ -4.51113100 & 2.76193600 & 0.39220100 \\ -2.54758800 & -2.78023100 & -0.22701000 \\ -4.69121600 & -3.08262000 & -0.36041100 \\ -3.65340300 & -1.96561600 & -1.90689600 \\ -1.34014100 & -0.38054600 & 2.22920300 \\ 0.69339800 & -1.35373900 & 3.89865700 \\ -0.02143500 & -2.08196900 & 3.50322300 \\ 1.70248800 & -1.75717200 & 3.75293900 \\ 0.45929000 & -1.13427500 & 5.40069100 \\ -0.55072900 & -0.75856600 & 5.59195400 \\ 0.57748400 & -2.08132200 & 5.93592600 \\ 1.17552200 & -0.41887600 & 5.81814300\end{array}$

Sum of electronic and zero-point Energies $=\quad-3082.684923$ Sum of electronic and thermal Free Energies $=\quad-3082.765564$

\section{TSrot2NTf2}

$\begin{array}{llll}\mathrm{Au} & 1.12514600 & -0.42846100 & 0.86027700\end{array}$

$\begin{array}{llll}0.51915100 & 0.29424800 & 3.07529900\end{array}$

$\begin{array}{llll}\mathrm{C} & -0.54941900 & -0.34526100 & 2.49689200\end{array}$

$\mathrm{H} \quad 0.65997100 \quad-1.96449400 \quad 1.02381400$

$\mathrm{H} \quad-1.30817700 \quad 0.20411300 \quad 1.94286400$

$\begin{array}{llll}\mathrm{P} & 2.41338200 & -1.16651200 & -0.89370800\end{array}$

$\begin{array}{llll}\mathrm{C} & 1.94241800 & 1.46774900 & 0.38784200\end{array}$

$\begin{array}{llll}\text { C } & 3.04914600 & 1.47173200 & -0.52655100\end{array}$

$\begin{array}{llll}\mathrm{C} & 1.52137400 & 2.69179700 & 0.87525700\end{array}$

$\begin{array}{llll}\text { C } & 3.48399900 & 0.27519000 & -1.17033100\end{array}$

$\begin{array}{llll}\text { C } & 3.74558000 & 2.68522300 & -0.83381700\end{array}$

$\begin{array}{llll}\mathrm{C} & 2.17817700 & 3.90321800 & 0.54580900\end{array}$

$\begin{array}{llll}\mathrm{H} & 0.65685600 & 2.75635300 & 1.53010000\end{array}$

$\begin{array}{llll}\text { C } & 4.58610800 & 0.26066000 & -2.00407400\end{array}$

$\begin{array}{lllll}\text { C } & 4.87213500 & 2.63943200 & -1.69539300\end{array}$

$\begin{array}{llll}\text { C } & 3.28083600 & 3.90188000 & -0.27316600\end{array}$

$\begin{array}{llll}\mathrm{H} & 1.80609300 & 4.83751600 & 0.95853900\end{array}$

$\begin{array}{llll}\text { C } & 5.29560100 & 1.45533500 & -2.25372000\end{array}$

$\mathrm{H} \quad 4.91270500 \quad-0.66062200 \quad-2.47751400$

$\mathrm{H} \quad \begin{array}{llll}\mathrm{H} & 5.40159900 & 3.56516100 & -1.90780800\end{array}$

$\mathrm{H} \quad 3.80458800 \quad 4.82508300 \quad-0.50808100$

$\begin{array}{llll}\mathrm{H} & 6.16497000 & 1.43433300 & -2.90430100\end{array}$

C $\quad 3.48563100 \quad-2.61504500 \quad-0.48051100$

$\mathrm{H} \quad 4.17089000 \quad-2.67525800 \quad-1.33811800$

$\begin{array}{llll}\text { C } & 1.40245700 & -1.42230800 & -2.43371500\end{array}$

$\mathrm{H} \quad 1.16488900-0.38745400 \quad-2.71188100$

$\begin{array}{llll}\mathrm{C} & 4.29494400 & -2.31896500 & 0.78406500\end{array}$

$\begin{array}{llll}\mathrm{H} & 3.64232000 & -2.25180000 & 1.66126500\end{array}$

$\begin{array}{llll}\mathrm{H} & 5.00465800 & -3.13489600 & 0.95653700\end{array}$

$\begin{array}{llll}\mathrm{H} & 4.86484100 & -1.38876400 & 0.70485800\end{array}$

C $\quad 2.71118700 \quad-3.92694500 \quad-0.36752400$

$\mathrm{H} \quad 2.20287800 \quad-4.19423500 \quad-1.29680600$

$\mathrm{H} \quad 3.41370000 \quad-4.73255900 \quad-0.12850000$

$\begin{array}{llll}\mathrm{H} & 1.96625800 & -3.88284500 & 0.43424600\end{array}$

$\begin{array}{llll}\mathrm{C} & 0.08891500 & -2.16051400 & -2.17216300\end{array}$

$\mathrm{H} \quad-0.50519000-1.66463500 \quad-1.40230200$

$\mathrm{H} \quad-0.49759300 \quad-2.16840600 \quad-3.09699600$

$\mathrm{H} \quad 0.25027800 \quad-3.19922500-1.87116700$

C $\quad 2.21837000 \quad-2.06086200 \quad-3.56118100$

$\mathrm{H} \quad 2.48206100-3.09813200-3.33458200$

$\mathrm{H} \quad 1.60898100 \quad-2.06538800 \quad-4.47132800$

$\mathrm{H} \quad 3.13336600 \quad-1.50612200 \quad-3.78505300$

$\begin{array}{lll}0.59053500 & 1.37465700 & 2.97798600\end{array}$

$\begin{array}{llll}-2.88689100 & 0.20878900 & -0.08030700\end{array}$

$\begin{array}{lll}-2.96018600 & 1.26465200 & -1.29818100\end{array}$

$\begin{array}{lll}-4.11995300 & -0.71141500 & 0.40550100\end{array}$

$\begin{array}{lll}-1.59288400 & 1.48980700 & -1.77470500\end{array}$

$\begin{array}{llll}-4.02505600 & 1.06458100 & -2.28229100\end{array}$

$\begin{array}{llll}-3.39548200 & 2.84825700 & -0.42095800\end{array}$

$\begin{array}{lll}-3.89978900 & -1.04532500 & 1.81472300\end{array}$

$\begin{array}{llll}-5.45774900 & -0.30070800 & -0.02324900\end{array}$

$\begin{array}{lll}-3.79790100 & -2.31142900 & -0.49139100\end{array}$

$\begin{array}{lll}-2.46989900 & 3.13843200 & 0.49772100\end{array}$

$\begin{array}{lll}-3.44771000 & 3.84554600 & -1.30615000\end{array}$

$\begin{array}{lll}-4.57892400 & 2.74088600 & 0.18271500\end{array}$

$\begin{array}{llll}-2.65750300 & -2.86395800 & -0.06385100\end{array}$

$\begin{array}{llll}-4.79816400 & -3.16147300 & -0.25179000\end{array}$

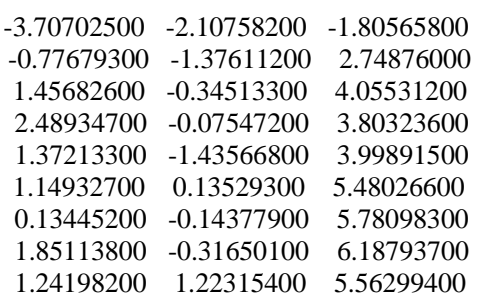

Sum of electronic and zero-point Energies $=$

Sum of electronic and thermal Free Energies=

$-3082.683724$

$-3082.762904$

\section{isomers for cationic complex $c$ is-3b without counter-anion}

\begin{tabular}{|c|c|c|c|}
\hline \multicolumn{4}{|c|}{ cis-3b_cation_no agostic } \\
\hline $\mathrm{P}$ & 1.50740900 & -1.01993000 & 0.07236000 \\
\hline $\mathrm{Au}$ & -0.83345800 & -0.62652000 & -0.35053300 \\
\hline $\mathrm{C}$ & 2.35591600 & -1.86341500 & -1.35241300 \\
\hline $\mathrm{H}$ & 1.80474300 & -2.81310000 & -1.41169000 \\
\hline $\mathrm{C}$ & -0.38646900 & 1.33868300 & -0.16306300 \\
\hline $\mathrm{C}$ & 0.98555200 & 1.67232700 & 0.06739800 \\
\hline $\mathrm{C}$ & -1.35321400 & 2.30913900 & -0.27533700 \\
\hline $\mathrm{C}$ & 2.03455300 & 0.71120600 & 0.20303700 \\
\hline $\mathrm{C}$ & 1.31740100 & 3.06616400 & 0.16430800 \\
\hline $\mathrm{C}$ & -1.00249600 & 3.67629300 & -0.16279700 \\
\hline $\mathrm{H}$ & -2.39033800 & 2.05781700 & -0.45213000 \\
\hline $\mathrm{C}$ & 3.34051100 & 1.12340600 & 0.40002300 \\
\hline $\mathrm{C}$ & 2.66990000 & 3.43901900 & 0.37196300 \\
\hline $\mathrm{C}$ & 0.30090300 & 4.04635700 & 0.04593000 \\
\hline $\mathrm{H}$ & -1.78485700 & 4.42428100 & -0.25282100 \\
\hline $\mathrm{C}$ & 3.66108300 & 2.49403400 & 0.48309800 \\
\hline $\mathrm{H}$ & 4.13524700 & 0.39141800 & 0.49397100 \\
\hline $\mathrm{H}$ & 2.90573300 & 4.49795200 & 0.43943800 \\
\hline $\mathrm{H}$ & 0.57572900 & 5.09463600 & 0.12369800 \\
\hline $\mathrm{H}$ & 4.69309500 & 2.79425000 & 0.63715500 \\
\hline $\mathrm{C}$ & 1.86151500 & -1.96909900 & 1.63997500 \\
\hline $\mathrm{H}$ & 1.94040200 & -3.00499800 & 1.28163400 \\
\hline $\mathrm{C}$ & 3.83883100 & -2.17808900 & -1.15282500 \\
\hline $\mathrm{H}$ & 4.01939700 & -2.80153800 & -0.27313200 \\
\hline $\mathrm{H}$ & 4.20361600 & -2.72950100 & -2.02682900 \\
\hline $\mathrm{H}$ & 4.43791000 & -1.26683500 & -1.06645200 \\
\hline $\mathrm{C}$ & 2.10819200 & -1.07888600 & -2.64343900 \\
\hline $\mathrm{H}$ & 2.63168100 & -0.11733200 & -2.63217600 \\
\hline $\mathrm{H}$ & 2.48451100 & -1.65396100 & -3.49609000 \\
\hline $\mathrm{H}$ & 1.04350000 & -0.88615000 & -2.81333000 \\
\hline $\mathrm{C}$ & 500 & 940400 & 2.58981800 \\
\hline $\mathrm{H}$ & 0.49516800 & -0.83673100 & 2.91442800 \\
\hline $\mathrm{H}$ & -0.25921200 & -2.23724900 & 2.12863100 \\
\hline $\mathrm{H}$ & 0.85358200 & -2.47247200 & 3.48452000 \\
\hline $\mathrm{C}$ & 3.16097600 & -1.58879300 & 2.35080100 \\
\hline $\mathrm{H}$ & 3.10791500 & -0.57387700 & 2.75618400 \\
\hline $\mathrm{H}$ & 3.31923500 & -2.27489900 & 3.19028600 \\
\hline $\mathrm{H}$ & 4.03600700 & -1.65454900 & 1.69985300 \\
\hline $\mathrm{C}$ & -2.84740500 & -0.43495200 & -0.79124600 \\
\hline $\mathrm{H}$ & -3.01410400 & 0.30402200 & -1.58124800 \\
\hline $\mathrm{H}$ & -2.97663200 & -1.43831300 & -1.24187900 \\
\hline $\mathrm{C}$ & -3.79151800 & -0.24888600 & 0.38617500 \\
\hline $\mathrm{H}$ & -3.60648500 & -1.02124900 & 1.14506700 \\
\hline $\mathrm{H}$ & -3.60443600 & 0.71646600 & 0.87598100 \\
\hline $\mathrm{C}$ & -5.26886200 & -0.30576900 & -0.03068600 \\
\hline $\mathrm{H}$ & -5.46918900 & -1.26718100 & -0.52242900 \\
\hline $\mathrm{H}$ & -5.45508200 & 0.46982000 & -0.78561300 \\
\hline $\mathrm{C}$ & -6.22624700 & -0.12084100 & 1.14305300 \\
\hline $\mathrm{H}$ & -6.06793700 & 0.84397700 & 1.63919700 \\
\hline $\mathrm{H}$ & -7.27032600 & -0.15558800 & 0.81346500 \\
\hline $\mathrm{H}$ & -6.09153700 & -0.90602600 & 1.89636500 \\
\hline
\end{tabular}

Sum of electronic and zero-point Energies $=\quad-1255.930421$ Sum of electronic and thermal Free Energies $=\quad-1255.985684$ 


\begin{tabular}{|c|c|c|c|}
\hline \multicolumn{4}{|c|}{$\operatorname{cis}-3 b_{\delta} *$} \\
\hline $\mathrm{Au}$ & 0.99912000 & -0.48370300 & -0.07369100 \\
\hline $\mathrm{C}$ & 2.26480500 & -2.14525200 & 0.02183000 \\
\hline $\mathrm{C}$ & 3.59573400 & -1.77839400 & 0.67705700 \\
\hline $\mathrm{H}$ & 4.23062300 & -2.67715800 & 0.65525200 \\
\hline $\mathrm{H}$ & 3.44105500 & -1.54529500 & 1.73924600 \\
\hline $\mathrm{P}$ & -0.52151600 & 1.40641200 & -0.04611200 \\
\hline $\mathrm{C}$ & -0.69634900 & -1.61313600 & -0.17900900 \\
\hline $\mathrm{C}$ & -1.93941200 & -0.94070100 & 0.05462400 \\
\hline $\mathrm{C}$ & -0.67652500 & -2.95754800 & -0.47165100 \\
\hline $\mathrm{C}$ & -2.05103200 & 0.46994400 & 0.23318000 \\
\hline $\mathrm{C}$ & -3.14032300 & -1.72578600 & 0.08499800 \\
\hline $\mathrm{C}$ & -1.87675800 & -3.70930600 & -0.48197200 \\
\hline $\mathrm{H}$ & 0.24905400 & -3.47581200 & -0.68875900 \\
\hline $\mathrm{C}$ & -3.27145200 & 1.05347500 & 0.51386500 \\
\hline $\mathrm{C}$ & -4.37339500 & -1.08685100 & 0.37566800 \\
\hline $\mathrm{C}$ & -3.07675100 & -3.11539100 & -0.18465100 \\
\hline $\mathrm{H}$ & -1.82642600 & -4.76984900 & -0.71125800 \\
\hline $\mathrm{C}$ & -4.43909800 & 0.26715200 & 0.60366300 \\
\hline $\mathrm{H}$ & -3.34130900 & 2.12704100 & 0.66280300 \\
\hline $\mathrm{H}$ & -5.27293200 & -1.69600400 & 0.41201600 \\
\hline $\mathrm{H}$ & -3.99532300 & -3.69544100 & -0.16244100 \\
\hline $\mathrm{H}$ & -5.38889000 & 0.74130400 & 0.83217500 \\
\hline $\mathrm{C}$ & -0.37079200 & 2.63263900 & 1.33992400 \\
\hline $\mathrm{H}$ & -1.29021200 & 3.23190600 & 1.29808200 \\
\hline $\mathrm{C}$ & -0.74017500 & 2.26604800 & -1.69104400 \\
\hline $\mathrm{H}$ & -1.26314600 & 1.49794500 & -2.27650800 \\
\hline $\mathrm{C}$ & -0.31969000 & 1.89677500 & 2.68158000 \\
\hline $\mathrm{H}$ & 0.58135400 & 1.27872700 & 2.76438600 \\
\hline $\mathrm{H}$ & -0.29521400 & 2.63081900 & 3.49423500 \\
\hline $\mathrm{H}$ & -1.19109700 & 1.25455500 & 2.83761700 \\
\hline $\mathrm{C}$ & 0.83474100 & 3.55742900 & 1.16117700 \\
\hline $\mathrm{H}$ & 0.77525100 & 4.15107900 & 0.24577000 \\
\hline $\mathrm{H}$ & 0.87966600 & 4.25443700 & 2.00502200 \\
\hline $\mathrm{H}$ & 1.77519400 & 2.99594600 & 1.14601600 \\
\hline $\mathrm{C}$ & 0.60447400 & 2.53953600 & -2.36991200 \\
\hline $\mathrm{H}$ & 1.18284200 & 1.62002300 & -2.50396000 \\
\hline $\mathrm{H}$ & 0.42647300 & 2.96586500 & -3.36332200 \\
\hline $\mathrm{H}$ & 1.21630500 & 3.25300600 & -1.81012900 \\
\hline $\mathrm{C}$ & -1.62455900 & 3.51142300 & -1.62441600 \\
\hline $\mathrm{H}$ & -1.15993700 & 4.31198100 & -1.04111800 \\
\hline $\mathrm{H}$ & -1.78271800 & 3.89130600 & -2.63988500 \\
\hline $\mathrm{H}$ & -2.60833300 & 3.29697400 & -1.19821400 \\
\hline $\mathrm{H}$ & 2.40048000 & -2.47984900 & -1.01477600 \\
\hline $\mathrm{C}$ & 4.32911900 & -0.62111000 & 0.01032500 \\
\hline $\mathrm{H}$ & 4.50777000 & -0.84591100 & -1.04861800 \\
\hline $\mathrm{H}$ & 5.31366900 & -0.48876700 & 0.47486400 \\
\hline $\mathrm{C}$ & 3.56090000 & 0.68225200 & 0.14376800 \\
\hline $\mathrm{H}$ & 2.59351100 & 0.65918500 & -0.44460400 \\
\hline $\mathrm{H}$ & 3.33908600 & 0.92768400 & 1.18596500 \\
\hline $\mathrm{H}$ & 4.06132700 & 1.53967700 & -0.31903500 \\
\hline $\mathrm{H}$ & 1.77336800 & -2.93820800 & 0.58844900 \\
\hline
\end{tabular}

Sum of electronic and zero-point Energies $=\quad-1255.934929$ Sum of electronic and thermal Free Energies $=\quad-1255.987828$ $\begin{array}{lll}-3.43501000 & 2.16845300 & 0.20934200\end{array}$

$\begin{array}{lll}-5.31898500 & -1.68251500 & 0.16997000\end{array}$

$\begin{array}{lll}-3.98923200 & -3.71563200 & 0.07587800\end{array}$

$\begin{array}{lll}-5.48432800 & 0.78593200 & 0.26075900\end{array}$

$\begin{array}{lll}-0.45931000 & 2.52286800 & 1.47019400\end{array}$

$\begin{array}{lll}-1.30308100 & 3.21871600 & 1.37723200\end{array}$

$\begin{array}{lll}-0.57976700 & 2.45064700 & -1.59194400\end{array}$

$\begin{array}{lll}-1.12992500 & 1.79754900 & -2.28182600\end{array}$

$\begin{array}{lll}-0.63587700 & 1.69226400 & 2.74277700\end{array}$

$\begin{array}{lll}0.16814900 & 0.95626000 & 2.85570700\end{array}$

$\begin{array}{lll}-0.60406300 & 2.35561200 & 3.61388000\end{array}$

$\begin{array}{lll}-1.59123600 & 1.16062300 & 2.76268400\end{array}$

$\begin{array}{lll}0.84605100 & 3.32121700 & 1.49764000\end{array}$

$\begin{array}{llll}0.96296700 & 3.96368900 & 0.62144100\end{array}$

$\begin{array}{llll}0.85597100 & 3.96505800 & 2.38383700\end{array}$

$\begin{array}{lll}1.71819200 & 2.66096800 & 1.56063500\end{array}$

$\begin{array}{llll}0.83002900 & 2.65450500 & -2.15500900\end{array}$

$\begin{array}{llll}1.33905100 & 1.70121200 & -2.33229000\end{array}$

$\begin{array}{llll}0.76380600 & 3.17563400 & -3.11644600\end{array}$

$\begin{array}{llll}1.45563400 & 3.26193600 & -1.49393800\end{array}$

$\begin{array}{lll}-1.34071300 & 3.76987200 & -1.45345600\end{array}$

$\begin{array}{llll}-0.83425400 & 4.46266000 & -0.77437200\end{array}$

$\begin{array}{llll}-1.40002100 & 4.25369800 & -2.43467500\end{array}$

$\begin{array}{llll}-2.36416700 & 3.62466100 & -1.09831100\end{array}$

$\begin{array}{llll}1.94620300 & -2.74839500 & -1.14134000\end{array}$

$3.90354200 \quad-0.11207700 \quad 0.06897800$

$\begin{array}{llll}3.04104600 & 0.59748800 & 0.07012000\end{array}$

$\begin{array}{llll}4.70199000 & 0.46617400 & -0.41178400\end{array}$

$\begin{array}{lll}4.28395900 & -0.42917600 & 1.51000900\end{array}$

$\begin{array}{llll}3.49003600 & -0.96992500 & 2.03580900\end{array}$

$\begin{array}{llll}5.18465400 & -1.05331900 & 1.53697900\end{array}$

$\begin{array}{llll}4.49582600 & 0.48393500 & 2.07565600\end{array}$

$\begin{array}{lll}2.37085400 & -2.56374500 & 0.58357000\end{array}$

Sum of electronic and zero-point Energies $=\quad-1255.930516$ Sum of electronic and thermal Free Energies $=-1255.984627$

\begin{tabular}{|c|c|c|c|}
\hline $\operatorname{cis}-3 b_{\beta} *$ & & & \\
\hline $\mathrm{Au}$ & 0.94350100 & -0.29067900 & -0.19586100 \\
\hline $\mathrm{C}$ & 2.61631200 & -1.50064800 & -0.38834600 \\
\hline $\mathrm{C}$ & 3.38963300 & -0.21575200 & -0.35716900 \\
\hline $\mathrm{H}$ & 2.64209500 & 0.68538700 & -0.23658200 \\
\hline $\mathrm{H}$ & 3.82314300 & 0.02906100 & -1.33251100 \\
\hline $\mathrm{P}$ & -0.78580400 & 1.35051800 & -0.01277600 \\
\hline $\mathrm{C}$ & -0.55848000 & -1.65892900 & -0.16747200 \\
\hline $\mathrm{C}$ & -1.88898200 & -1.17339900 & 0.03737500 \\
\hline $\mathrm{C}$ & -0.32901300 & -3.01160900 & -0.30807600 \\
\hline $\mathrm{C}$ & -2.19500900 & 0.21512200 & 0.17511800 \\
\hline $\mathrm{C}$ & -2.96126800 & -2.12143100 & 0.12108300 \\
\hline $\mathrm{C}$ & -1.39901300 & -3.93649500 & -0.24495900 \\
\hline $\mathrm{H}$ & 0.67430500 & -3.39226700 & -0.46302000 \\
\hline $\mathrm{C}$ & -3.49124000 & 0.62911700 & 0.41668900 \\
\hline $\mathrm{C}$ & -4.27874200 & -1.65232800 & 0.35934600 \\
\hline $\mathrm{C}$ & -2.68383900 & -3.50384000 & -0.02931000 \\
\hline $\mathrm{H}$ & -1.18692400 & -4.99580100 & -0.36061200 \\
\hline $\mathrm{C}$ & -4.53919400 & -0.31110500 & 0.51278100 \\
\hline $\mathrm{H}$ & -3.71583300 & 1.68435100 & 0.54067300 \\
\hline $\mathrm{H}$ & -5.08330600 & -2.38047000 & 0.42440700 \\
\hline $\mathrm{H}$ & -3.50591500 & -4.21218900 & 0.03164300 \\
\hline $\mathrm{H}$ & -5.55134900 & 0.03277500 & 0.70399800 \\
\hline $\mathrm{C}$ & -0.77795200 & 2.47095100 & 1.46811700 \\
\hline $\mathrm{H}$ & -1.74375700 & 2.99255700 & 1.44445400 \\
\hline $\mathrm{C}$ & -1.06736400 & 2.31337400 & -1.58694500 \\
\hline $\mathrm{H}$ & -1.50191300 & 1.54913800 & -2.24495600 \\
\hline $\mathrm{C}$ & -0.69009200 & 1.64015600 & 2.75048300 \\
\hline $\mathrm{H}$ & 0.25506400 & 1.08759300 & 2.80321700 \\
\hline $\mathrm{H}$ & -0.73327600 & 2.30836000 & 3.61726600 \\
\hline $\mathrm{H}$ & -1.51125600 & 0.92360000 & 2.83994400 \\
\hline $\mathrm{C}$ & 0.34991600 & 3.50150700 & 1.39147300 \\
\hline $\mathrm{H}$ & 0.25643000 & 4.16063800 & 0.52524100 \\
\hline $\mathrm{H}$ & 0.32601900 & 4.12832300 & 2.28960100 \\
\hline $\mathrm{H}$ & 1.33282500 & 3.01879500 & 1.35221400 \\
\hline $\mathrm{C}$ & 0.25341800 & 2.77729800 & -2.20788400 \\
\hline $\mathrm{H}$ & 0.94335800 & 1.94179700 & -2.36375500 \\
\hline $\mathrm{H}$ & 0.05259600 & 3.23100500 & -3.18457500 \\
\hline $\mathrm{H}$ & 0.75650700 & 3.52879200 & -1.59228700 \\
\hline
\end{tabular}




$\begin{array}{lrrr}\mathrm{C} & -2.06953000 & 3.45956500 & -1.44081100 \\ \mathrm{H} & -1.69732300 & 4.24243300 & -0.77311900 \\ \mathrm{H} & -2.23426700 & 3.91522300 & -2.42363400 \\ \mathrm{H} & -3.04071900 & 3.12051600 & -1.07124900 \\ \mathrm{H} & 2.61779500 & -2.04477800 & -1.33228500 \\ \mathrm{C} & 4.37559600 & -0.02510200 & 0.79784100 \\ \mathrm{H} & 3.86769000 & -0.25753700 & 1.74146000 \\ \mathrm{H} & 4.67634100 & 1.02753600 & 0.84586000 \\ \mathrm{C} & 5.61027000 & -0.90771400 & 0.63623800 \\ \mathrm{H} & 5.34490800 & -1.97005700 & 0.61428300 \\ \mathrm{H} & 6.14733700 & -0.67582300 & -0.29033000 \\ \mathrm{H} & 6.30320500 & -0.75292100 & 1.46940600 \\ \mathrm{H} & 2.74413600 & -2.15260300 & 0.47645700\end{array}$

Sum of electronic and zero-point Energies $=\quad-1255.938179$ Sum of electronic and thermal Free Energies $=\quad-1255.992652$

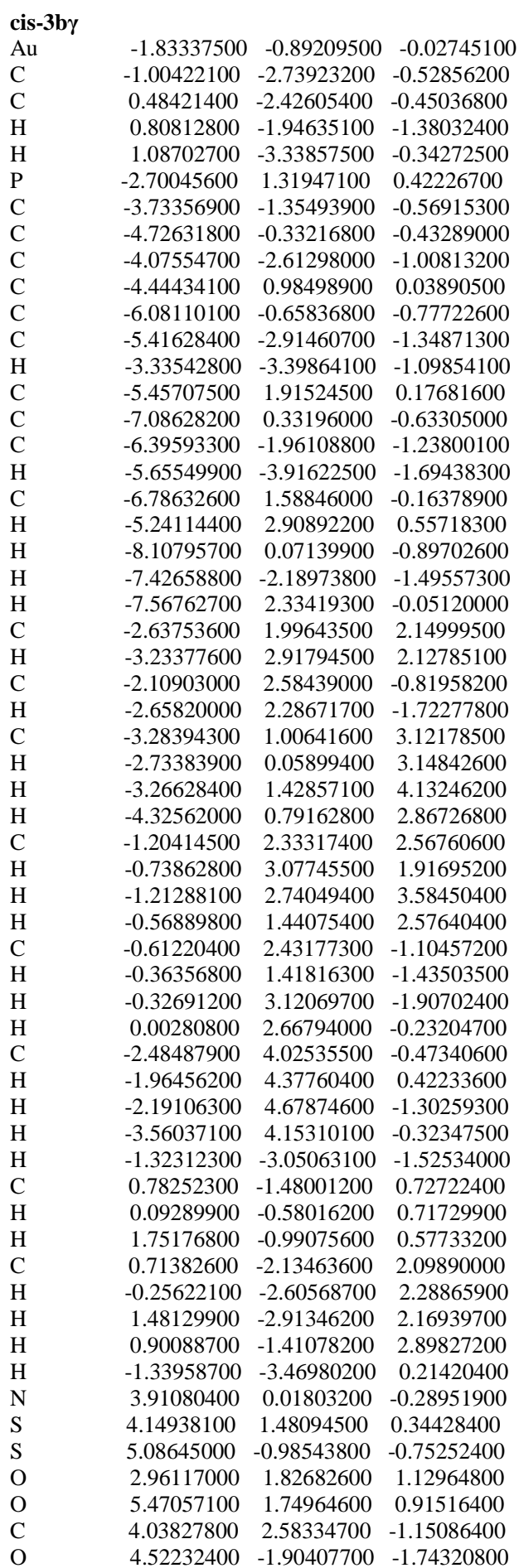

$\begin{array}{lll}6.39853200 & -0.38807800 & -1.00723100\end{array}$

$\begin{array}{lll}5.32825400 & -2.04129500 & 0.76082200\end{array}$

$\begin{array}{lll}2.84288800 & 2.45922200 & -1.73418400\end{array}$

$\begin{array}{llll}4.20131800 & 3.85529900 & -0.77800400\end{array}$

$\begin{array}{lll}4.98239400 & 2.26853900 & -2.03754400\end{array}$

$\begin{array}{lll}4.21084900 & -2.71656700 & 1.04266600\end{array}$

$\begin{array}{lll}6.31053100 & -2.91737500 & 0.53633000\end{array}$

$\begin{array}{lll}5.65164100 & -1.28966700 & 1.81352900\end{array}$

Sum of electronic and zero-point Energies $=\quad-3082.685166$ Sum of electronic and thermal Free Energies $=\quad-3082.764652$

\begin{tabular}{|c|c|c|c|}
\hline ס ס & & & \\
\hline $\mathrm{Au}$ & -1.92134800 & -1.02603300 & 0.27909200 \\
\hline $\mathrm{C}$ & -1.81435200 & -3.09447100 & 0.59234000 \\
\hline $\mathrm{C}$ & -0.82726900 & -3.40547700 & 1.71527600 \\
\hline $\mathrm{H}$ & -0.75161300 & -4.49980500 & 1.80495000 \\
\hline $\mathrm{H}$ & -1.22965200 & -3.04854000 & 2.67294300 \\
\hline $\mathrm{P}$ & -2.17799700 & 1.37653700 & 0.04936100 \\
\hline $\mathrm{C}$ & -3.84694100 & -1.09862500 & -0.37337100 \\
\hline $\mathrm{C}$ & -4.58565700 & 0.12871200 & -0.35879600 \\
\hline $\mathrm{C}$ & -4.44193100 & -2.25670400 & -0.82230500 \\
\hline $\mathrm{C}$ & -3.99344700 & 1.39265800 & -0.06191300 \\
\hline $\mathrm{C}$ & -5.98116900 & 0.09046400 & -0.68790000 \\
\hline $\mathrm{C}$ & -5.80611400 & -2.26741900 & -1.20163700 \\
\hline $\mathrm{H}$ & -3.88880900 & -3.18607800 & -0.88299000 \\
\hline $\mathrm{C}$ & -4.76834000 & 2.53409600 & 0.01046900 \\
\hline $\mathrm{C}$ & -6.74143900 & 1.28532300 & -0.59871500 \\
\hline $\mathrm{C}$ & -6.56770500 & -1.13022100 & -1.10590000 \\
\hline $\mathrm{H}$ & -6.24557000 & -3.19876100 & -1.54743700 \\
\hline $\mathrm{C}$ & -6.15702000 & 2.47525500 & -0.23516600 \\
\hline $\mathrm{H}$ & -4.31459900 & 3.49136900 & 0.24960400 \\
\hline $\mathrm{H}$ & -7.80306500 & 1.24002500 & -0.82792100 \\
\hline $\mathrm{H}$ & -7.62403400 & -1.14448400 & -1.36061200 \\
\hline $\mathrm{H}$ & -6.75268500 & 3.38034500 & -0.16226300 \\
\hline $\mathrm{C}$ & -1.69883900 & 2.49216100 & 1.45312900 \\
\hline $\mathrm{H}$ & -2.15004800 & 3.46366700 & 1.21342800 \\
\hline $\mathrm{C}$ & -1.58737300 & 2.05484900 & -1.59018400 \\
\hline $\mathrm{H}$ & -2.28613400 & 1.57400600 & -2.28813700 \\
\hline $\mathrm{C}$ & -2.30363500 & 1.97577400 & 2.76089800 \\
\hline $\mathrm{H}$ & -1.88122000 & 1.00498300 & 3.04144900 \\
\hline $\mathrm{H}$ & -2.07637000 & 2.68069200 & 3.56798000 \\
\hline $\mathrm{H}$ & -3.39110100 & 1.87242700 & 2.70392800 \\
\hline $\mathrm{C}$ & -0.18328700 & 2.66890000 & 1.55592900 \\
\hline $\mathrm{H}$ & 0.23751700 & 3.13740200 & 0.66436000 \\
\hline $\mathrm{H}$ & 0.04493200 & 3.31717100 & 2.40899700 \\
\hline $\mathrm{H}$ & 0.32934100 & 1.71580500 & 1.71884900 \\
\hline $\mathrm{C}$ & -0.17589600 & 1.57445900 & -1.93634900 \\
\hline $\mathrm{H}$ & -0.10465800 & 0.48293300 & -1.92003400 \\
\hline $\mathrm{H}$ & 0.07701300 & 1.90532500 & -2.94980900 \\
\hline $\mathrm{H}$ & 0.58361200 & 1.97547200 & -1.26030500 \\
\hline $\mathrm{C}$ & -1.74120500 & 3.57002800 & -1.71877400 \\
\hline $\mathrm{H}$ & -1.07170200 & 4.10696100 & -1.03972000 \\
\hline $\mathrm{H}$ & -1.48471200 & 3.87063600 & -2.74076600 \\
\hline $\mathrm{H}$ & -2.76750100 & 3.89794200 & -1.52971100 \\
\hline $\mathrm{H}$ & -1.49104800 & -3.52052400 & -0.36650700 \\
\hline $\mathrm{C}$ & 0.55784700 & -2.80931800 & 1.50094300 \\
\hline $\mathrm{H}$ & 1.01202200 & -3.21805400 & 0.59096300 \\
\hline $\mathrm{H}$ & 1.21727800 & -3.08112200 & 2.33352000 \\
\hline $\mathrm{C}$ & 0.50075800 & -1.29290700 & 1.40820200 \\
\hline $\mathrm{H}$ & 0.02713300 & -0.96004700 & 0.43154600 \\
\hline $\mathrm{H}$ & -0.00129400 & -0.83933300 & 2.26832700 \\
\hline $\mathrm{H}$ & 1.48156000 & -0.81785300 & 1.29284000 \\
\hline $\mathrm{H}$ & -2.80518200 & -3.47944800 & 0.83787700 \\
\hline $\mathrm{N}$ & 3.69152400 & 0.03040100 & 0.07262800 \\
\hline S & 4.64983100 & 1.12340200 & 0.77640700 \\
\hline S & 4.12068500 & -0.87134500 & -1.19544800 \\
\hline $\mathrm{O}$ & 4.14765400 & 1.34055100 & 2.13454600 \\
\hline $\mathrm{O}$ & 6.09050000 & 0.96345800 & 0.57032700 \\
\hline $\mathrm{C}$ & 4.21403100 & 2.69536800 & -0.12102300 \\
\hline $\mathrm{O}$ & 2.89016600 & -1.33502000 & -1.84291000 \\
\hline $\mathrm{O}$ & 5.20610800 & -0.35583000 & -2.03220600 \\
\hline $\mathrm{C}$ & 4.81654900 & -2.40667300 & -0.40275900 \\
\hline F & 2.92609200 & 2.99862500 & 0.07147100 \\
\hline $\mathrm{F}$ & 4.96132300 & 3.69495500 & 0.35304100 \\
\hline F & 4.43277500 & 2.57564400 & -1.42962800 \\
\hline
\end{tabular}


F $\quad 3.87906400 \quad-3.02375200 \quad 0.32052100$

F $\quad \begin{array}{llll}5.23726900 & -3.23985000 & -1.35768900\end{array}$

F $\quad \begin{array}{llll}5.84317400 & -2.10817300 & 0.39225800\end{array}$

Sum of electronic and zero-point Energies $=\quad-3082.690500$

Sum of electronic and thermal Free Energies $=-3082.771130$

\section{(P,C)Au+ $-\mathrm{Au}-\mathrm{Me}+$ ethylene}

\begin{tabular}{|c|c|c|c|}
\hline \multicolumn{4}{|c|}{ cis-3c } \\
\hline $\mathrm{P}$ & 1.40101500 & 1.22390300 & 0.51677000 \\
\hline $\mathrm{Au}$ & 0.35127500 & -0.75280100 & -0.41697700 \\
\hline $\mathrm{C}$ & 0.91432700 & 1.61274900 & 2.27395100 \\
\hline $\mathrm{H}$ & -0.17863200 & 1.54643600 & 2.23170700 \\
\hline $\mathrm{C}$ & 2.27781800 & -1.39722300 & -0.66209200 \\
\hline $\mathrm{C}$ & 3.34874500 & -0.58563700 & -0.14958900 \\
\hline $\mathrm{C}$ & 2.59642100 & -2.59114900 & -1.27820200 \\
\hline $\mathrm{C}$ & 3.13001200 & 0.67916200 & 0.47183600 \\
\hline $\mathrm{C}$ & 4.70202300 & -1.05236500 & -0.26246100 \\
\hline $\mathrm{C}$ & 3.93386300 & -3.03642200 & -1.40121700 \\
\hline $\mathrm{H}$ & 1.82464400 & -3.22995000 & -1.68730100 \\
\hline $\mathrm{C}$ & 4.18515400 & 1.41678800 & 0.97821300 \\
\hline $\mathrm{C}$ & 5.75627200 & -0.26551000 & 0.26759900 \\
\hline $\mathrm{C}$ & 4.96776400 & -2.28962500 & -0.89912400 \\
\hline $\mathrm{H}$ & 4.12691000 & -3.98437300 & -1.89613900 \\
\hline $\mathrm{C}$ & 5.50802500 & 0.93841200 & 0.88233800 \\
\hline $\mathrm{H}$ & 4.00440400 & 2.37488300 & 1.45365800 \\
\hline $\mathrm{H}$ & 6.77329300 & -0.63943600 & 0.17874800 \\
\hline $\mathrm{H}$ & 5.99706100 & -2.62913200 & -0.97985100 \\
\hline $\mathrm{H}$ & 6.32397800 & 1.52856500 & 1.28905100 \\
\hline $\mathrm{C}$ & 1.24689400 & 2.70381300 & -0.61262700 \\
\hline $\mathrm{H}$ & 0.22722200 & 3.06351700 & -0.42822200 \\
\hline $\mathrm{C}$ & 1.28710500 & 2.98894200 & 2.82587600 \\
\hline $\mathrm{H}$ & 0.83278500 & 3.80370300 & 2.25633500 \\
\hline $\mathrm{H}$ & 0.90932900 & 3.06221900 & 3.85253300 \\
\hline $\mathrm{H}$ & 2.36895100 & 3.14650100 & 2.86648600 \\
\hline $\mathrm{C}$ & 1.41532700 & 0.48616200 & 3.18325400 \\
\hline $\mathrm{H}$ & 2.50108900 & & 3.31468700 \\
\hline $\mathrm{H}$ & 0.95074800 & 0.58684900 & 4.16978600 \\
\hline $\mathrm{H}$ & 1.15765700 & -0.50441100 & 2.79708000 \\
\hline $\mathrm{C}$ & 1.35486000 & 2.23578300 & -2.06873500 \\
\hline $\mathrm{H}$ & 2.35014100 & 1.82847000 & -2.27758900 \\
\hline $\mathrm{H}$ & 0.60915800 & 1.47830900 & -2.32050900 \\
\hline $\mathrm{H}$ & 1.20313700 & 3.09330000 & -2.73399000 \\
\hline $\mathrm{C}$ & 2.23975400 & 3.84182900 & -0.36222100 \\
\hline $\mathrm{H}$ & 3.25981300 & 3.53317200 & -0.61008900 \\
\hline $\mathrm{H}$ & 1.98253500 & 4.67874000 & -1.02164600 \\
\hline $\mathrm{H}$ & 2.23153600 & 4.21585000 & 0.66226200 \\
\hline $\mathrm{C}$ & -0.39750800 & -2.44859600 & -1.37624200 \\
\hline $\mathrm{H}$ & -0.01386100 & -2.45886700 & -2.39986300 \\
\hline $\mathrm{H}$ & -0.09323400 & -3.35453800 & -0.84745500 \\
\hline $\mathrm{H}$ & -1.48662000 & -2.39544000 & -1.40783500 \\
\hline $\mathrm{N}$ & -1.75043900 & -0.12068300 & -0.09198000 \\
\hline $\mathrm{S}$ & -2.55390800 & -0.44916100 & 1.31095900 \\
\hline $\mathrm{S}$ & -2.59553300 & 0.47442700 & -1.39530900 \\
\hline $\mathrm{O}$ & -1.58749400 & -0.41543300 & 2.40276100 \\
\hline $\mathrm{O}$ & -3.79938800 & 0.30858400 & 1.38963800 \\
\hline $\mathrm{C}$ & -3.06239100 & -2.25095800 & 1.23087800 \\
\hline $\mathrm{O}$ & -1.62581200 & 0.56801000 & -2.48240300 \\
\hline $\mathrm{O}$ & -3.88304300 & -0.17338200 & -1.61094800 \\
\hline $\mathrm{C}$ & -2.99695800 & 2.26888100 & -0.93660500 \\
\hline $\mathrm{F}$ & -1.98452500 & -3.02520900 & 1.34184800 \\
\hline $\mathrm{F}$ & -3.87395300 & -2.48405000 & 2.25948400 \\
\hline $\mathrm{F}$ & -3.69392700 & -2.52903500 & 0.09854000 \\
\hline $\mathrm{F}$ & -2.74392300 & 3.02272300 & -2.00206300 \\
\hline $\mathrm{F}$ & -4.27306600 & 2.38355500 & -0.61000800 \\
\hline $\mathrm{F}$ & -2.23761200 & 2.68127300 & 0.07885000 \\
\hline
\end{tabular}

Sum of electronic and zero-point Energies=

Sum of electronic and thermal Free Energies=

$-2964.890202$

$-2964.959528$

\begin{tabular}{|c|c|c|c|}
\hline $\mathrm{H}$ & -0.45104600 & 1.46058100 & -2.85298100 \\
\hline $\mathrm{H}$ & 1.21785800 & 1.40553700 & -3.47693400 \\
\hline $\mathrm{H}$ & 0.79032000 & 2.46969500 & -2.09383100 \\
\hline $\mathrm{Au}$ & 1.20180500 & -0.01040900 & -1.23704600 \\
\hline $\mathrm{C}$ & -0.70745000 & -0.91566200 & -2.31299800 \\
\hline $\mathrm{H}$ & -0.37014300 & -1.00107400 & -3.34248900 \\
\hline $\mathrm{H}$ & -1.49797900 & -0.20516800 & -2.09312200 \\
\hline $\mathrm{C}$ & -0.35554800 & -1.84000700 & -1.37237300 \\
\hline $\mathrm{H}$ & -0.86154700 & -1.84771100 & -0.41148400 \\
\hline $\mathrm{H}$ & 0.26263000 & -2.69403100 & -1.62927600 \\
\hline $\mathrm{C}$ & 3.55979700 & -2.50429200 & -0.41694700 \\
\hline $\mathrm{H}$ & 4.23314300 & -1.72499400 & -0.79843700 \\
\hline $\mathrm{C}$ & 2.81166600 & 1.19905600 & -0.73584200 \\
\hline $\mathrm{C}$ & 3.45885300 & 0.96312100 & 0.52074300 \\
\hline $\mathrm{C}$ & 3.27304700 & 2.23432500 & -1.52321400 \\
\hline $\mathrm{C}$ & 3.19689700 & -0.19998900 & 1.29923300 \\
\hline $\mathrm{C}$ & 4.43772500 & 1.89281300 & 1.00240500 \\
\hline $\mathrm{C}$ & 4.28792300 & 3.11304900 & -1.07424800 \\
\hline $\mathrm{H}$ & 2.85371600 & 2.42184200 & -2.50403200 \\
\hline $\mathrm{C}$ & 3.77700300 & -0.37030200 & 2.54113000 \\
\hline $\mathrm{C}$ & 4.99864000 & 1.69645200 & 2.29105500 \\
\hline $\mathrm{C}$ & 4.83130100 & 2.97527600 & 0.17827800 \\
\hline $\mathrm{H}$ & 4.60924700 & 3.91938100 & -1.72806800 \\
\hline $\mathrm{C}$ & 4.65888000 & 0.60547000 & 3.05498300 \\
\hline $\mathrm{H}$ & 3.57517900 & -1.25794700 & 3.13313200 \\
\hline $\mathrm{H}$ & 5.71139100 & 2.42868000 & 2.66205800 \\
\hline $\mathrm{H}$ & 5.57671400 & 3.67519600 & 0.54636600 \\
\hline $\mathrm{H}$ & 5.09046900 & 0.46965600 & 4.04226900 \\
\hline $\mathrm{C}$ & 1.36423200 & -2.44247200 & 1.73180800 \\
\hline $\mathrm{H}$ & 2.17907700 & -2.66795800 & 2.43287500 \\
\hline $\mathrm{C}$ & 3.03285800 & -3.29293200 & -1.61737100 \\
\hline $\mathrm{H}$ & 2.55479500 & -2.63794700 & -2.35265600 \\
\hline $\mathrm{H}$ & 3.87265500 & -3.79128100 & -2.11436600 \\
\hline $\mathrm{H}$ & 2.31828300 & -4.06817800 & -1.32498900 \\
\hline $\mathrm{C}$ & 4.34294300 & -3.37988600 & 0.56173100 \\
\hline $\mathrm{H}$ & 3.73124200 & -4.19295800 & 0.96354600 \\
\hline $\mathrm{H}$ & 5.18968000 & -3.83305600 & 0.03428000 \\
\hline $\mathrm{H}$ & 4.74847900 & -2.80413200 & 1.39862300 \\
\hline $\mathrm{C}$ & 0.76919000 & -3.77991000 & 1.28669400 \\
\hline $\mathrm{H}$ & -0.10008100 & -3.65713300 & 0.63776400 \\
\hline $\mathrm{H}$ & 1.49675200 & -4.41572200 & 0.77705900 \\
\hline $\mathrm{H}$ & 0.43100000 & -4.31819200 & 2.17898900 \\
\hline $\mathrm{C}$ & 0.33712300 & -1.56505400 & 2.45072100 \\
\hline $\mathrm{H}$ & -0.51466500 & -1.32866700 & 1.80679800 \\
\hline $\mathrm{H}$ & -0.04945000 & -2.10538200 & 3.32161400 \\
\hline $\mathrm{H}$ & 0.77079500 & -0.62623400 & 2.80724600 \\
\hline $\mathrm{N}$ & -3.10576800 & 0.26740500 & -0.05722300 \\
\hline S & -3.30067100 & 1.86871400 & -0.05174900 \\
\hline $\mathrm{O}$ & -2.81302800 & 2.38011400 & -1.33477000 \\
\hline $\mathrm{O}$ & -4.56506200 & 2.37896600 & 0.47930700 \\
\hline $\mathrm{C}$ & -2.01196100 & 2.41943400 & 1.17466700 \\
\hline $\mathrm{F}$ & -0.79128600 & 2.04120200 & 0.77037600 \\
\hline $\mathrm{F}$ & -2.03125400 & 3.74864800 & 1.27752900 \\
\hline $\mathrm{F}$ & -2.24338500 & 1.88688300 & 2.37384500 \\
\hline S & -3.95486700 & -0.75883300 & 0.85356700 \\
\hline $\mathrm{O}$ & -3.15204200 & -1.97622600 & 1.00830000 \\
\hline $\mathrm{O}$ & -4.62319500 & -0.19964000 & 2.02964600 \\
\hline $\mathrm{C}$ & -5.33440600 & -1.27245100 & -0.28623800 \\
\hline $\mathrm{F}$ & -4.84191400 & -1.89179700 & -1.36033200 \\
\hline $\mathrm{F}$ & -6.14605200 & -2.11239200 & 0.36010300 \\
\hline $\mathrm{F}$ & -6.03684300 & -0.21219500 & -0.68444800 \\
\hline
\end{tabular}

Sum of electronic and zero-point Energies= Sum of electronic and thermal Free Energies=

$-3043.392533$ $-3043.471691$

TSins(cis-3c $\boldsymbol{\pi} \rightarrow$ trans-3a)
$\begin{array}{lccl}\mathrm{P} & 2.86735300 & -0.93921200 & 0.77517900 \\ \mathrm{C} & -0.01482800 & -0.56027600 & -2.82065700 \\ \mathrm{H} & -0.78280700 & -1.01838100 & -3.43652700 \\ \mathrm{H} & 0.86535300 & -0.41712600 & -3.45043400 \\ \mathrm{H} & -0.43149100 & 0.36799300 & -2.42950300 \\ \mathrm{Au} & 1.32418400 & -0.80163800 & -0.94998100 \\ \mathrm{C} & -0.32516700 & -2.36564500 & -1.87630300 \\ \mathrm{H} & -0.06913100 & -2.89486300 & -2.78955000 \\ \mathrm{H} & -1.37834900 & -2.12958600 & -1.74850500\end{array}$




\begin{tabular}{|c|c|c|c|}
\hline $\mathrm{C}$ & 0.41169900 & -2.70943600 & -0.69741100 \\
\hline $\mathrm{H}$ & -0.13877800 & -2.70035400 & 0.24082800 \\
\hline $\mathrm{H}$ & 1.16270500 & -3.49032900 & -0.78626400 \\
\hline $\mathrm{C}$ & 4.40871500 & -1.79033200 & 0.15704300 \\
\hline $\mathrm{H}$ & 4.78891500 & -1.04985600 & -0.55978000 \\
\hline $\mathrm{C}$ & 2.12021400 & 1.13156900 & -1.19832900 \\
\hline $\mathrm{C}$ & 2.94246500 & 1.63672800 & -0.13999200 \\
\hline $\mathrm{C}$ & 1.84515100 & 1.97519200 & -2.25474600 \\
\hline $\mathrm{C}$ & 3.32619500 & 0.80961600 & 0.95683600 \\
\hline $\mathrm{C}$ & 3.40235900 & 2.99286000 & -0.15568000 \\
\hline $\mathrm{C}$ & 2.32279500 & 3.30837600 & -2.29592500 \\
\hline $\mathrm{H}$ & 1.22815800 & 1.64028400 & -3.08580800 \\
\hline $\mathrm{C}$ & 4.06999200 & 1.31079000 & 2.00827600 \\
\hline $\mathrm{C}$ & 4.16737500 & 3.47551800 & 0.93745400 \\
\hline $\mathrm{C}$ & 3.07383500 & 3.81496800 & -1.26322300 \\
\hline $\mathrm{H}$ & 2.07480700 & 3.93469300 & -3.14921400 \\
\hline $\mathrm{C}$ & 4.48197300 & 2.66124000 & 2.00090200 \\
\hline $\mathrm{H}$ & 4.34966100 & 0.67767300 & 2.84507200 \\
\hline $\mathrm{H}$ & 4.49924500 & 4.51085300 & 0.92269100 \\
\hline $\mathrm{H}$ & 3.42339500 & 4.84409700 & -1.27838100 \\
\hline $\mathrm{H}$ & 5.05959000 & 3.04744100 & 2.83558900 \\
\hline $\mathrm{C}$ & 2.34159800 & -1.56283900 & 2.43933900 \\
\hline $\mathrm{H}$ & 3.18574100 & -1.29302200 & 3.08889000 \\
\hline $\mathrm{C}$ & 4.12502600 & -3.08482200 & -0.60618200 \\
\hline $\mathrm{H}$ & 3.44549200 & -2.91481900 & -1.44639700 \\
\hline $\mathrm{H}$ & 5.06742100 & -3.46982700 & -1.01094100 \\
\hline $\mathrm{H}$ & 3.69806600 & -3.86203100 & 0.03395500 \\
\hline $\mathrm{C}$ & 5.45129300 & -1.97816400 & 1.26157500 \\
\hline $\mathrm{H}$ & 5.12677800 & -2.70804700 & 2.00893800 \\
\hline $\mathrm{H}$ & 6.37692500 & -2.35329600 & 0.81177000 \\
\hline $\mathrm{H}$ & 5.69142500 & -1.04084600 & 1.77098100 \\
\hline $\mathrm{C}$ & 2.15001300 & -3.07853900 & 2.50088500 \\
\hline $\mathrm{H}$ & 1.32390300 & -3.40833100 & 1.86641500 \\
\hline $\mathrm{H}$ & 3.04931100 & -3.62893600 & 2.21496100 \\
\hline $\mathrm{H}$ & 1.90523500 & -3.35668500 & 3.53165700 \\
\hline $\mathrm{C}$ & 1.09523000 & -0.81120800 & 2.91144600 \\
\hline $\mathrm{H}$ & 0.22133000 & -1.07012400 & 2.30600500 \\
\hline $\mathrm{H}$ & 0.87757000 & -1.09445000 & 3.94676300 \\
\hline $\mathrm{H}$ & 1.22727700 & 0.27388800 & 2.88297600 \\
\hline $\mathrm{N}$ & -3.92083100 & 0.69420100 & -0.46255700 \\
\hline S & -3.69611000 & 1.93654900 & 0.54274900 \\
\hline $\mathrm{O}$ & -4.25422700 & 3.13142200 & -0.09013900 \\
\hline $\mathrm{O}$ & -3.96424400 & 1.69273300 & 1.96113100 \\
\hline $\mathrm{C}$ & -1.85393000 & 2.19542000 & 0.44793800 \\
\hline $\mathrm{F}$ & -1.48484500 & 2.48395500 & -0.80249000 \\
\hline $\mathrm{F}$ & -1.49981400 & 3.20508300 & 1.24375900 \\
\hline $\mathrm{F}$ & -1.20317700 & 1.09337100 & 0.83969400 \\
\hline S & -3.94997800 & -0.85050500 & -0.00782400 \\
\hline $\mathrm{O}$ & -3.62366900 & -1.66420100 & -1.18466800 \\
\hline $\mathrm{O}$ & -3.30618700 & -1.18078600 & 1.26698700 \\
\hline $\mathrm{C}$ & -5.76123900 & -1.17869100 & 0.27341300 \\
\hline $\mathrm{F}$ & -6.45024800 & -0.98481100 & -0.85052500 \\
\hline $\mathrm{F}$ & -5.91843200 & -2.44659200 & 0.66231200 \\
\hline $\mathrm{F}$ & -6.24163000 & -0.37343300 & 1.22028500 \\
\hline
\end{tabular}

Sum of electronic and zero-point Energies $=\quad-3043.367420$ Sum of electronic and thermal Free Energies $=\quad-3043.445830$

$\begin{array}{lccc}\text { trans-3a } & & & \\ \mathrm{P} & 2.77100400 & -0.98084000 & 0.73596000 \\ \mathrm{C} & -0.40152800 & -2.18938200 & -3.27324900 \\ \mathrm{H} & -1.20047400 & -2.22806500 & -4.02192900 \\ \mathrm{H} & 0.36204200 & -2.92260200 & -3.55873500 \\ \mathrm{H} & 0.05092100 & -1.18935900 & -3.35145400 \\ \mathrm{Au} & 1.20871100 & -0.73761800 & -0.88685500 \\ \mathrm{C} & -0.94487600 & -2.46325800 & -1.86779500 \\ \mathrm{H} & -1.49084800 & -3.41917600 & -1.87670600 \\ \mathrm{H} & -1.68894900 & -1.70114700 & -1.60870500 \\ \mathrm{C} & 0.12999100 & -2.53881800 & -0.77220000 \\ \mathrm{H} & -0.34003100 & -2.66426300 & 0.20769900 \\ \mathrm{H} & 0.79170400 & -3.39648500 & -0.93953800 \\ \mathrm{C} & 4.22864800 & -1.88113500 & 0.01540900 \\ \mathrm{H} & 4.61711900 & -1.13211600 & -0.68710700 \\ \mathrm{C} & 2.15172900 & 1.12418300 & -1.13885600 \\ \mathrm{C} & 2.98279100 & 1.59922000 & -0.08547800 \\ \mathrm{C} & 1.90943100 & 1.96596900 & -2.20746300\end{array}$

\begin{tabular}{ccc}
3.29891600 & 0.73722800 & 0.99883200 \\
3.49877400 & 2.92973200 & -0.08627400 \\
2.43088000 & 3.28536800 & -2.23226100 \\
1.29302700 & 1.63890000 & -3.04313100 \\
4.04268300 & 1.17922000 & 2.07655900 \\
4.26871100 & 3.35628700 & 1.02660600 \\
3.19934000 & 3.76313700 & -1.19481100 \\
2.20737400 & 3.92678200 & -3.08104000 \\
4.52198300 & 2.50891200 & 2.08354600 \\
4.27277000 & 0.52679700 & 2.91307400 \\
4.65223100 & 4.37355300 & 1.03975100 \\
3.58237500 & 4.78019600 & -1.21085600 \\
5.10376800 & 2.85566000 & 2.93243500 \\
2.17294100 & -1.66172500 & 2.34244600 \\
3.02407600 & -1.42303600 & 2.99976100 \\
3.87146200 & -3.14208400 & -0.76961700 \\
3.18289100 & -2.92282900 & -1.58992400 \\
4.79107700 & -3.54912300 & -1.20332900 \\
3.42863800 & -3.91727700 & -0.13920400 \\
5.28395100 & -2.13188700 & 1.09906300 \\
4.94534600 & -2.86122800 & 1.83990300 \\
6.17868600 & -2.53732300 & 0.61519800 \\
5.57919500 & -1.21386300 & 1.61427100 \\
1.95432700 & -3.17302100 & 2.35819900 \\
1.13128400 & -3.46731600 & 1.70395400 \\
2.84866300 & -3.73046300 & 2.07172000 \\
1.68983800 & -3.46943800 & 3.37860300 \\
0.94529900 & -0.88514500 & 2.82272800 \\
0.07311700 & -1.09677100 & 2.19763400 \\
0.70992000 & -1.19639100 & 3.84556700 \\
1.11322900 & 0.19527800 & 2.83150000 \\
-3.00280100 & 0.20154500 & 0.08577600 \\
-3.12336600 & 1.68850900 & -0.52104100 \\
-2.40390400 & 1.70575800 & -1.79839200 \\
-4.43409700 & 2.33551700 & -0.44165900 \\
-2.03023300 & 2.67752100 & 0.61537600 \\
-0.79387600 & 2.16429700 & 0.63451800 \\
-1.96109400 & 3.93680600 & 0.17951700 \\
-2.50942100 & 2.67430100 & 1.85852300 \\
-4.00982700 & -0.43661800 & 1.17270100 \\
-3.28893400 & -1.50312600 & 1.87161500 \\
-4.81278200 & 0.50531700 & 1.95480900 \\
-5.23564100 & -1.34165900 & 0.10434000 \\
-61679800 & -2.25697700 & -0.64365600 \\
\hline & -1.95729900 & 0.88400700 \\
\hline
\end{tabular}

Sum of electronic and zero-point Energies=

Sum of electronic and thermal Free Energies=

$-3043.402570$

$-3043.480536$

$\begin{array}{lccc}\text { trans-3a} \boldsymbol{\beta} & & & \\ \mathrm{P} & -2.66476200 & -1.14413400 & -0.67455100 \\ \mathrm{Au} & -1.10348100 & -0.59033000 & 0.90437400 \\ \mathrm{C} & 0.25597700 & -2.20052900 & 1.01207500 \\ \mathrm{H} & 0.87420600 & -2.27618600 & 0.11958700 \\ \mathrm{H} & -0.19292900 & -3.14858000 & 1.30576800 \\ \mathrm{C} & 0.81578300 & -1.37636900 & 2.11985900 \\ \mathrm{H} & 0.23264300 & -0.32774100 & 2.20642200 \\ \mathrm{H} & 1.79203500 & -0.95309400 & 1.86482900 \\ \mathrm{C} & 0.72270900 & -1.94374700 & 3.52860000 \\ \mathrm{H} & 1.00648500 & -1.20163400 & 4.27985600 \\ \mathrm{H} & -0.28861300 & -2.29636100 & 3.75139900 \\ \mathrm{H} & 1.40575100 & -2.79520200 & 3.61414700 \\ \mathrm{C} & -2.03795100 & -1.72335700 & -2.31308000 \\ \mathrm{H} & -2.93969000 & -1.71381600 & -2.94172600 \\ \mathrm{C} & -3.94691600 & -2.28672100 & 0.04935000 \\ \mathrm{H} & -4.47397200 & -1.61368500 & 0.73811600 \\ \mathrm{C} & -2.18521500 & 1.19127900 & 1.07555800 \\ \mathrm{C} & -3.18242500 & 1.44099600 & 0.07922000 \\ \mathrm{C} & -1.96214200 & 2.17374000 & 2.02525400 \\ \mathrm{C} & -3.48739600 & 0.46010500 & -0.91050900 \\ \mathrm{C} & -3.88925300 & 2.68420700 & 0.04141100 \\ \mathrm{C} & -2.67712000 & 3.39740500 & 2.01232500 \\ \mathrm{H} & -1.21275000 & 2.02794000 & 2.79940600 \\ \mathrm{C} & -4.40222700 & 0.71286100 & -1.91464000 \\ & & & \\ & & & \\ & & & \end{array}$




$\begin{array}{lccc}\mathrm{C} & -4.83218200 & 2.90922300 & -0.99527300 \\ \mathrm{C} & -3.61431700 & 3.65390300 & 1.03938700 \\ \mathrm{H} & -2.46852700 & 4.14293200 & 2.77546200 \\ \mathrm{C} & -5.07296700 & 1.95510500 & -1.95752500 \\ \mathrm{H} & -4.61611300 & -0.03161900 & -2.67597500 \\ \mathrm{H} & -5.35963500 & 3.85967200 & -1.02394900 \\ \mathrm{H} & -4.15090600 & 4.59911700 & 1.01758800 \\ \mathrm{H} & -5.78740900 & 2.14639600 & -2.75280300 \\ \mathrm{C} & -4.93839100 & -2.77261000 & -1.01200000 \\ \mathrm{H} & -5.74179000 & -3.32394600 & -0.51161600 \\ \mathrm{H} & -5.40025400 & -1.94618600 & -1.55900500 \\ \mathrm{H} & -4.46899500 & -3.45061900 & -1.73070500 \\ \mathrm{C} & -3.35157600 & -3.43297700 & 0.86674600 \\ \mathrm{H} & -4.16864200 & -3.99662800 & 1.32992200 \\ \mathrm{H} & -2.77363400 & -4.12823000 & 0.25175200 \\ \mathrm{H} & -2.70654200 & -3.05766200 & 1.66611800 \\ \mathrm{C} & -1.02485200 & -0.72380800 & -2.87409400 \\ \mathrm{H} & -0.74680700 & -1.02992000 & -3.88810100 \\ \mathrm{H} & -1.42574400 & 0.29218700 & -2.92861300 \\ \mathrm{H} & -0.11250700 & -0.70493600 & -2.27003500 \\ \mathrm{C} & -1.47531300 & -3.14366300 & -2.26977800 \\ \mathrm{H} & -2.21363300 & -3.87758000 & -1.93793000 \\ \mathrm{H} & -1.15540100 & -3.42542100 & -3.27862000 \\ \mathrm{H} & -0.60163800 & -3.20752900 & -1.61481600 \\ \mathrm{~N} & 2.93610100 & 0.25467500 & -0.10451300 \\ \mathrm{~S} & 3.15256400 & 1.79356900 & 0.32673800 \\ \mathrm{O} & 2.53624600 & 1.97914600 & 1.64339900 \\ \mathrm{O} & 4.47424200 & 2.37132900 & 0.07919100 \\ \mathrm{C} & 2.00821400 & 2.69080400 & -0.83580600 \\ \mathrm{~F} & 0.74908000 & 2.27027200 & -0.66520200 \\ \mathrm{~F} & 2.05740500 & 4.00008200 & -0.58583400 \\ \mathrm{~F} & 2.35952000 & 2.47680800 & -2.10332400 \\ \mathrm{~S} & 3.87921700 & -0.54886500 & -1.13786300 \\ \mathrm{O} & 3.09510000 & -1.66180600 & -1.68015900 \\ \mathrm{O} & 4.68874400 & 0.25488600 & -2.05479600 \\ \mathrm{C} & 5.10401000 & -1.36803900 & 0.00009500 \\ \mathrm{~F} & 4.47855000 & -2.21801800 & 0.81826700 \\ \mathrm{~F} & 5.99553600 & -2.04750200 & -0.72421500 \\ \mathrm{~F} & 5.74511600 & -0.45907400 & 0.73494500\end{array}$

Sum of electronic and zero-point Energies $=\quad-3043.413588$ Sum of electronic and thermal Free Energies $=\quad-3043.490860$

$\begin{array}{lccc}\text { TS} \boldsymbol{\beta H} & & & \\ \mathrm{P} & 2.55445700 & -1.30784200 & 0.57571500 \\ \mathrm{Au} & 1.09824800 & -0.32142700 & -0.98951700 \\ \mathrm{C} & -0.45614100 & -1.84636100 & -1.25964600 \\ \mathrm{H} & -1.03712100 & -1.87006100 & -0.34137500 \\ \mathrm{H} & -0.00055900 & -2.78229600 & -1.57424900 \\ \mathrm{C} & -0.83119100 & -0.90550800 & -2.25484800 \\ \mathrm{H} & 0.14121300 & 0.38837000 & -2.11262100 \\ \mathrm{H} & -1.64605000 & -0.22944300 & -1.99310700 \\ \mathrm{C} & -0.61467200 & -1.16675200 & -3.71986400 \\ \mathrm{H} & -0.59012400 & -0.24469100 & -4.30503800 \\ \mathrm{H} & 0.29727800 & -1.73880700 & -3.90688400 \\ \mathrm{H} & -1.46696000 & -1.76186400 & -4.07111900 \\ \mathrm{C} & 1.86565700 & -1.99039200 & 2.15499700 \\ \mathrm{H} & 2.74937100 & -2.18639400 & 2.77748100 \\ \mathrm{C} & 3.71875500 & -2.51093200 & -0.25051400 \\ \mathrm{H} & 4.33746000 & -1.83376800 & -0.85435500 \\ \mathrm{C} & 2.39929100 & 1.28430900 & -0.88708600 \\ \mathrm{C} & 3.40354900 & 1.26688800 & 0.13517800 \\ \mathrm{C} & 2.32053900 & 2.39539300 & -1.71005500 \\ \mathrm{C} & 3.57232400 & 0.14221900 & 0.99820300 \\ \mathrm{C} & 4.26217300 & 2.39816400 & 0.32020000 \\ \mathrm{C} & 3.19551200 & 3.49770500 & -1.55385100 \\ \mathrm{H} & 1.56722700 & 2.44395100 & -2.49026600 \\ \mathrm{C} & 4.49378800 & 0.16719000 & 2.02773000 \\ \mathrm{C} & 5.20250000 & 2.38560400 & 1.38268600 \\ \mathrm{C} & 4.13829400 & 3.50740300 & -0.55460400 \\ \mathrm{H} & 3.10100600 & 4.34830500 & -2.22399200 \\ \mathrm{C} & 5.30647100 & 1.30474100 & 2.22704500 \\ \mathrm{H} & 4.60209200 & -0.68384900 & 2.69400100 \\ \mathrm{H} & 5.83861900 & 3.25611200 & 1.52363300 \\ \mathrm{H} & 4.79605600 & 4.36162800 & -0.41543900 \\ \mathrm{H} & 6.02130700 & 1.31372600 & 3.04479400\end{array}$

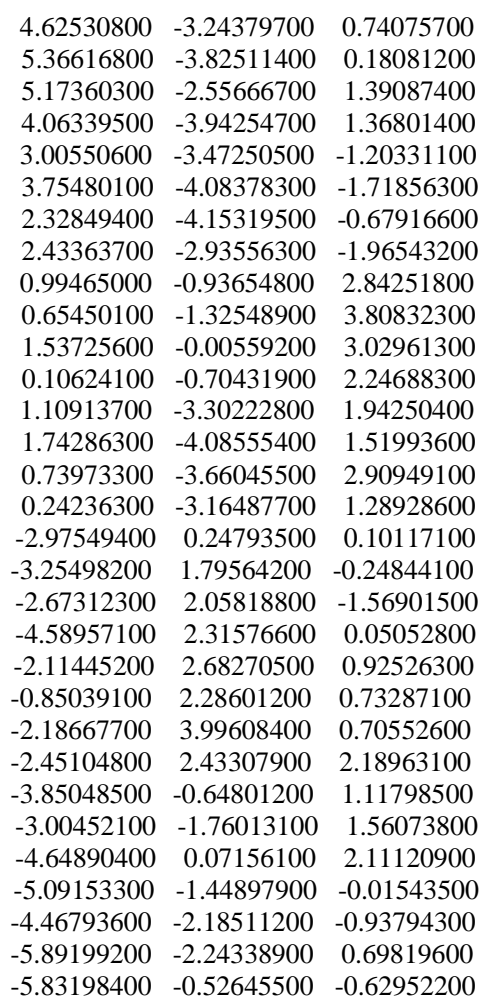

Sum of electronic and zero-point Energies=

Sum of electronic and thermal Free Energies=

$-3043.411194$

$-3043.489886$

cis-3aH

$\begin{array}{lccc}\text { cis-3aH } & & & \\ \mathrm{P} & -2.52526200 & -1.34011500 & -0.48750100 \\ \mathrm{Au} & -1.07973000 & -0.15803700 & 1.03731500 \\ \mathrm{C} & 0.40090000 & -1.93414300 & 1.12013200 \\ \mathrm{H} & 0.93093800 & -1.80836600 & 0.17927000 \\ \mathrm{H} & -0.18845700 & -2.83695600 & 1.25315400 \\ \mathrm{C} & 0.79358000 & -1.19610200 & 2.21512900 \\ \mathrm{H} & -0.34092800 & 0.81666100 & 2.03929700 \\ \mathrm{H} & 1.56948900 & -0.44735000 & 2.06149400 \\ \mathrm{C} & 0.42565500 & -1.51683100 & 3.62666900 \\ \mathrm{H} & 0.23909100 & -0.61500900 & 4.21506500 \\ \mathrm{H} & -0.43752800 & -2.18360500 & 3.69385300 \\ \mathrm{H} & 1.28512800 & -2.02466800 & 4.08467500 \\ \mathrm{C} & -1.87586700 & -2.11063500 & -2.04937800 \\ \mathrm{H} & -2.77411800 & -2.30716700 & -2.64975700 \\ \mathrm{C} & -3.69650700 & -2.49262500 & 0.40726800 \\ \mathrm{H} & -4.30595800 & -1.77921200 & 0.97833100 \\ \mathrm{C} & -2.38904300 & 1.39301800 & 0.75114800 \\ \mathrm{C} & -3.39230100 & 1.26177300 & -0.26428000 \\ \mathrm{C} & -2.30272300 & 2.58231300 & 1.45531900 \\ \mathrm{C} & -3.56527300 & 0.06077000 & -1.01317000 \\ \mathrm{C} & -4.24868200 & 2.37361100 & -0.55741600 \\ \mathrm{C} & -3.17252100 & 3.66447800 & 1.18243700 \\ \mathrm{H} & -1.55081100 & 2.70951500 & 2.22557700 \\ \mathrm{C} & -4.50031600 & -0.01293700 & -2.02804800 \\ \mathrm{C} & -5.20287300 & 2.25596700 & -1.60080600 \\ \mathrm{C} & -4.11802500 & 3.56952200 & 0.19181200 \\ \mathrm{H} & -3.07032500 & 4.58035200 & 1.75818500 \\ \mathrm{C} & -5.31985100 & 1.09606800 & -2.32896500 \\ \mathrm{H} & -4.61483900 & -0.92312700 & -2.60869500 \\ \mathrm{H} & -5.83765100 & 3.11079300 & -1.82066500 \\ \mathrm{H} & -4.77452800 & 4.40529400 & -0.03600400 \\ \mathrm{H} & -6.04589100 & 1.02230500 & -3.13329700 \\ \mathrm{C} & -4.61841100 & -3.27555400 & -0.52916000 \\ \mathrm{H} & -5.34330800 & -3.83486900 & 0.07281400 \\ \mathrm{H} & -5.18530900 & -2.62125400 & -1.19669300 \\ \mathrm{H} & -4.06671100 & -3.99941300 & -1.13678700 \\ \mathrm{C} & -2.98168700 & -3.40165100 & 1.40896500 \\ \mathrm{H} & -3.72920500 & -3.97489800 & 1.96868400 \\ \mathrm{H} & -2.31611400 & -4.11914200 & 0.91980500 \\ \mathrm{H} & -2.39848100 & -2.82287600 & 2.13092300\end{array}$




$\begin{array}{lccc}\mathrm{C} & -0.99527600 & -1.10123500 & -2.79009600 \\ \mathrm{H} & -0.68533700 & -1.52718100 & -3.75055900 \\ \mathrm{H} & -1.52082200 & -0.16407800 & -2.99521300 \\ \mathrm{H} & -0.08826500 & -0.87009100 & -2.22259900 \\ \mathrm{C} & -1.15019200 & -3.43709800 & -1.82097800 \\ \mathrm{H} & -1.77683800 & -4.17820300 & -1.31878800 \\ \mathrm{H} & -0.86131000 & -3.85287900 & -2.79248400 \\ \mathrm{H} & -0.23467800 & -3.30287100 & -1.23978200 \\ \mathrm{~N} & 2.95100800 & 0.20048200 & -0.08015000 \\ \mathrm{~S} & 3.28760800 & 1.70799900 & 0.38231300 \\ \mathrm{O} & 2.70460000 & 1.89867400 & 1.71406700 \\ \mathrm{O} & 4.64709200 & 2.18887700 & 0.13222600 \\ \mathrm{C} & 2.20388300 & 2.73057000 & -0.73541000 \\ \mathrm{~F} & 0.91964600 & 2.38240900 & -0.58890300 \\ \mathrm{~F} & 2.33378700 & 4.01985000 & -0.41907600 \\ \mathrm{~F} & 2.54678000 & 2.55772000 & -2.01054200 \\ \mathrm{~S} & 3.78451800 & -0.62683800 & -1.18718200 \\ \mathrm{O} & 2.90858400 & -1.69137200 & -1.68636600 \\ \mathrm{O} & 4.56300400 & 0.15650500 & -2.14788900 \\ \mathrm{C} & 5.04705000 & -1.53065800 & -0.16120100 \\ \mathrm{~F} & 4.44027000 & -2.30350400 & 0.74230900 \\ \mathrm{~F} & 5.78871600 & -2.30291500 & -0.95824700 \\ \mathrm{~F} & 5.84346200 & -0.67135900 & 0.47308900\end{array}$

Sum of electronic and zero-point Energies=

$-3043.415225$

Sum of electronic and thermal Free Energies

$-3043.495006$

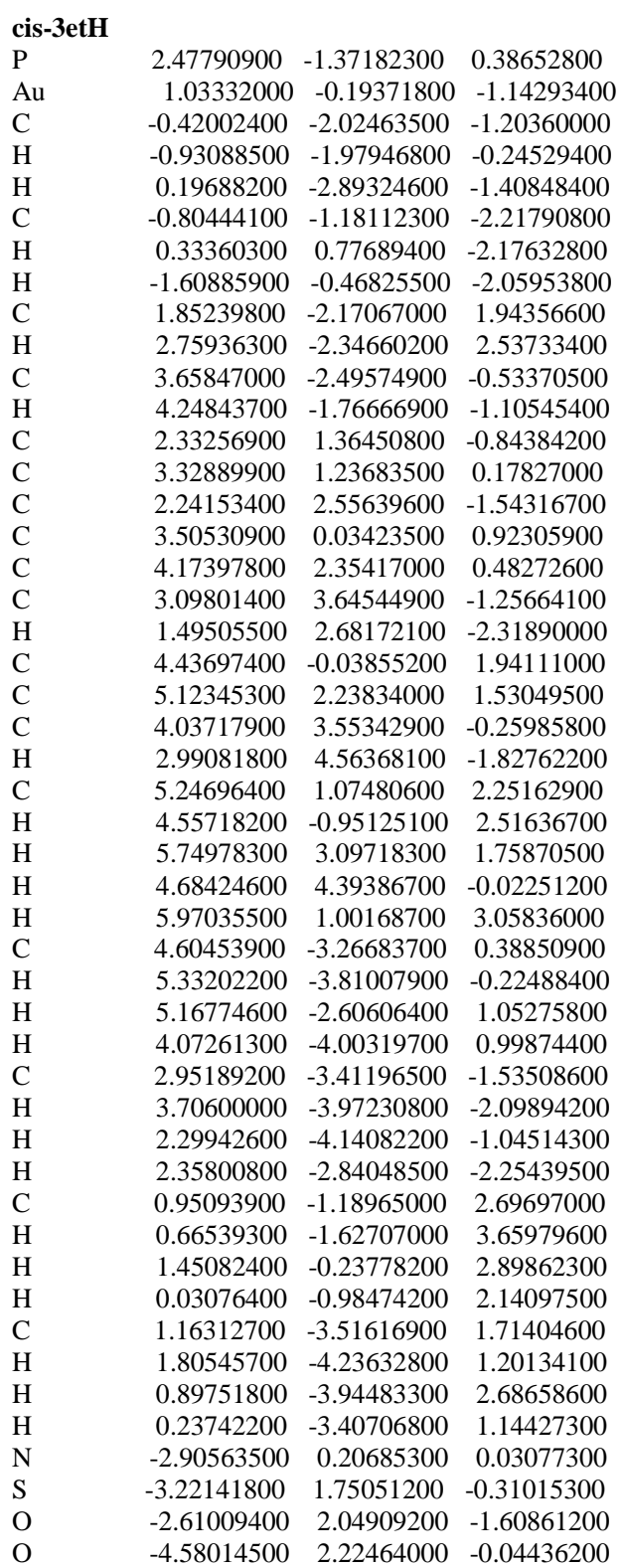

$\begin{array}{llll}\mathrm{C} & -2.14782400 & 2.65568300 & 0.91227600\end{array}$

$\begin{array}{llll}\text { F } & -0.86814900 & 2.28900700 & 0.77104700\end{array}$

$\begin{array}{lllll}\text { F } & -2.24042200 & 3.96853700 & 0.69559200\end{array}$

$\begin{array}{lll}-2.53003600 & 2.39245200 & 2.16108000\end{array}$

$\begin{array}{lll}-3.78386600 & -0.72207200 & 1.01330700\end{array}$

$\begin{array}{lll}-2.91923900 & -1.81447700 & 1.47092900\end{array}$

$\begin{array}{lll}-4.63027200 & -0.03600200 & 1.99022700\end{array}$

$\begin{array}{llll}-4.96602800 & -1.54815200 & -0.16410600\end{array}$

$-4.29103500 \quad-2.25975900-1.06977500$

$\begin{array}{llll}-5.76753500 & -2.36943600 & 0.51713300\end{array}$

$\begin{array}{lll}-5.71089600 & -0.64188200 & -0.79667100\end{array}$

$\begin{array}{lll}-0.46294300 & -1.33912400 & -3.23794400\end{array}$

Sum of electronic and zero-point Energies=

$-3004.132637$

Sum of electronic and thermal Free Energies= $-3004.209240$

\begin{tabular}{|c|c|c|c|}
\hline \multicolumn{4}{|c|}{ TS $\left(\right.$ cis3-et ${ }_{H} \rightarrow$ trans-3d) } \\
\hline $\mathrm{P}$ & 2.59619300 & -1.33723100 & 0.41018500 \\
\hline $\mathrm{Au}$ & 1.08246200 & -0.30780900 & -1.08116600 \\
\hline $\mathrm{C}$ & -0.39089300 & -1.94281800 & -1.39894500 \\
\hline $\mathrm{H}$ & -0.94544100 & -2.05882000 & -0.47166600 \\
\hline $\mathrm{H}$ & 0.14648400 & -2.81083100 & -1.76754200 \\
\hline $\mathrm{C}$ & -0.79968300 & -0.94434400 & -2.30267800 \\
\hline $\mathrm{H}$ & 0.13250900 & 0.44175500 & -2.17061600 \\
\hline $\mathrm{H}$ & -1.65532100 & -0.32032400 & -2.05211000 \\
\hline $\mathrm{C}$ & 1.96522200 & -2.11302000 & 1.97134900 \\
\hline $\mathrm{H}$ & 2.86924900 & -2.30910000 & 2.56390700 \\
\hline $\mathrm{C}$ & 3.77809400 & -2.46509800 & -0.49631500 \\
\hline $\mathrm{H}$ & 4.36534000 & -1.74321500 & -1.07961000 \\
\hline $\mathrm{C}$ & 2.29936500 & 1.34714500 & -0.86867700 \\
\hline $\mathrm{C}$ & 3.32504400 & 1.29991200 & 0.13102600 \\
\hline $\mathrm{C}$ & 2.13024600 & 2.52059100 & -1.58397300 \\
\hline $\mathrm{C}$ & 3.57705900 & 0.11948800 & 0.89280100 \\
\hline $\mathrm{C}$ & 4.11633200 & 2.46304300 & 0.40196600 \\
\hline $\mathrm{C}$ & 2.92943600 & 3.66203800 & -1.33373000 \\
\hline $\mathrm{H}$ & 1.36055000 & 2.58906500 & -2.34608100 \\
\hline $\mathrm{C}$ & 4.52988600 & 0.10806100 & 1.89367500 \\
\hline $\mathrm{C}$ & 5.09220600 & 2.41134200 & 1.43068500 \\
\hline $\mathrm{C}$ & 3.89424900 & 3.64050600 & -0.35630500 \\
\hline $\mathrm{H}$ & 2.76012400 & 4.56455600 & -1.91522300 \\
\hline $\mathrm{C}$ & 5.28979800 & 1.26625700 & 2.16636900 \\
\hline $\mathrm{H}$ & 4.70103700 & -0.78775900 & 2.48371000 \\
\hline $\mathrm{H}$ & 5.68035900 & 3.30289900 & 1.63416600 \\
\hline $\mathrm{H}$ & 4.49673500 & 4.52097400 & -0.14778400 \\
\hline $\mathrm{H}$ & 6.03273800 & 1.24379800 & 2.95837200 \\
\hline $\mathrm{C}$ & 4.72347900 & -3.21687600 & 0.44290100 \\
\hline $\mathrm{H}$ & 5.45777400 & -3.76476900 & -0.15785800 \\
\hline $\mathrm{H}$ & 5.27834200 & -2.54318400 & 1.10127800 \\
\hline $\mathrm{H}$ & 4.19094700 & -3.94703200 & 1.06003600 \\
\hline $\mathrm{C}$ & 3.07098600 & -3.40089100 & -1.47904100 \\
\hline $\mathrm{H}$ & 3.82524700 & -3.97047500 & -2.03328700 \\
\hline $\mathrm{H}$ & 2.42004300 & -4.12002600 & -0.97354100 \\
\hline $\mathrm{H}$ & 2.47314700 & -2.84430900 & -2.20636800 \\
\hline $\mathrm{C}$ & 1.08260400 & -1.11540500 & 2.72487800 \\
\hline $\mathrm{H}$ & 0.77685500 & -1.55531700 & 3.68022000 \\
\hline $\mathrm{H}$ & 1.60475400 & -0.17886600 & 2.94015700 \\
\hline $\mathrm{H}$ & 0.17372800 & -0.88166800 & 2.16140100 \\
\hline $\mathrm{C}$ & 1.24172100 & -3.43608500 & 1.71957600 \\
\hline $\mathrm{H}$ & 1.88707100 & -4.18276300 & 1.25031100 \\
\hline $\mathrm{H}$ & 0.90399200 & -3.84470100 & 2.67811100 \\
\hline $\mathrm{H}$ & 0.35741600 & -3.29576700 & 1.09114900 \\
\hline $\mathrm{N}$ & -2.98345600 & 0.17429400 & -0.02070800 \\
\hline $\mathrm{S}$ & -3.26063900 & 1.73328300 & -0.32147100 \\
\hline $\mathrm{O}$ & -2.65863900 & 2.03756400 & -1.62371200 \\
\hline $\mathrm{O}$ & -4.60196300 & 2.23880300 & -0.02749200 \\
\hline $\mathrm{C}$ & -2.14336600 & 2.58939700 & 0.89744300 \\
\hline $\mathrm{F}$ & -0.87808500 & 2.18477700 & 0.73021900 \\
\hline $\mathrm{F}$ & -2.19882200 & 3.90698400 & 0.69800100 \\
\hline $\mathrm{F}$ & -2.51327500 & 2.32210100 & 2.14848300 \\
\hline $\mathrm{S}$ & -3.84568600 & -0.74006700 & 0.99153700 \\
\hline $\mathrm{O}$ & -3.00181300 & -1.87213800 & 1.38503600 \\
\hline $\mathrm{O}$ & -4.61074100 & -0.03593800 & 2.02160600 \\
\hline $\mathrm{C}$ & -5.12413500 & -1.49841300 & -0.12978100 \\
\hline $\mathrm{F}$ & -4.53270200 & -2.21333200 & -1.08907200 \\
\hline $\mathrm{F}$ & -5.91143100 & -2.30573700 & 0.58425500 \\
\hline
\end{tabular}


F $\quad-5.87287200 \quad-0.55331600 \quad-0.69686400$

$\mathrm{H} \quad-0.54167000 \quad-1.02885500 \quad-3.35597700$

Sum of electronic and zero-point Energies $=\quad-3004.129790$

Sum of electronic and thermal Free Energies $=\quad-3004.206351$

trans-3d

$\begin{array}{llll}\mathrm{P} & 2.71320300 & -1.19361000 & 0.49305800\end{array}$

$\mathrm{Au} \quad 1.09701600 \quad-0.55984500 \quad-0.99160000$

$\begin{array}{llll}\mathrm{C} & -0.20639800 & -2.20115600 & -1.19776100\end{array}$

$\mathrm{H} \quad-0.81020000 \quad-2.36625500 \quad-0.30771500$

$\mathrm{H} \quad 0.26395700 \quad-3.10736500 \quad-1.57292400$

C $\quad-0.81061200 \quad-1.30278000 \quad-2.22368300$

$\mathrm{H} \quad-0.28562400 \quad-0.24362500 \quad-2.28934200$

$\mathrm{H} \quad-1.82155300 \quad-0.96659400 \quad-1.98464100$

$\begin{array}{llll}\mathrm{C} & 2.15313600 & -1.93419900 & 2.09094700\end{array}$

$\mathrm{H} \quad 3.06657200 \quad-1.93054900 \quad 2.70264600$

C $\quad 4.00085600 \quad-2.21607000 \quad-0.38480600$

$\mathrm{H} \quad 4.45763800 \quad-1.46835200 \quad-1.04683900$

C $\quad 2.10707300 \quad 1.27055400 \quad-1.02464600$

$\begin{array}{llll}\text { C } & 3.11418000 & 1.46896700 & -0.02713500\end{array}$

$\begin{array}{llll}\mathrm{C} & 1.82002900 & 2.32875500 & -1.86959300\end{array}$

$\begin{array}{llll}\text { C } & 3.48613700 & 0.41117500 & 0.85411600\end{array}$

$\begin{array}{llll}\mathrm{C} & 3.76718700 & 2.73341800 & 0.11980300\end{array}$

$\begin{array}{llll}\text { C } & 2.47717900 & 3.57848100 & -1.74691600\end{array}$

$\begin{array}{llll}\mathrm{H} & 1.06229600 & 2.22360600 & -2.64222100\end{array}$

$\begin{array}{llll}\text { C } & 4.41529400 & 0.60438400 & 1.85844800\end{array}$

$\begin{array}{llll}\text { C } & 4.72351300 & 2.89887100 & 1.15529800\end{array}$

$\begin{array}{llll}\text { C } & 3.42214400 & 3.78343600 & -0.76926900\end{array}$

$\begin{array}{llll}\mathrm{H} & 2.21620700 & 4.38485800 & -2.42766300\end{array}$

$\begin{array}{llll}\mathrm{C} & 5.03324200 & 1.86535700 & 2.01005000\end{array}$

$\begin{array}{llll}\mathrm{H} & 4.67820400 & -0.20139700 & 2.53726400\end{array}$

$\begin{array}{llll}\mathrm{H} & 5.20840000 & 3.86556000 & 1.26846100\end{array}$

$\begin{array}{lllll}\mathrm{H} & 3.91405100 & 4.74707900 & -0.66279600\end{array}$

$\begin{array}{llll}\mathrm{H} & 5.75950400 & 2.00971700 & 2.80451300\end{array}$

$\begin{array}{lllll}\mathrm{C} & 5.06960500 & -2.72903300 & 0.58341800\end{array}$

$\begin{array}{llll}\mathrm{H} & 5.85552800 & -3.22616600 & 0.00503900\end{array}$

$\mathrm{H} \quad 5.54069600 \quad-1.91987100 \quad 1.14790400$

$\mathrm{H} \quad 4.66391100 \quad-3.45965700 \quad 1.28966000$

C $\quad 3.41418300 \quad-3.32803800 \quad-1.25512100$

$\mathrm{H} \quad 4.23251800 \quad-3.82132700 \quad-1.79071500$

$\mathrm{H} \quad 2.89583800 \quad-4.08939300 \quad-0.66627600$

$\mathrm{H} \quad 2.71819700 \quad-2.93121900 \quad-1.99928100$

$\begin{array}{llll}\text { C } & 1.11035400 & -1.02669800 & 2.74683000\end{array}$

$\mathrm{H} \quad 0.87144900 \quad-1.42002600 \quad 3.74040600$

$\begin{array}{llll}\mathrm{H} & 1.46830200 & -0.00055400 & 2.86887900\end{array}$

$\begin{array}{llll}\mathrm{H} & 0.18341700 & -1.00407400 & 2.16535900\end{array}$

$\begin{array}{llll}\text { C } & 1.65598900 & -3.37201200 & 1.95045400\end{array}$

$2.42904600-4.04643000 \quad 1.57469000$

$\begin{array}{lll}1.34962400 & -3.73426200 & 2.93756000\end{array}$

$\begin{array}{lll}0.78616800 & -3.43412900 & 1.29034500\end{array}$

$\begin{array}{lll}-2.95954100 & 0.17631300 & -0.00130500\end{array}$

$\begin{array}{lll}-3.22323200 & 1.73228200 & -0.33170800\end{array}$

$\begin{array}{llll}-2.58932000 & 2.02520700 & -1.62006300\end{array}$

$\begin{array}{llll}-4.56968300 & 2.24548400 & -0.07531800\end{array}$

$\begin{array}{lll}-2.13490200 & 2.59354300 & 0.90868500\end{array}$

$\begin{array}{lll}-0.86257300 & 2.20538200 & 0.76063100\end{array}$

$\begin{array}{lll}-2.20217800 & 3.91206000 & 0.71698500\end{array}$

$\begin{array}{lll}-2.52299800 & 2.31505500 & 2.15254000\end{array}$

$\begin{array}{lll}-3.86586200 & -0.72462700 & 0.98282800\end{array}$

$\begin{array}{lll}-3.03979300 & -1.84463100 & 1.44245600\end{array}$

$\begin{array}{lll}-4.69088400 & -0.01031900 & 1.95827800\end{array}$

$-5.07520600-1.51006100-0.19461100$

$-4.42610100-2.23507200-1.10917800$

$\begin{array}{lll}-5.89567000 & -2.31379100 & 0.48537000\end{array}$

$-5.79841900-0.58028600-0.81809400$

$-0.69318200 \quad-1.64176800 \quad-3.25475600$

Sum of electronic and zero-point Energies $=\quad-3004.135418$

Sum of electronic and thermal Free Energies $=\quad-3004.210609$

$$
\begin{array}{lccc}
\operatorname{trans3d} \boldsymbol{\pi} & & & \\
\mathrm{P} & 3.45595700 & -1.14170200 & 0.12664100 \\
\mathrm{C} & 0.51235800 & -2.03611700 & 0.41897500 \\
\mathrm{H} & -0.08851300 & -2.14900500 & 1.32230300 \\
\mathrm{H} & 1.29268400 & -2.79460700 & 0.46625900
\end{array}
$$

$\begin{array}{lll}1.41738300 & -0.06025600 & 0.32406700\end{array}$

$\begin{array}{lll}-0.67326800 & 0.28994800 & 1.38612900\end{array}$

$\begin{array}{lll}-0.42812300 & 0.10672000 & 2.42960800\end{array}$

$\begin{array}{lll}-1.39946400 & -0.36979500 & 0.92506700\end{array}$

$\begin{array}{lll}-0.28195400 & 1.44750300 & 0.76942200\end{array}$

$-0.68594800 \quad 1.71147400 \quad-0.20396300$

$\begin{array}{lll}0.23679300 & 2.21975100 & 1.32738300\end{array}$

$\begin{array}{llll}3.52761400 & -2.31927300 & -1.30528000\end{array}$

$2.70964700 \quad-3.02216200 \quad-1.10601500$

$\begin{array}{llll}2.56257700 & 1.67513000 & -0.23012100\end{array}$

$\begin{array}{llll}3.98271000 & 1.50440400 & -0.36026200\end{array}$

$\begin{array}{llll}2.05667100 & 2.92831000 & -0.53337300\end{array}$

$\begin{array}{llll}4.59730600 & 0.23570600 & -0.16520200\end{array}$

$\begin{array}{lll}4.83246800 & 2.60786400 & -0.70223400\end{array}$

$\begin{array}{llll}2.88475500 & 4.01471700 & -0.90940200\end{array}$

$\begin{array}{llll}0.99170000 & 3.12787400 & -0.49159300\end{array}$

$\begin{array}{llll}5.96900500 & 0.07302800 & -0.23432900\end{array}$

$\begin{array}{llll}6.23549100 & 2.41052900 & -0.77021200\end{array}$

$\begin{array}{llll}4.24748100 & 3.86963100 & -0.97346000\end{array}$

$\begin{array}{llll}2.42466100 & 4.97365300 & -1.13398000\end{array}$

$\begin{array}{llll}6.79564500 & 1.17748700 & -0.52850200\end{array}$

$\begin{array}{llll}6.42217300 & -0.89882200 & -0.06397200\end{array}$

$\begin{array}{llll}6.86704000 & 3.26023700 & -1.01825800\end{array}$

$\begin{array}{llll}4.89031400 & 4.70502800 & -1.23878800\end{array}$

$\begin{array}{llll}7.87216100 & 1.04270600 & -0.57517300\end{array}$

$\begin{array}{llll}4.03656300 & -1.95133800 & 1.69482800\end{array}$

$\begin{array}{llll}5.09908800 & -2.13443100 & 1.48177200\end{array}$

$\begin{array}{llll}4.83833400 & -3.10544800 & -1.38810900\end{array}$

$\begin{array}{llll}5.06356400 & -3.65348700 & -0.46921100\end{array}$

$\begin{array}{llll}4.75262700 & -3.83962000 & -2.19670900\end{array}$

$\begin{array}{llll}5.68448000 & -2.45434600 & -1.62570300\end{array}$

$3.23127500 \quad-1.56785300 \quad-2.60594900$

$\begin{array}{llll}4.04125700 & -0.87606900 & -2.85760700\end{array}$

$\begin{array}{llll}3.13879300 & -2.28923700 & -3.42436600\end{array}$

$\begin{array}{llll}2.29889800 & -0.99716400 & -2.55396400\end{array}$

$\begin{array}{llll}3.37178600 & -3.28742100 & 2.02502700\end{array}$

$\begin{array}{lll}2.34127000 & -3.15080500 & 2.36332900\end{array}$

$\begin{array}{lll}3.37387600 & -3.98615600 & 1.18364100\end{array}$

$\begin{array}{llll}3.92622100 & -3.75749700 & 2.84449900\end{array}$

$\begin{array}{lll}3.93287300 & -0.96655300 & 2.86175100\end{array}$

$\begin{array}{lll}2.88646200 & -0.74605500 & 3.09881600\end{array}$

$\begin{array}{lll}4.39007900 & -1.41457200 & 3.75041700\end{array}$

$\begin{array}{llll}4.44867600 & -0.02345500 & 2.65993300\end{array}$

$-0.30991700 \quad-2.21609200 \quad-0.84791400$

$\begin{array}{llll}0.30943500 & -2.17255000 & -1.75041300\end{array}$

$\begin{array}{llll}-0.79458300 & -3.20225000 & -0.83795800\end{array}$

$\begin{array}{lll}-1.10349600 & -1.47124200 & -0.95816600\end{array}$

$\begin{array}{llll}-3.85729100 & 0.09134700 & -0.21950900\end{array}$

$\begin{array}{llll}-4.62244100 & 1.42778600 & -0.70436300\end{array}$

$\begin{array}{llll}-3.76788100 & 2.08892700 & -1.69318300\end{array}$

$\begin{array}{llll}-6.05543300 & 1.30240900 & -0.97695600\end{array}$

$\begin{array}{lll}-4.52179000 & 2.53243700 & 0.79199000\end{array}$

$\begin{array}{lll}-3.24773100 & 2.80295800 & 1.08900400\end{array}$

$\begin{array}{lll}-5.15103000 & 3.68119500 & 0.53258700\end{array}$

$\begin{array}{lll}-5.09343500 & 1.95529200 & 1.84841600\end{array}$

$\begin{array}{lll}-4.58591000 & -1.17525900 & 0.46485200\end{array}$

$\begin{array}{llll}-3.56894600 & -1.90969900 & 1.22375100\end{array}$

$\begin{array}{lll}-5.88940300 & -0.93177600 & 1.08576100\end{array}$

$\begin{array}{llll}-4.94836800 & -2.28031300 & -0.98915400\end{array}$

$\begin{array}{llll}-3.81567200 & -2.62960000 & -1.60296500\end{array}$

$\begin{array}{llll}-5.56062900 & -3.38631000 & -0.55849600\end{array}$

$\begin{array}{llll}-5.73862600 & -1.66296200 & -1.86655300\end{array}$

Sum of electronic and zero-point Energies $=\quad-3082.658831$ Sum of electronic and thermal Free Energies=

$-3082.740543$

$\begin{array}{lccc}\text { TS(trans-3d } \boldsymbol{\pi} \rightarrow \mathbf{c i s}-\mathbf{3 b}) & & \\ \mathrm{P} & 3.41682100 & -1.25271000 & 0.17263500 \\ \mathrm{C} & -0.04671100 & -1.64360200 & 0.53799100 \\ \mathrm{H} & -0.82816200 & -1.84884800 & 1.26508000 \\ \mathrm{H} & 0.81083000 & -2.22645500 & 0.87000600 \\ \mathrm{Au} & 1.50865300 & 0.17244400 & 0.30676100 \\ \mathrm{C} & -0.79183400 & 0.30184100 & 1.05942100 \\ \mathrm{H} & -0.82325700 & 0.12931700 & 2.13169500 \\ \mathrm{H} & -1.66473200 & -0.03549000 & 0.50992000 \\ \mathrm{C} & -0.11298200 & 1.47214400 & 0.59583100\end{array}$




\begin{tabular}{|c|c|c|c|}
\hline $\mathrm{H}$ & -0.43250200 & 1.89494600 & -0.35478200 \\
\hline $\mathrm{H}$ & 0.21138600 & 2.19446800 & 1.34277800 \\
\hline $\mathrm{C}$ & 3.33124700 & -2.52322100 & -1.18407300 \\
\hline $\mathrm{H}$ & 2.42035100 & -3.08729100 & -0.94050800 \\
\hline$C$ & 2.84488700 & 1.66855000 & -0.20698300 \\
\hline $\mathrm{C}$ & 4.22220700 & 1.31472000 & -0.39321300 \\
\hline 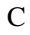 & 2.47470500 & 2.98690200 & -0.39562500 \\
\hline $\mathrm{C}$ & 4.69057100 & -0.02312700 & -0.23028400 \\
\hline $\mathrm{C}$ & 5.17736700 & 2.32446900 & -0.74531400 \\
\hline $\mathrm{C}$ & 3.41584000 & 3.97991800 & -0.76061100 \\
\hline $\mathrm{H}$ & 1.44324900 & 3.29788100 & -0.26783100 \\
\hline $\mathrm{C}$ & 6.03183900 & -0.33075600 & -0.36540300 \\
\hline $\mathrm{C}$ & 6.54234100 & 1.96751900 & -0.89407600 \\
\hline $\mathrm{C}$ & 4.74005000 & 3.66031500 & -0.92795200 \\
\hline $\mathrm{H}$ & 3.07653000 & 5.00291400 & -0.90075200 \\
\hline $\mathrm{C}$ & 6.96479400 & 0.67321100 & -0.70030000 \\
\hline $\mathrm{H}$ & 6.38205200 & -1.34684900 & -0.21122000 \\
\hline $\mathrm{H}$ & 7.25462300 & 2.74509600 & -1.15877300 \\
\hline $\mathrm{H}$ & 5.46929700 & 4.41944500 & -1.19874900 \\
\hline $\mathrm{H}$ & 8.01457900 & 0.41537700 & -0.80560900 \\
\hline $\mathrm{C}$ & 3.98088500 & -2.05857600 & 1.75097200 \\
\hline $\mathrm{H}$ & 4.98975700 & -2.42531800 & 1.51897700 \\
\hline $\mathrm{C}$ & 4.50973900 & -3.49771200 & -1.23052800 \\
\hline $\mathrm{H}$ & 4.67230100 & -4.01410700 & -0.28066700 \\
\hline $\mathrm{H}$ & 4.30721200 & -4.26187500 & -1.98946300 \\
\hline $\mathrm{H}$ & 5.43756900 & -2.99322700 & -1.51580000 \\
\hline $\mathrm{C}$ & 3.12893300 & -1.82237100 & -2.53078200 \\
\hline $\mathrm{H}$ & 4.03233900 & -1.28370600 & -2.83432500 \\
\hline $\mathrm{H}$ & 2.90806600 & -2.56875000 & -3.30108400 \\
\hline $\mathrm{H}$ & 2.30090100 & -1.10643000 & -2.51059600 \\
\hline $\mathrm{C}$ & 3.10792100 & -3.24175300 & 2.17444800 \\
\hline $\mathrm{H}$ & 2.11434700 & -2.91144200 & 2.49359600 \\
\hline $\mathrm{H}$ & 2.98846500 & -3.98982900 & 1.38542300 \\
\hline $\mathrm{H}$ & 3.57514200 & -3.73963500 & 3.03116000 \\
\hline $\mathrm{C}$ & 4.08196300 & -1.01454800 & 2.86536800 \\
\hline $\mathrm{H}$ & 3.09931700 & -0.59458600 & 3.10767600 \\
\hline $\mathrm{H}$ & 4.47386200 & -1.48928700 & 3.77149700 \\
\hline $\mathrm{H}$ & 4.75184100 & -0.19056500 & 2.60367500 \\
\hline $\mathrm{C}$ & -0.47700400 & -1.96981100 & -0.87514800 \\
\hline $\mathrm{H}$ & 0.32316500 & -1.83156000 & -1.60730300 \\
\hline $\mathrm{H}$ & -0.78530300 & -3.02317900 & -0.91864500 \\
\hline $\mathrm{H}$ & -1.33109400 & -1.36839100 & -1.19765000 \\
\hline $\mathrm{N}$ & -3.97360000 & 0.07259400 & -0.24227600 \\
\hline S & -4.71739200 & 1.41809200 & -0.73656800 \\
\hline $\mathrm{O}$ & -3.88019100 & 2.02831300 & -1.77135500 \\
\hline $\mathrm{O}$ & -6.16179300 & 1.32537800 & -0.95515400 \\
\hline $\mathrm{C}$ & -4.53098600 & 2.55639000 & 0.72585300 \\
\hline $\mathrm{F}$ & -3.23952000 & 2.78829100 & 0.97669600 \\
\hline $\mathrm{F}$ & -5.12787000 & 3.71937300 & 0.45515300 \\
\hline $\mathrm{F}$ & -5.08902800 & 2.02715500 & 1.81439800 \\
\hline S & -4.70851600 & -1.15854100 & 0.49773900 \\
\hline $\mathrm{O}$ & -3.68723600 & -1.88783500 & 1.25579300 \\
\hline $\mathrm{O}$ & -5.99282000 & -0.87272100 & 1.13960900 \\
\hline $\mathrm{C}$ & -5.12277500 & -2.29581900 & -0.91680500 \\
\hline $\mathrm{F}$ & -4.00902000 & -2.69707000 & -1.53317400 \\
\hline $\mathrm{F}$ & -5.76204000 & -3.36931200 & -0.44566300 \\
\hline $\mathrm{F}$ & -5.90585000 & -1.68057700 & -1.80207600 \\
\hline
\end{tabular}

Sum of electronic and zero-point Energies $=\quad-3082.640026$ Sum of electronic and thermal Free Energies $=\quad-3082.719937$

$\begin{array}{lrrr}\text { cis-3b } & & & \\ \mathrm{Au} & 1.11008400 & -0.10217600 & 0.91758800 \\ \mathrm{C} & -0.06877300 & 0.63164000 & 2.45265400 \\ \mathrm{C} & -0.62259400 & -0.75954400 & 2.50180700 \\ \mathrm{H} & -0.16277800 & -1.40551300 & 1.62297200 \\ \mathrm{H} & -1.67397400 & -0.79301300 & 2.19596400 \\ \mathrm{P} & 2.39335100 & -1.26350400 & -0.73717200 \\ \mathrm{C} & 2.33539400 & 1.47388400 & 0.53233000 \\ \mathrm{C} & 3.46783100 & 1.23592600 & -0.30980200 \\ \mathrm{C} & 2.14221200 & 2.72043600 & 1.09013400 \\ \mathrm{C} & 3.70328200 & -0.01815300 & -0.95266600 \\ \mathrm{C} & 4.41488400 & 2.29364500 & -0.50962700 \\ \mathrm{C} & 3.06417100 & 3.76948500 & 0.85599100 \\ \mathrm{H} & 1.28403600 & 2.91720200 & 1.72326700\end{array}$

\begin{tabular}{|c|c|c|}
\hline & & \\
\hline 006300 & 2.04553300 & \\
\hline & & \\
\hline & & \\
\hline 33800 & 0.81542000 & \\
\hline & & \\
\hline & 300 & \\
\hline & & \\
\hline & & \\
\hline & -2.8 & \\
\hline & & \\
\hline & -1.4 & \\
\hline & -0.4 & \\
\hline & & \\
\hline & -2.3 & \\
\hline & -3 & \\
\hline & & \\
\hline & & \\
\hline & & \\
\hline & & \\
\hline & -3 . & \\
\hline & -1 & \\
\hline & & \\
\hline & & \\
\hline & & \\
\hline & -2. & \\
\hline 0 & -3 & \\
\hline & -2 & \\
\hline & -1 & \\
\hline & & \\
\hline & & \\
\hline & & \\
\hline & & \\
\hline & & \\
\hline & -0 . & \\
\hline & & \\
\hline & -1 & \\
\hline & & \\
\hline & & \\
\hline & 1.4 & -1 . \\
\hline & -0.5 & \\
\hline & & \\
\hline-3.7 & & -2 \\
\hline & & \\
\hline & -1. & \\
\hline & -0.0 & \\
\hline & -2.1 & -0 \\
\hline & 3.2 & 0.5 \\
\hline & 4.0 & \\
\hline & & \\
\hline & -2.7 & 18300 \\
\hline & & -0.7 \\
\hline & -1.83830 & \\
\hline
\end{tabular}

Sum of electronic and zero-point Energies= Sum of electronic and thermal Free Energies=

$-3082.697542$ 3082.778554

\section{Reductive elimination process}

\begin{tabular}{lccc}
\multicolumn{4}{l}{ cis-3H_cation } \\
$\mathrm{Au}$ & -0.23824200 & -1.59293600 & -0.00678600 \\
$\mathrm{P}$ & -1.10596900 & 0.65497400 & 0.01874100 \\
$\mathrm{C}$ & 1.58611100 & -0.75268100 & 0.08568500 \\
$\mathrm{C}$ & 1.64123200 & 0.67359000 & 0.05577500 \\
$\mathrm{C}$ & 2.71815800 & -1.53108600 & 0.15411400 \\
$\mathrm{C}$ & 0.49326100 & 1.51716100 & -0.00588700 \\
$\mathrm{C}$ & 2.94257700 & 1.27794000 & 0.08166600 \\
$\mathrm{C}$ & 3.98836600 & -0.90641900 & 0.19364300 \\
$\mathrm{H}$ & 2.65781700 & -2.61123700 & 0.17412900 \\
$\mathrm{C}$ & 0.63572100 & 2.88987600 & -0.06984700 \\
$\mathrm{C}$ & 3.03927500 & 2.69194300 & 0.02364800 \\
$\mathrm{C}$ & 4.09816400 & 0.46041500 & 0.15301300
\end{tabular}




$\begin{array}{lrrr}\mathrm{H} & 4.87366000 & -1.53292600 & 0.24866700 \\ \mathrm{C} & 1.91670100 & 3.48117000 & -0.05637400 \\ \mathrm{H} & -0.24075200 & 3.52745300 & -0.13534300 \\ \mathrm{H} & 4.02919300 & 3.14005100 & 0.03767900 \\ \mathrm{H} & 5.07366100 & 0.93790300 & 0.17290900 \\ \mathrm{H} & 2.00831900 & 4.56167500 & -0.10875900 \\ \mathrm{C} & -2.07508400 & 1.21831100 & -1.46016700 \\ \mathrm{H} & -2.18783400 & 2.30404100 & -1.34144500 \\ \mathrm{C} & -1.95690100 & 1.08734700 & 1.61948300 \\ \mathrm{H} & -1.11359200 & 1.11036800 & 2.32263500 \\ \mathrm{C} & -1.28161600 & 0.93580900 & -2.73814900 \\ \mathrm{H} & -1.13035400 & -0.13930800 & -2.88659700 \\ \mathrm{H} & -1.84212100 & 1.31436100 & -3.59963000 \\ \mathrm{H} & -0.30302100 & 1.42393300 & -2.73668100 \\ \mathrm{C} & -3.46145000 & 0.57229200 & -1.50082700 \\ \mathrm{H} & -4.07486200 & 0.84451400 & -0.63821400 \\ \mathrm{H} & -3.98627200 & 0.91056700 & -2.40072600 \\ \mathrm{H} & -3.39668000 & -0.52065900 & -1.54869000 \\ \mathrm{C} & -2.90832700 & -0.02968100 & 2.05917200 \\ \mathrm{H} & -2.40411200 & -1.00197000 & 2.09561500 \\ \mathrm{H} & -3.27795700 & 0.18916100 & 3.06674500 \\ \mathrm{H} & -3.77758500 & -0.11696600 & 1.40071700 \\ \mathrm{C} & -2.62980000 & 2.45996200 & 1.61236300 \\ \mathrm{H} & -3.45867000 & 2.50181700 & 0.89953000 \\ \mathrm{H} & -3.03821000 & 2.66288000 & 2.60851100 \\ \mathrm{H} & -1.92773700 & 3.26407500 & 1.37619000 \\ \mathrm{H} & 0.44943900 & -3.02806600 & 0.00388700\end{array}$

Sum of electronic and zero-point Energies $=\quad-1098.829837$ Sum of electronic and thermal Free Energies $=\quad-1098.877296$

\section{TSRE-noNTf2}

$\begin{array}{lccc}\mathrm{Au} & 0.13500600 & -1.58573800 & -0.07665700 \\ \mathrm{P} & 1.22297100 & 0.49953100 & 0.06909700 \\ \mathrm{C} & -0.39162800 & 1.36538700 & -0.18267900 \\ \mathrm{C} & -1.63962600 & 0.64835100 & -0.06816800 \\ \mathrm{C} & -0.40298000 & 2.73536700 & -0.38135900 \\ \mathrm{C} & -1.79902800 & -0.78557900 & 0.06407300 \\ \mathrm{C} & -2.84863500 & 1.42497000 & -0.02056600 \\ \mathrm{C} & -1.60371800 & 3.46959900 & -0.43794900 \\ \mathrm{H} & 0.53574200 & 3.27063500 & -0.48550600 \\ \mathrm{C} & -3.02419100 & -1.34605200 & 0.36960400 \\ \mathrm{H} & -1.30780800 & -2.16756100 & -0.52151800 \\ \mathrm{C} & -4.09546400 & 0.80608000 & 0.25884400 \\ \mathrm{C} & -2.79714000 & 2.82725200 & -0.22737900 \\ \mathrm{H} & -1.57269000 & 4.54110900 & -0.60993400 \\ \mathrm{C} & -4.17760600 & -0.54028300 & 0.49444600 \\ \mathrm{H} & -3.12345000 & -2.42044700 & 0.48077400 \\ \mathrm{H} & -4.98335100 & 1.43142600 & 0.29437700 \\ \mathrm{H} & -3.73108400 & 3.38262800 & -0.21014000 \\ \mathrm{H} & -5.12715600 & -1.01012100 & 0.73154400 \\ \mathrm{C} & 2.35695200 & 1.04015600 & -1.29714900 \\ \mathrm{H} & 2.46467500 & 2.12783400 & -1.19795900 \\ \mathrm{C} & 3.73220900 & 0.38522800 & -1.14614200 \\ \mathrm{H} & 4.37104300 & 0.69244800 & -1.98138300 \\ \mathrm{H} & 4.23481400 & 0.67686800 & -0.22060600 \\ \mathrm{H} & 3.66003900 & -0.70821500 & -1.16829500 \\ \mathrm{C} & 1.73048900 & 0.71959200 & -2.65559000 \\ \mathrm{H} & 1.58706300 & -0.35959400 & -2.78410400 \\ \mathrm{H} & 0.76472300 & 1.21329300 & -2.79549100 \\ \mathrm{H} & 2.39992000 & 1.06227700 & -3.45201500 \\ \mathrm{C} & 1.85020500 & 1.06580900 & 1.73800700 \\ \mathrm{H} & 0.92095900 & 1.14210200 & 2.31760500 \\ \mathrm{C} & 2.72486200 & -0.01367800 & 2.38210600 \\ \mathrm{H} & 2.98603500 & 0.29357500 & 3.40076100 \\ \mathrm{H} & 2.20209600 & -0.97352900 & 2.44507800 \\ \mathrm{H} & 3.65881200 & -0.16770100 & 1.83325100 \\ \mathrm{C} & 2.53554200 & 2.43222800 & 1.72760000 \\ \mathrm{H} & 1.89160700 & 3.22068200 & 1.32932200 \\ \mathrm{H} & 2.79114200 & 2.70865200 & 2.75664900 \\ \mathrm{H} & 3.46536700 & 2.41737900 & 1.15086400\end{array}$

Sum of electronic and zero-point Energies= Sum of electronic and thermal Free Energies=

$\begin{array}{lccc}\text { Cationic gold(I)_noNTf2 } & & \\ \text { Au } & 1.25733300 & -1.31966600 & 0.50400500 \\ \mathrm{P} & 0.66270400 & 0.74154400 & -0.20136100 \\ \mathrm{C} & -1.11865700 & 1.08949700 & 0.10972200 \\ \mathrm{C} & -2.16727800 & 0.11328200 & -0.05337100 \\ \mathrm{C} & -1.44508700 & 2.36318200 & 0.55045300 \\ \mathrm{C} & -1.97322400 & -1.21424100 & -0.51970100 \\ \mathrm{C} & -3.51158700 & 0.50160800 & 0.27298600 \\ \mathrm{C} & -2.77225700 & 2.73572000 & 0.84546600 \\ \mathrm{H} & -0.67891400 & 3.11732600 & 0.68020900 \\ \mathrm{C} & -3.02026700 & -2.10185300 & -0.63510900 \\ \mathrm{H} & -0.98021300 & -1.54570500 & -0.80226800 \\ \mathrm{C} & -4.56927900 & -0.43586300 & 0.14284900 \\ \mathrm{C} & -3.78429400 & 1.81965900 & 0.71672700 \\ \mathrm{H} & -2.97680800 & 3.74792900 & 1.18055900 \\ \mathrm{C} & -4.33442300 & -1.71539100 & -0.29843600 \\ \mathrm{H} & -2.83070000 & -3.10896200 & -0.99612400 \\ \mathrm{H} & -5.57519900 & -0.11406700 & 0.39997400 \\ \mathrm{H} & -4.81094900 & 2.08909900 & 0.95104700 \\ \mathrm{H} & -5.15145300 & -2.42454400 & -0.39406600 \\ \mathrm{C} & 1.02308800 & 0.97774000 & -2.01385100 \\ \mathrm{H} & 0.87508000 & 2.05588800 & -2.16105700 \\ \mathrm{C} & 2.47505900 & 0.61642600 & -2.33242600 \\ \mathrm{H} & 2.67190900 & 0.83544200 & -3.38755100 \\ \mathrm{H} & 3.19548200 & 1.18241200 & -1.73693100 \\ \mathrm{H} & 2.65992600 & -0.45181700 & -2.17144500 \\ \mathrm{C} & 0.05173600 & 0.22744200 & -2.92266700 \\ \mathrm{H} & 0.14821200 & -0.85715800 & -2.80819300 \\ \mathrm{H} & -0.98964900 & 0.50686500 & -2.74532000 \\ \mathrm{H} & 0.28944500 & 0.47223100 & -3.96349800 \\ \mathrm{C} & 1.64583400 & 2.00911800 & 0.77293900 \\ \mathrm{H} & 1.00606400 & 2.19827500 & 1.64333600 \\ \mathrm{C} & 2.96879600 & 1.43891500 & 1.29488100 \\ \mathrm{H} & 3.46690700 & 2.20438000 & 1.90021700 \\ \mathrm{H} & 2.80884700 & 0.55936900 & 1.92656500 \\ \mathrm{H} & 3.64785000 & 1.16140800 & 0.48347700 \\ \mathrm{C} & 1.87947000 & 3.32397100 & 0.01788100 \\ \mathrm{H} & 0.97300900 & 3.74561700 & -0.42473700 \\ \mathrm{H} & 2.27976700 & 4.06174900 & 0.72123400 \\ \mathrm{H} & 2.61576200 & 3.19946600 & -0.78147900\end{array}$

Sum of electronic and zero-point Energies=

$-1098.869627$

Sum of electronic and thermal Free Energies= $-1098.91757$ 